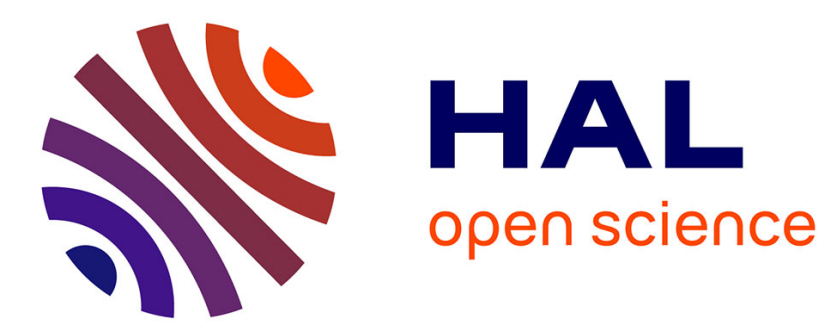

\title{
Thickness control in structural optimization via a level set method
}

Grégoire Allaire, François Jouve, Georgios Michailidis

\section{To cite this version:}

Grégoire Allaire, François Jouve, Georgios Michailidis. Thickness control in structural optimization via a level set method. Structural and Multidisciplinary Optimization, 2016, 53, pp.1349-1382. hal00985000v2

\section{HAL Id: hal-00985000 \\ https://hal.science/hal-00985000v2}

Submitted on 29 Apr 2016

HAL is a multi-disciplinary open access archive for the deposit and dissemination of scientific research documents, whether they are published or not. The documents may come from teaching and research institutions in France or abroad, or from public or private research centers.
L'archive ouverte pluridisciplinaire HAL, est destinée au dépôt et à la diffusion de documents scientifiques de niveau recherche, publiés ou non, émanant des établissements d'enseignement et de recherche français ou étrangers, des laboratoires publics ou privés. 


\title{
Thickness control in structural optimization via a level set method
}

\author{
G. Allaire ${ }^{1}$, F. Jouve ${ }^{2}$, G. Michailidis ${ }^{1,3}$ \\ April 29, 2016 \\ 1 CMAP (UMR CNRS 7641), Ecole Polytechnique, 91128 Palaiseau, France. \\ ${ }^{2}$ University Paris Diderot, Laboratoire J.L. Lions (UMR CNRS 7598), Paris, France. \\ ${ }^{3}$ Renault DREAM-DELT'A Guyancourt, France.
}

\begin{abstract}
In the context of structural optimization via a level-set method we propose a framework to handle geometric constraints related to a notion of local thickness. The local thickness is calculated using the signed distance function to the shape. We formulate global constraints using integral functionals and compute their shape derivatives. We discuss different strategies and possible approximations to handle the geometric constraints. We implement our approach in two and three space dimensions for a model of linearized elasticity. As can be expected, the resulting optimized shapes are strongly dependent on the initial guesses and on the specific treatment of the constraints since, in particular, some topological changes may be prevented by those constraints.
\end{abstract}

Key words: Shape and topology optimization, level-set method, signed distance function, thickness control.

\section{Introduction}

Nowadays there are many efficient shape and topology optimization methods for the design of structures which can handle a large variety of objective functions or mechanical criteria. However, quite often, the resulting optimal shapes are very complex and do not comply with industrial requirements of manufacturability. The way one should post-treat the optimized shape in order to obtain a manufacturable one is not obvious at all and can result in significant modifications and in a final loss of its optimal character. Therefore, it is essential to integrate fabrication constraints in the optimization algorithm in order to ensure an optimal shape which is manufacturable too.

Some of the most significant constraints of this type are related to the thickness of the structure. As a matter of fact, many optimized shapes contain too thin or thick members, or members that are too closely spaced, which must be avoided when standard mass-production fabrication techniques are used. For example, for structures made by casting, thick members should be avoided because of cooling constraints in the manufacturing process while thin members are difficult to fill with liquid metal. Thin members or too closeby members may also violate the precision limitations in the tooling machinery. Beyond manufacturing reasons, imposing a mimimum member size plays a significant role in robust design [43] or mesh independency of the optimal result. It can also be regarded as an implicit way to avoid buckling, without treating explicitly the mechanical constraint. In the same way, limiting the maximum allowed thickness of the structure can be seen as an implicit way to increase the structural redundancy, since the loading path can change and the energy can be redistributed in more members, increasing its robustness, especially in case of loadings under uncertainties [19]. There are many other mechanical reasons for introducing thickness constraints (e.g. optimal design of fluid filters [19]), not to mention those motivations outside from mechanics, such as aesthetics.

In the framework of the homogenization or SIMP method [2], [9] for topology optimization, there is a relatively small body of literature devoted to this issue although many works discussed the notions of filtering or mesh independence. Petersson and Sigmund [36] used a slope constraint for the elements' densities in order to impose a minimum length scale. In [38], Poulsen introduced the so-called "MOLE method" which checks the monotonicity of the density function allong different diagonals of a circle centered at each grid point. Despite its limitations, mentioned in the paper, the MOLE method can also 
be applied in the void part, to avoid small holes in the structure. In [23], Guest et al. proposed to control the minimum length scale by combining projection functions with nodal design variables, defining the density of each node as a weighted sum of the densities of nodes lying at a distance up to the minimum length scale. In [20], Guest applied this method to control simultaneously the minimum length scale of multiple phases. In [19], Guest formulated a constraint of maximum length scale using the volume of balls of diameter equal to this length, centered at each element and in [21] he used a projection scheme in order to optimize the layout of discrete objects having a fixed size and shape and satisfying minimum spacing requirements. The idea of projection functions was also used by Sigmund in [42], while in [43] he proposed to combine projection methods with a formulation for robust design in order to treat manufacturing constraints. Finally, in [51], Zhang et al. imposed a maximum and minimum length scale by setting bounds on the values of the distance function on the skeleton of the shape, constructed via a projected density field.

In the framework of the level-set method [35] for shape and topology optimization [6], [7], [34], [40], [50], there are even fewer papers studying thickness control. In [12] the authors imposed a specific geometric feature and in [11], [28] they added an energy functional in the objective function which favors a family of shapes with strip-like features. The results obtained in these papers show significant changes of the optimal shape compared to the one obtained without adding any geometric constraint. In particular, it seems to give satisfying results for the alleviation of hinges in compliant mechanisms. One of the difficulties in these works is to define in a precise and efficient way the thickness (or other geometric quantities) of a structure, especially since its geometry or shape is implicitly defined by the zero level-set of an auxiliary function. A first attempt for defining the notion of thickness is given in [24].

In the present paper we propose a novel method for handling three major manufacturing constraints of geometric nature using the level-set method for structural optimization: minimal and maximal local thickness and minimal members' distance. They all three rely on a definition of the structure thickness based on the signed distance function of the shape. We then introduce specific integral criteria which exactly measure the satisfaction of each constraint. Our work was previously announced in [5], [29]. Our approach is similar but significantly different from that recently appeared in [24]. We shall compare it, as well as other methods, to our work in Section 9. Of course, it was already noticed by many other authors that the signed distance function is a convenient tool for geometric problems, most notably in image processing (see e.g. [1], [26], [27], [31]). Closely linked to the signed distance function are other important notions that we shall use in the sequel: the "skeleton" or ridge of a shape (which is the location of discontinuities on the distance gradient [1], [26]) and the offset sets of the boundary [1], [26].

The contents of our paper is the following. In Section 2 we recall the optimal design setting as well as basic facts about shape differentiation and level set algorithms. Section 3 is devoted to various classical results on the signed distance function. Section 4 is concerned with the definition of our three geometric constraints, while Section 5 delivers their shape derivatives. In order to handle these constraints in the optimization process we use a simple augmented Lagrangian method, described in Section 6 . It is clear that more efficient optimization algorithms can (and must) be implemented in practice but it is not the goal of the present work to explore this issue. Various important details on the numerical implementation are reported in Section 7 while many numerical results for 2-d and 3-d test cases are exhibited and discussed in Section 8. Many more results can be found in the $\mathrm{PhD}$ thesis of one of the authors [29] (Chapter 3). Eventually Section 9 is an attempt to make comparisons with previous known methods for handling geometric constraints.

\section{Description of the problem}

\subsection{General setting}

Our goal is to optimize a shape $\Omega \subset \mathbb{R}^{d}(d=2$ or 3$)$, a smooth bounded connected domain occupied by a linear isotropic elastic material with Hooke's law $A$ (a positive definite fourth-order tensor). Typically, the boundary of $\Omega$ is comprised of three disjoint parts, such that $\partial \Omega=\Gamma_{D} \cup \Gamma_{N} \cup \Gamma$, with Dirichlet boundary conditions on $\Gamma_{D}$, non-homogeneous Neumann boundary conditions on $\Gamma_{N}$ and homogeneous Neumann boundary conditions on $\Gamma$. For simplicity we assume that only $\Gamma$ is subject to optimization and that $\Gamma_{D}$ and $\Gamma_{N}$ are fixed. We introduce a working domain $D$ (a bounded domain of $\mathbb{R}^{d}$ ) which contains all admissible shapes, that is $\Omega \subset D$. The volume and surface loads are given as two vector-valued functions defined on $D, f \in L^{2}(D)^{d}$ and $g \in H^{1}(D)^{d}$. The displacement field $u$ is the unique solution in 
$H^{1}(\Omega)^{d}$ of the linearized elasticity system

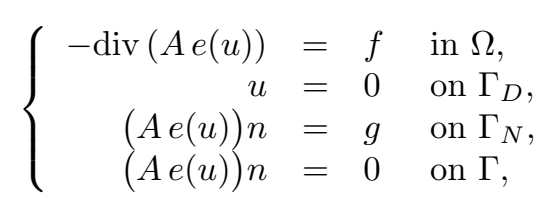

where $e(u)$ is the strain tensor, equal to the symmetrized gradient of $u$. There are many possible choices for the objective function $J(\Omega)$ to be minimized. Here, for simplicity and without loss of generality (since the goal of this study is to deal with geometric constraints), we consider the compliance, or work done by the loads, which reads

$$
J(\Omega)=\int_{\Omega} f \cdot u d x+\int_{\Gamma_{N}} g \cdot u d s=\int_{\Omega} A e(u) \cdot e(u) d x .
$$

A typical shape optimization problem is

$$
\inf _{\Omega \in \mathcal{U}_{a d}} J(\Omega),
$$

where $\mathcal{U}_{a d}$ is the set of admissible shapes. Imposing that all shapes belong to the working domain $D$, that only the free boundary $\Gamma$ is optimizable and that they satisfy a volume constraint $0<V<|D|$, a possible choice of admissible set is

$$
\mathcal{U}_{a d}=\left\{\Omega \subset D \text { such that } \Gamma_{D} \cup \Gamma_{N} \subset \partial \Omega \text { and }|\Omega|=V\right\} .
$$

We shall not dwell on existence issues for optimal shapes. As is well-known [3], [9], problem (3) may lack an optimal solution. Numerically, the non-existence of a minimizer of (3) is reflected by the fact that approximate numerical solutions are mesh dependent (the finer the mesh the more details or finer members in the solution). Classically, to obtain existence of optimal shapes, one needs to restrict further the admissible set $\mathcal{U}_{a d}$ by imposing additional geometrical, topological or smoothness constraints [3], [45], [45], [47]. The addition of geometric constraints related to the thickness is one possible way of restricting the set $\mathcal{U}_{a d}$ and thus deducing existence of optimal shapes (see [14], Section 4.2.5).

\subsection{Shape derivative}

In order to find a descent direction for advecting the shape, we compute a shape derivative for problem (3). The notion of shape derivative dates back, at least, to Hadamard and there has been many contributions to its development (see [37], [47]). In this work, we follow the approach of Murat and Simon for shape derivation [45]. Starting from a smooth reference open set $\Omega$, we consider domains of the type

$$
\Omega_{\theta}=(I d+\theta) \Omega
$$

with $\theta \in W^{1, \infty}\left(\mathbb{R}^{d}, \mathbb{R}^{d}\right)$. It is well known that, for sufficiently small $\theta,(I d+\theta)$ is a diffeomorphism in $\mathbb{R}^{d}$.

Definition 2.1. The shape derivative of $J(\Omega)$ at $\Omega$ is defined as the Fréchet derivative in $W^{1, \infty}\left(\mathbb{R}^{d}, \mathbb{R}^{d}\right)$ at 0 of the application $\theta \rightarrow J((I d+\theta) \Omega)$, i.e.

$$
J((I d+\theta) \Omega)=J(\Omega)+J^{\prime}(\Omega)(\theta)+o(\theta) \quad \text { with } \quad \lim _{\theta \rightarrow 0} \frac{|o(\theta)|}{\|\theta\|}=0
$$

where $\theta \rightarrow J^{\prime}(\Omega)(\theta)$ is a continuous linear form on $W^{1, \infty}\left(\mathbb{R}^{d}, \mathbb{R}^{d}\right)$.

A classical result (Hadamard's structure theorem) states that the shape derivative $J^{\prime}(\Omega)(\theta)$ depends only on the normal trace $\theta \cdot n$ on the boundary $\partial \Omega$. For example, it is known (see e.g. [7]) that, if only the free boundary $\Gamma$ is allowed to vary, the shape derivative of the compliance (2) reads

$$
J^{\prime}(\Omega)(\theta)=-\int_{\Gamma} \theta(s) \cdot n(s) A e(u) \cdot e(u) d s .
$$

In fact, for a great variety of functionals, the shape derivative can be written in the form

$$
J^{\prime}(\Omega)(\theta)=\int_{\partial \Omega} \theta(s) \cdot n(s) V(s) d s,
$$


where the integrand $V$ depends on the specific objective function and boundary conditions. Then, a descent direction can be found by advecting the shape in the direction $\theta(s)=-t V(s) n(s)$ for a small enough descent step $t>0$. For the new shape $\Omega_{t}=(\operatorname{Id}+t \theta) \Omega$, if $V \neq 0$, we can formally write

$$
J\left(\Omega_{t}\right)=J(\Omega)-t \int_{\partial \Omega} V(s)^{2} d s+\mathcal{O}\left(t^{2}\right)<J(\Omega),
$$

which guarantees a descent direction.

In the sequel we shall use sometimes a weaker notion of Gâteaux differentiability.

Definition 2.2. The application $\theta \rightarrow J((I d+\theta) \Omega)$, from $W^{1, \infty}\left(\mathbb{R}^{d}, \mathbb{R}^{d}\right)$ into $\mathbb{R}$, is differentiable in the sense of Gâteaux at 0 if, for any $\theta \in W^{1, \infty}\left(\mathbb{R}^{d}, \mathbb{R}^{d}\right)$, it admits a directional derivative, defined as the following limit (if it exists)

$$
J^{\prime}(\Omega)(\theta)=\lim _{\delta \rightarrow 0} \frac{J((I d+\delta \theta) \Omega)-J(\Omega)}{\delta}
$$

and $\theta \rightarrow J^{\prime}(\Omega)(\theta)$ is a continuous linear form over $W^{1, \infty}\left(\mathbb{R}^{d}, \mathbb{R}^{d}\right)$. Again, $J^{\prime}(\Omega)$ is called the (Gâteaux) shape derivative of $J$.

\subsection{Shape representation in the level set framework}

We favor an Eulerian approach and use the level-set method [35] to capture the shape $\Omega$ on a fixed mesh. Then, the boundary of $\Omega$ is defined by means of a level set function $\psi$ such that

$$
\left\{\begin{aligned}
\psi(x)=0 & \Leftrightarrow x \in \partial \Omega \cap D \\
\psi(x)<0 & \Leftrightarrow x \in \Omega \\
\psi(x)>0 & \Leftrightarrow x \in(D \backslash \bar{\Omega}) .
\end{aligned}\right.
$$

During the optimization process the shape is advected with a velocity $V(x)$ obtained from shape differentiation, as we will see in the sequel. The advection is described in the level-set framework by introducing a pseudo-time $t \in \mathbb{R}^{+}$and solving the well-known Hamilton-Jacobi equation

$$
\frac{\partial \psi}{\partial t}+V|\nabla \psi|=0
$$

using an explicit second order upwind scheme [33], [39].

\subsection{Ersatz material}

Using the so-called "ersatz material" approach, we extend the elasticity equations to the whole domain $D$. To do so, we fill the holes $D \backslash \Omega$ by a weak phase that mimicks the void, but at the same time avoids the singularity of the rigidity matrix. More precisely, we define an elasticity tensor $A^{*}(x)$ which equal to $A$ in $\Omega$ and to $10^{-3} \times A$ in $D \backslash \Omega$ (see [7] for details). Decomposing the boundary $\partial D$ of the working domain in three parts $\partial D=\partial D_{D} \cup \partial D_{N} \cup \partial D_{0}$, such that $\Gamma_{D} \subset \partial D_{D}$ and $\Gamma_{N} \subset \partial D_{N}$, the displacement $u$ is finally computed as the solution of

$$
\left\{\begin{aligned}
-\operatorname{div}\left(A^{*} e(u)\right) & =f & & \text { in } D \\
u & =0 & & \text { on } \partial D_{D} \\
\left(A^{*} e(u)\right) n & =g & & \text { on } \partial D_{N} \\
\left(A^{*} e(u)\right) n & =0 & & \text { on } \partial D_{0} .
\end{aligned}\right.
$$

\section{Signed distance function}

\subsection{Definitions}

The usual Euclidean distance of a point $x \in \mathbb{R}^{d}$ to a closed set $K \subset \mathbb{R}^{d}$ is defined as

$$
d(x, K)=\min _{y \in K}|x-y|
$$

where $|x-y|$ denotes the Euclidean distance between $x$ and $y$. 
Definition 3.1. The signed distance function to a domain $\Omega \subset \mathbb{R}^{d}$ is the function $\mathbb{R}^{d} \ni x \mapsto d_{\Omega}(x)$ defined by:

$$
d_{\Omega}(x)= \begin{cases}-d(x, \partial \Omega) & \text { if } x \in \Omega, \\ 0 & \text { if } x \in \partial \Omega, \\ d(x, \partial \Omega) & \text { if } x \in \bar{c} \Omega\end{cases}
$$

The signed distance function $d_{\Omega}$ must not be confused with the level set function $\psi$ which characterizes the shape $\Omega$ and is the solution of the Hamilton-Jacobi equation (6). Even if the the level set function $\psi$ is frequently reinitialized so that it sastifies $|\nabla \psi|=1$ everywhere in $D$ (which is a desired property for numerical purposes, see e.g. [7]), it is usually not equal to $d_{\Omega}$ since we do not enforce $\psi=0$ on the outer boundary $\partial \Omega \cap \partial D$. We shall come back to this point in Section 7 .

As already said, the signed distance function is convenient to obtain geometric information on the shape $\Omega$. In this view, the notion of skeleton plays an important role in the definition of the thickness of a domain $\Omega$.

Definition 3.2. The skeleton (or ridge) of $\Omega$, denoted $\Sigma$, is the set of points $x \in \mathbb{R}^{d}$ such that the minimum in

$$
d(x, \partial \Omega)=\min _{y \in \partial \Omega}|x-y|
$$

is achieved by at least two distinct points $y$ of $\partial \Omega$.

There are other equivalent definitions or characterizations of the skeleton which help to better understand its structure [31]. First, if one consider a model of propagation for the boundary with a constant velocity along its normal direction, then the skeleton is the location of shocks between "waves" coming from different parts of the boundary. Second, the skeleton is the set of "ridges" (or slope discontinuity) of the signed distance function of the shape. Third, the skeleton is the geometric location of centers of maximal balls, i.e. balls contained in the shape, for which there is no other disk lying inside the shape that contains it (this is Huygens principle, see Figure 1).

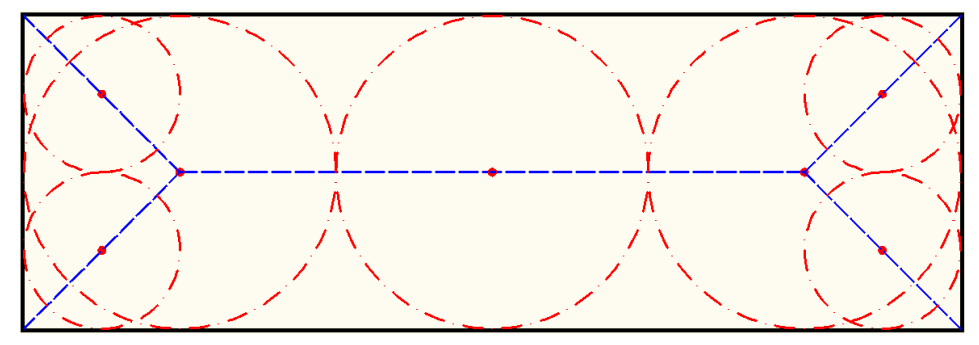

Figure 1: Skeleton (in blue) of a rectangle and maximal balls (in red).

Definition 3.3. The line segment connecting a point $x \in \partial \Omega$ to the skeleton, in the direction of the normal $n(x)$, is called the ray emerging from $x$ and is denoted by ray $(x)$ (see Figure 2).

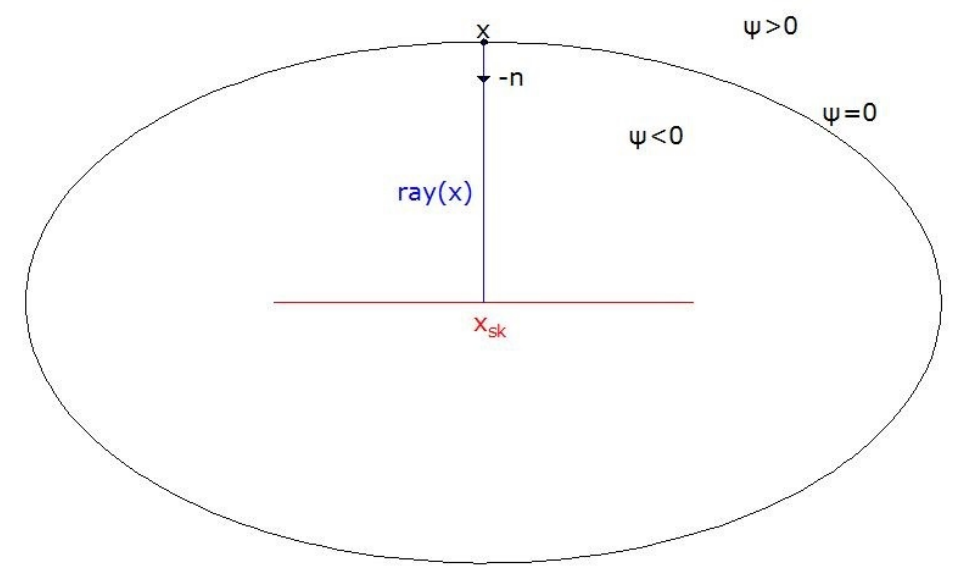

Figure 2: Ray emerging from a point $x \in \partial \Omega$, up to its corresponding point on the skeleton $x_{s k}$. 
We now recall a classical result [8] on the differentiability of the signed distance function, in a somewhat loose manner as far as precise smoothness assumptions are concerned.

Theorem 3.1. Let $\Omega \subset \mathbb{R}^{d}$ be a bounded open set with smooth boundary. Then $d_{\Omega}$ is smooth in a tubular neighborhood of $\partial \Omega$. Moreover, for any point $x \in \mathbb{R}^{d}$,

- either $x \in \partial \Omega$, and then $d_{\Omega}$ is differentiable at $x$, with $\nabla d_{\Omega}(x)=n(x)$, the unit normal vector to $\partial \Omega$ at $x$

- or $x \in \mathbb{R}^{d} \backslash \partial \Omega$, then $d_{\Omega}$ is differentiable at $x$ if and only if $x$ belongs to the complementary of the skeleton of $\Omega$. In such a case, there exists a unique point $p_{\partial \Omega}(x) \in \partial \Omega$, called the projection of $x$ onto $\partial \Omega$, such that $d(x, \partial \Omega)=\left|p_{\partial \Omega}(x)-x\right|$, and the gradient of $d_{\Omega}$ at $x$ reads

$$
\nabla d_{\Omega}(x)=\frac{x-p_{\partial \Omega}(x)}{d_{\Omega}(x)}
$$

Theorem 3.1 implies that $\nabla d_{\Omega}$ remains constant along the rays emerging from the boundary (equal to $\left.n \circ p_{\partial \Omega}\right)$. Furthermore, all points $y$ in a ray ray $(x)$ emerging from $x \in \partial \Omega$ have the same projection point $p_{\partial \Omega}(y)=x$.

\subsection{Shape derivative of the signed distance function}

The shape derivation of the signed distance function has been studied in details by Delfour and Zolesio in [17], [16]. For the sake of completeness, we recall this result with a formal but illuminating proof which can also be found in [25]. For a rigorous proof and further details, the interested reader is referred to [4], [17], [16].

Proposition 3.1. Assume $\Omega \subset D$ is an open set of class $\mathcal{C}^{1}$, and fix a point $x \notin \Sigma$. Then $\theta \mapsto d_{(I d+\theta) \Omega}(x)$ is Gâteaux-differentiable at $\theta=0$, as an application from $W^{1, \infty}\left(D, \mathbb{R}^{d}\right)$ into $\mathbb{R}$, and its shape derivative is

$$
d_{\Omega}^{\prime}(\theta)(x)=-\theta\left(p_{\partial \Omega}(x)\right) \cdot n\left(p_{\partial \Omega}(x)\right) .
$$

Proof. We content ourselves with a simple albeit formal proof. The signed distance function can be seen as the unique (viscosity) solution of the Hamilton-Jacobi equation

$$
\begin{cases}\left|\nabla d_{\Omega}(x)\right|=1 & \text { in } D \\ d_{\Omega}(x)=0 & \text { on } \partial \Omega .\end{cases}
$$

The boundary condition can be written in the weak form

$$
\int_{\partial \Omega} d_{\Omega}(x) \phi(x) d x=0 \quad \text { for any smooth test function } \phi .
$$

By the chain rule lemma, its shape derivative is

$$
\int_{\partial \Omega} d_{\Omega}^{\prime}(\theta)(x) \phi(x) d x+\int_{\partial \Omega} \theta(x) \cdot n(x)\left(\frac{\partial\left(d_{\Omega} \phi\right)}{\partial n}(x)+H(x) d_{\Omega}(x) \phi(x)\right) d x=0
$$

where $H$ is the mean curvature. Since $d_{\Omega}$ vanishes on the boundary, $\frac{\partial d_{\Omega}}{\partial n}=\nabla d_{\Omega} \cdot n=1$ and $\phi$ is any test function, we deduce

$$
d_{\Omega}^{\prime}(\theta)(x)=-\theta(x) \cdot n(x), \quad \forall x \in \partial \Omega .
$$

Furthermore, differentiating the eikonal equation $\left|\nabla d_{\Omega}(x)\right|^{2}=1$ yields

$$
2 \nabla d_{\Omega}(x) \cdot \nabla d_{\Omega}^{\prime}(\theta)(x)=0 \text { in } D
$$

which implies that $d_{\Omega}^{\prime}(\theta)(x)$ is constant along the integral curves of the vector field $\nabla d_{\Omega}$. By Theorem 3.1 the integral curves of $\nabla d_{\Omega}$ are the rays (along which $\nabla d_{\Omega}$ remains constant equal to the normal $n$ at the boundary). Therefore, the shape derivative $d_{\Omega}^{\prime}(\theta)(x)$ at a point $x \notin \Sigma$ is equal to its value at the foot of the ray, $d_{\Omega}^{\prime}(\theta)\left(p_{\partial \Omega}(x)\right)$, for which there is the explicit expression (10). 


\section{Formulation of thickness constraints}

The notion of thickness in structural mechanics can be understood in different ways. For example, in a truss composed of beams with circular cross-section, it can be claimed that the thickness of a beam is equal to the diameter of the cross-section. Similar intuitive definitions can be given for a variety of specific structures. However, for general non-parametric structures, things become more complicated, especially if the geometry and topology of its shape vary considerably during the optimization process. Therefore, we give a definition of thickness which is not relying on any particular parametrization or mechanical model and which is able to sustain drastic changes in geometry and topology. This definition is based on the signed distance function introduced in Section 3. It turns out that it is a different definition when maximum or minimum thickness are considered. Although these definitions are satisfying from a mathematical point of view and are very convenient and robust from a numerical point of view, we shall see that they are not immune to some strange geometrical effects.

\subsection{Maximum thickness}

A constraint of maximum thickness of size $d_{\max }$ is interpreted as: there is no point in the shape, which is the center of a ball of diameter $d_{\max }$ fully covered by material. In other words, the largest (absolute) value of the signed distance function in $\Omega$ (which is attained on the skeleton) is smaller than $d_{\max } / 2$. This definition is well suited for the signed distance function to the boundary of the shape.

Definition 4.1. A shape $\Omega$ has thickness smaller than $d_{\max }>0$ if

$$
d_{\Omega}(x) \geq-d_{\max } / 2, \quad \forall x \in \Omega .
$$

(Recall that $d_{\Omega}$ takes negative values inside the domain $\Omega$.)

A possible drawback of the above definition is the appearance of distortions at the regions of crossing between bars. In Figure 3(a), we see two bars of uniform thickness $d_{\max }$ crossing. Although intuitively one would say that the maximum thickness constraint (12) is respected, there is a region around the center of the joint, where the pointwise constraint (12) is violated. One possible solution, satisfying constraint (12) is given in Figure 3(b). However, such distortions of the shape close to the position of joints are not usually preferable for engineers, both for mechanical or manufacturing reasons. In Sections 4.4 and 7 , we shall discuss ways to avoid such distortions.

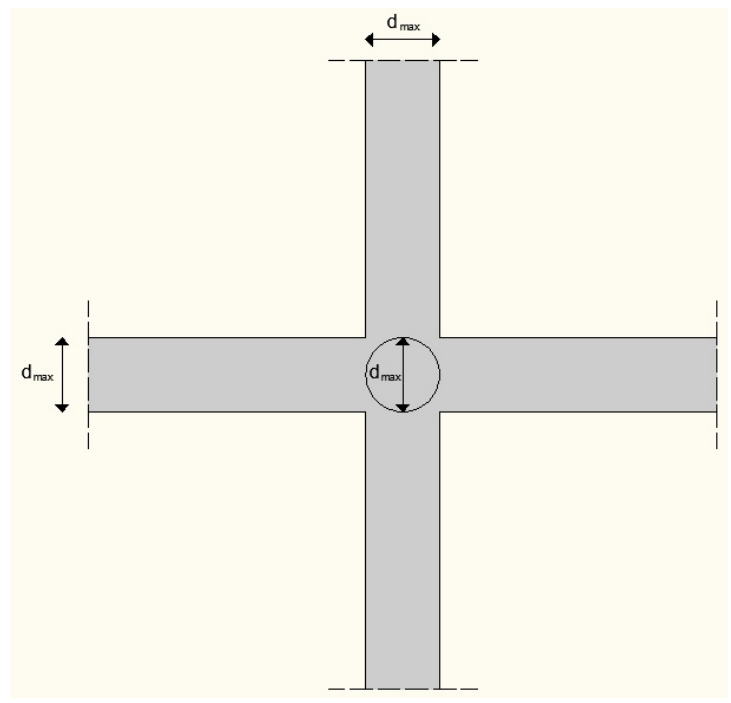

(a)

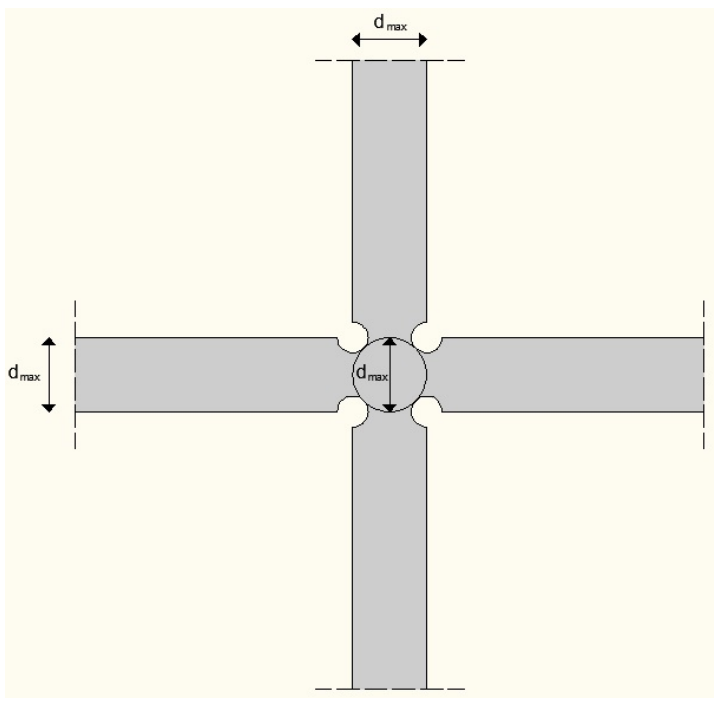

(b)

Figure 3: (a): bars violating the thickness constraint (12), (b): bars respecting the thickness constraint $(12)$.

\subsection{Minimum thickness}

The formulation of this constraint is not as straightforward as the previous one, since it is not obvious how the values of the signed distance function are related to a notion of minimum thickness. However, 
another definition based on offset sets [1], [26] gives an intuitive view of a minimum thickness constraint.

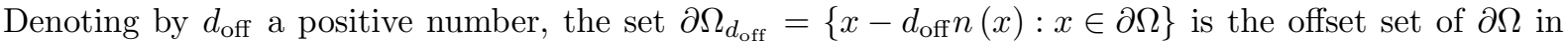
the direction $-n(x)$ at a distance $d_{\text {off }}$ (see Figure 4$)$.

Definition 4.2. A shape $\Omega$ has thickness larger than $d_{\min }>0$ if

$$
d_{\Omega}\left(x-d_{\text {off }} n(x)\right) \leq 0, \quad \forall x \in \partial \Omega, \forall d_{\text {off }} \in\left[0, d_{\text {min }}\right]
$$

In other words, (13) guarantees that any offset set in the direction $-n(x)$ (i.e., directed inside $\Omega$ ) up to a distance $d_{\min }$ stays inside the shape $\Omega$. We emphasize that inequality (13) must be valid for the whole interval $\left[0, d_{m i n}\right]$ of offset sets. A single offset set at the desired distance $d_{m i n}$ cannot detect the case of members that are very thin and are also very close to each other. This case is illustrated on Figure 4 which displays two members (in grey color) of a structure $\Omega$. The offset set for one single value $d_{\text {off }}$ can fall entirely in the structure without detecting the void between the two parts. In this case satisfying (13), restricted to the sole value $d_{\text {off }}$, does not prevent the violation of the constraint.

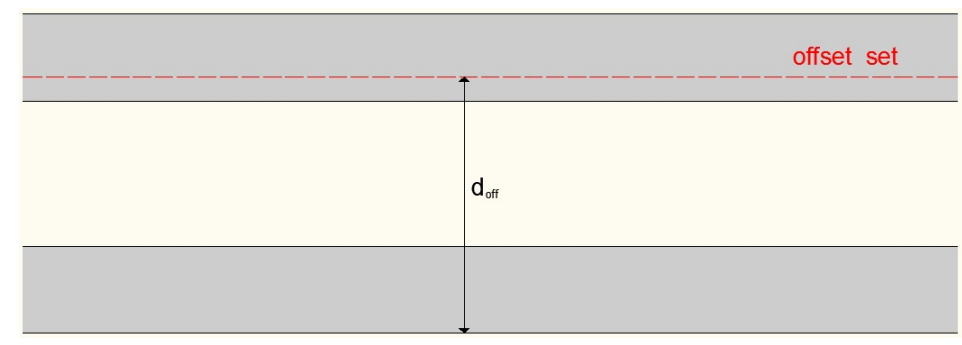

Figure 4: Inability to detect very thin members with a single offset set (shape $\Omega$ in grey).

Remark 4.1. In [24] a different formulation of the minimum thickness constraint is proposed. Instead of using offset sets, it is similar to (12) but restricted to points on the skeleton. We shall discuss this in Subsection 9.2.1 and show that it is not easy to differentiate contrary to our proposed definition (13).

Remark 4.2. Definition 4.2 involves the normal vector at each point of the boundary. Therefore it is implictly assumed that the shape is at least of class $C^{1}$. For sure, it cannot be extended to non-smooth shapes, having for examples cusps. It can be extended to Lipschitz shapes (which may have corners) by enforcing (13) for any normal vector in the subdifferential of the corner (the subdifferential of a Lipschitz function is defined in the sense of Clarke [13]). This is not a practical definition since, on the one hand, it is not easy to define the subdifferential of a given corner and, on the other hand, this constraint would not be shape differentiable.

\subsection{Minimum members' distance}

This constraint states that the distance between two structure members is larger than some minimal value $d_{m m d}>0$. It looks exactly the same as the minimum thickness constraint, except that it is now imposed on the complementary of the shape. We just need to invert the direction of offsetting. In case of members surrounding a hole, this notion of minimum members' distance is equivalent to a minimum size for that hole. However, we prefer not to use this terminology since the minimum members' distance can also apply to a void region which is not a hole but rather the exterior of the shape.

Definition 4.3. A shape $\Omega$ has members' distance larger than $d_{m m d}>0$ if

$$
d_{\Omega}\left(x+d_{\mathrm{off}} n(x)\right) \geq 0, \quad \forall x \in \partial \Omega, \forall d_{\mathrm{off}} \in\left[0, d_{m m d}\right] .
$$

\subsection{Penalty functionals}

Constraints (12), (13) and (14) are pointwise, i.e. they are defined at each point of the domain $\Omega$ for (12) or of its boundary $\partial \Omega$ for (13) and (14). They are infinite in number and thus they cannot be treated numerically as such. If one replaces them by a maximal or minimal value over all points, then they become non-differentiable which is a difficulty too. Even upon discretization of the domain by a mesh and of the boundary by a level set function, the number of points which are constrained is of the order of the number of points in the shape $\Omega$ or on the boundary $\partial \Omega$ : in practice it is a too large number to be tackled efficiently by optimization algorithms. 
For this reason, it is advantageous to formulate global averaged constraints. A simple choice of a smooth global constraint is to use a quadratic penalty function. This function takes the following form for the three previous constraints.

$$
\begin{gathered}
\text { Maximum Thickness: } P_{\text {MaxT }}(\Omega)=\int_{\Omega}\left[\left(d_{\Omega}(x)+d_{\max } / 2\right)^{-}\right]^{2} d x, \\
\text { Minimum Thickness: } P_{M i n T}(\Omega)=\int_{\partial \Omega} \int_{0}^{d_{\text {min }}}\left[\left(d_{\Omega}(s-\xi n(s))\right)^{+}\right]^{2} d \xi d s, \\
\text { Minimum Members' Distance: } P_{M M D}(\Omega)=\int_{\partial \Omega} \int_{0}^{d_{m m d}}\left[\left(d_{\Omega}(s+\xi n(s))\right)^{-}\right]^{2} d \xi d s,
\end{gathered}
$$

with the usual notations $(f)^{+}=\max (f, 0)$ and $(f)^{-}=\min (f, 0)$.

One can check that a pointwise constraint is satisfied if and only if its corresponding penalty functional is equal to zero. For example, for the maximum thickness functional $P_{M a x T}$ :

$$
P_{M a x T}(\Omega)=0 \quad \Leftrightarrow \quad\left(d_{\Omega}(x)+d_{\max } / 2\right)^{-}=0 \quad \forall x \in \Omega \quad \Leftrightarrow \quad d_{\Omega}(x) \geq-d_{\max } / 2 \quad \forall x \in \Omega .
$$

Although the penalty functional (15) for the maximum thickness constraint is convenient from a theoretical point of view, numerically it is not satisfactory for two reasons. First, it is so "strict" that it is prone to create artifacts like the one depicted in Figure 3 for joints at bars crossing (which is indeed good for minimizing (15) but not so desirable from a practical point of view). Thus we would like to loosen somehow this constraint in order to get more regular shapes. Second, when the constraint $P_{\operatorname{MaxT}}(\Omega)=0$ is satisfied there is no information, neither in the function $P_{\operatorname{Max} T}(\Omega)$, nor in its shape gradient, about how much margin is available in the satisfaction of the constraint. Therefore, we want to change (15) into an inequality constraint which would indicate if it is largely or marginally satisfied. We suggest the following "regularized" formulation that we use for our numerical examples

$$
P_{M a x T}(\Omega)=\left(\frac{\int_{\Omega} f\left(d_{\Omega}(x)\right) d_{\Omega}(x)^{2} d x}{\int_{\Omega} f\left(d_{\Omega}(x)\right) d x}\right)^{\frac{1}{2}} \leq d_{\max } / 2,
$$

where $f$ is a regularization function

$$
f\left(d_{\Omega}(x)\right)=0.5\left(1+\tanh \left(\frac{\left|d_{\Omega}(x)\right|-d_{\max } / 2}{\alpha_{f} d_{\max } / 2}\right)\right),
$$

$\alpha_{f}>0$ being a parameter that controls the regularization of the constraint. If (18) is satisfied, it may happen that (12) is violated at some points in $\Omega$, or equivalently that (15) does not vanish. Nevertheless, (12) is satisfied in an averaged sense and, more importantly, the smaller the left hand side of (18) is, the better the constraint is satisfied. This approximation is consistent in the sense that, when $\alpha_{f}$ goes to $0, f\left(d_{\Omega}\right)$ converges to $H\left(\left|d_{\Omega}\right|-d_{\max } / 2\right)$, the Heaviside function at point $\left(d_{\max } / 2\right)$ (see Figure 5 ), and satisfying inequality (18) is equivalent to have (15) equal to 0 . In this work, we have set $a_{f}=\frac{4 \Delta x}{5 d_{\max }}$, where $\Delta x$ is the uniform mesh size.

Remark 4.3. One may wonder if such a regularization process could be applied to the minimum thickness constraint (13) or to the minimum members' distance constraint (14). Unfortunately, it does not seem to work in these latter cases. Indeed, contrary to the maximum thickness constraint (12), the other constraints (13) and (14) do not provide any information about the extent of violation or satisfaction. Therefore, their reformulation in a global form of the same type as (18) is not possible.

Eventually, we replace the unconstrained optimization problem (3) by the following one

$$
\begin{array}{rl}
\inf _{\Omega \in \mathcal{U}_{a d}} & J(\Omega) \\
\text { such that } & P_{\operatorname{MinT}}(\Omega)=0 \\
& P_{M M D}(\Omega)=0 \\
& P_{\operatorname{MaxT}}(\Omega) \leq d_{\max } / 2
\end{array}
$$

or any variant with a smaller number of constraints. In $(19)$ the constraint $P_{M a x T}(\Omega)$ is computed by formula (18). 


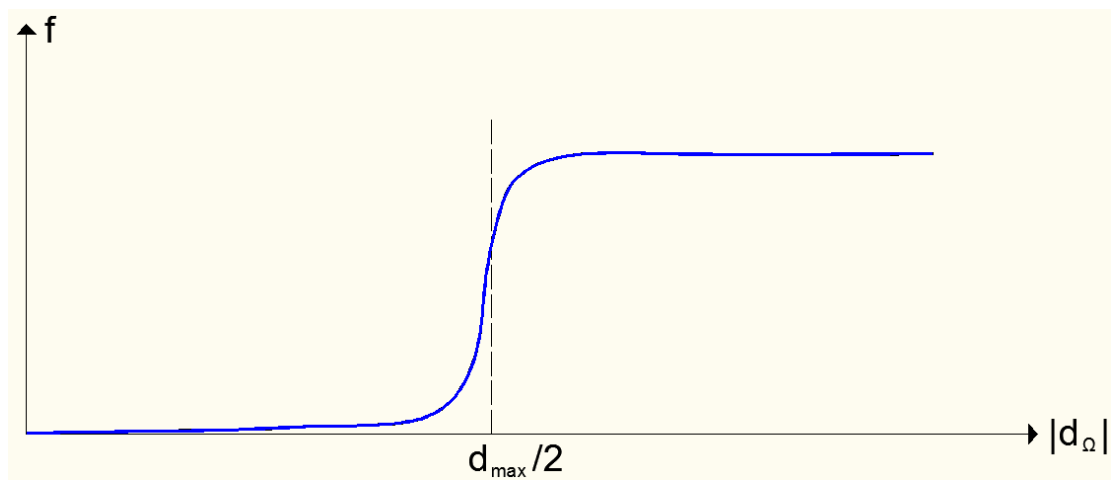

(a)

Figure 5: Graph of the regularization function $f\left(d_{\Omega}(x)\right)$.

Remark 4.4. The minimum thickness constraint yields an existence result of optimal shapes for the constrained optimization problem (19). Indeed, when the constraint (13) is satisfied, it implies that the shape $\Omega$ has some additional regularity. Not only is the normal $n$ well defined everywhere on the boundary $\partial \Omega$ but it has some uniform regularity since there exists a tubular neighborhood of $\partial \Omega$ inside $\Omega$. The minimal thickness prevents the onset of too many small holes in a minimizing sequence for (19). Such a property has been the starting point for a proof of existence of optimal designs in [14] (see Section 4.2.5).

\subsection{Discussion on topological changes}

As we shall soon see, the constraints (15), (16) and (17) are well defined in a geometric shape optimization framework, in the sense that we can compute their shape derivatives and thus apply standard gradientbased optimization algorithms. However, it turns out that these constraints are not continuous with respect to topological changes and are thus very sensitive to topological changes. In particular, they don't admit a topological derivative. The reason is that the signed distance function $d_{\Omega}$ is not continuous with respect to $\Omega$ upon insertion of a small hole.

This observation can be validated numerically, through the existence of very small holes, the absence of which would result in a severe violation of a maximum thickness constraint. The appearance of such holes can have severe numerical implications for our method, making the optimization algorithm quite unstable. If one wants to avoid such tiny holes, one should impose at the same time a constraint on their minimal size. Else, it is inevitable that such holes can appear in the optimized shape, since they are usually preferable for solving an optimization problem under a maximum thickness constraint.

Let us give an example in order to better understand this problem. Consider two bars crossing at 90 degrees, like in Figure 3, with a small hole in the middle of the joint. The bars have a uniform thickness of 0.2. Setting a constraint of maximum thickness with $d_{\max }=0.2$, we can see the great difference caused by the existence of a small hole in Figure 6. The existence of the hole reduces significantly the values of $d_{\Omega}$ and as a result no violation of the thickness appears. On the contrary, the elimination of the hole changes dramatically the values of $d_{\Omega}$ and a region around the center of the joint appears, where the thickness constraint is violated.

There are two mechanisms for the appearance of such holes. The first one, which is the most usual, is the reduction in size of an existing hole. The second one is more tricky and consists in splitting an existing hole into two holes (see [29], pp. 82, Figure 3.24).

Another consequence of the huge impact of tiny holes on the values of $d_{\Omega}$ and consequently on those of $P_{\text {MaxT }}(\Omega)$ is the non existence of optimal solutions to problem (3) under a maximum thickness constraint, in the absence of further geometrical restrictions. Indeed, for compliance minimization, small perforations have an infinitesimal impact on the compliance while they dramatically reduce the maximal thickness, allowing constraint (15) to be satisfied. Therefore, any maximal thickness $d_{\max }$ will do, by introducing sufficiently enough small holes, and the minimal compliance would be asymptotically approached (but not realized) by holes sizes going to zero. The next paragraph gives one possible remedy to this inconvenient. 


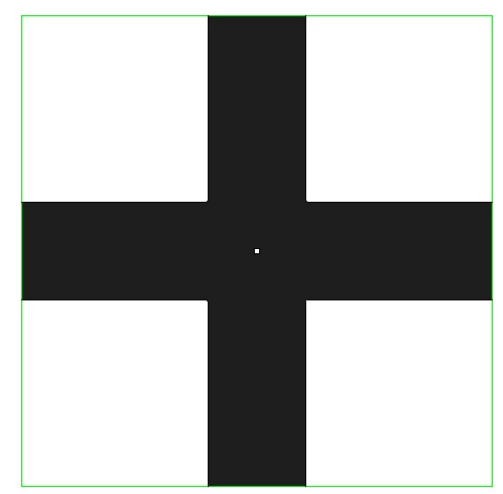

(a)

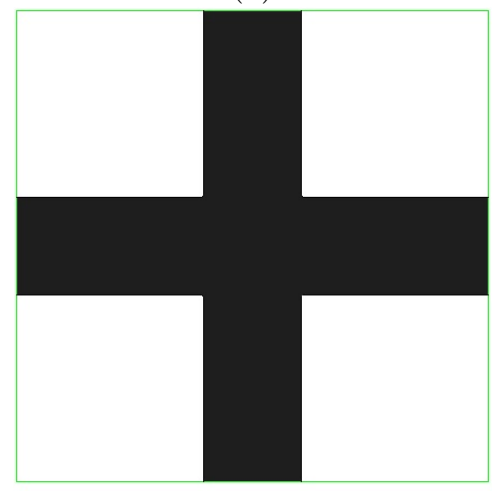

(d)

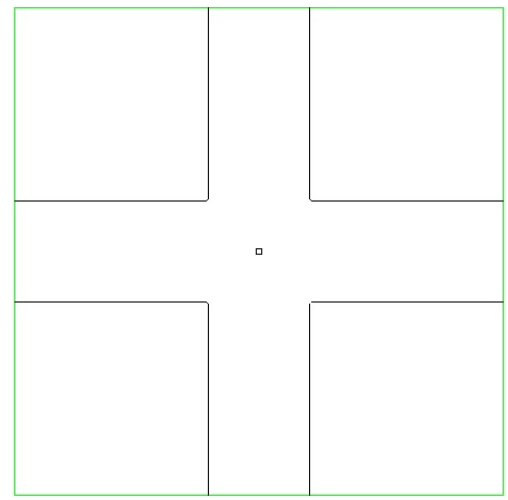

(b)

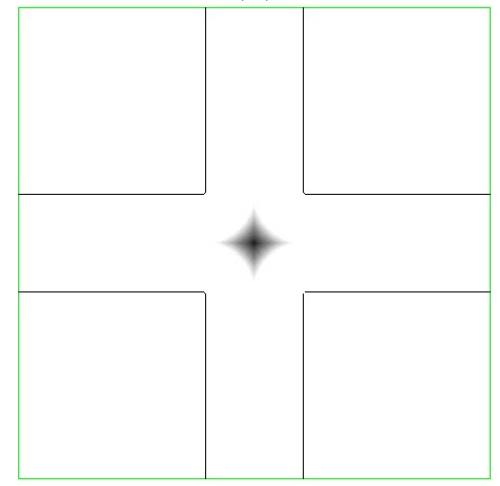

(e)

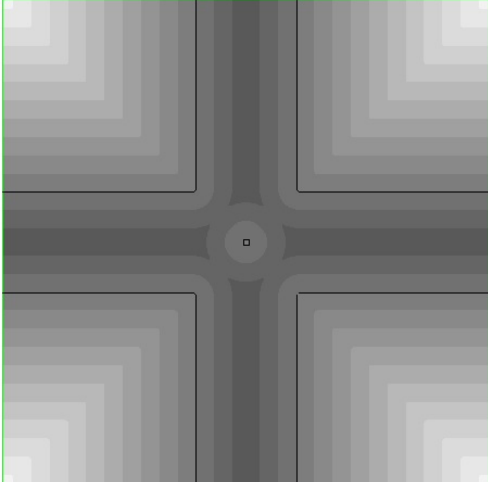

(c)

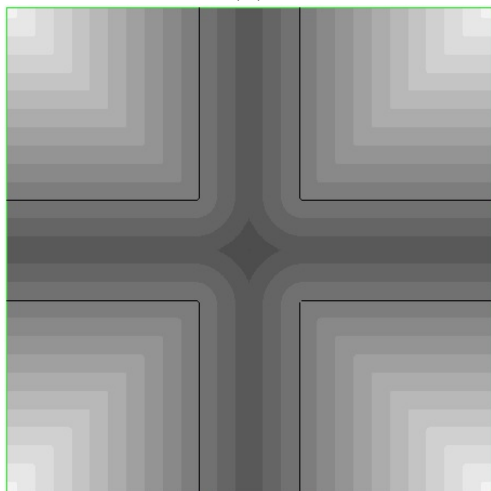

(f)

Figure 6: Joint with (upper row) and without (lower row) a hole in the middle; (a),(d): shape in black $(\Omega)$; (b),(e): thickness violation; (c),(f): $2 \mathrm{~d}$ plot of $d_{\Omega}$.

\section{Shape derivative of penalty functionals}

This section is devoted to the computation of shape derivatives of the penalty functionals defined in Section 4.4. We always assume that the shape is at least of class $C^{2}$ since we use a co-aera formula which involves the principal curvatures of its boundary.

\subsection{Maximum thickness}

Lemma 5.1. The shape derivative of (15) is

$$
P_{M a x T}^{\prime}(\Omega)(\theta)=\int_{\partial \Omega}-\theta(x) \cdot n(x) \int_{\operatorname{ray}(x) \cap \Omega} 2\left(d_{\Omega}(z)+d_{\max } / 2\right)^{-} \prod_{i=1}^{d-1}\left(1+d_{\Omega}(z) \kappa_{i}(x)\right) d z d x,
$$

where $\kappa_{i}(x)$ are the principal curvatures at the point $x \in \partial \Omega$.

Remark 5.1. The shape derivation of the regularized version (18) of the maximum thickness constraint follows exactly the same steps described in the following proof.

Proof. The shape derivative of (15) reads

$$
P_{\text {MaxT }}^{\prime}(\Omega)(\theta)=\int_{\Omega} 2 d_{\Omega}^{\prime}(x)(\theta)\left(d_{\Omega}(x)+d_{\max } / 2\right)^{-} d x .
$$

Using a co-area formula [4], [18], we can decompose the volume integral into a surface integral along the boundary of the shape and an integral along the rays emerging from it. Then, the shape derivative (21) takes the following form

$$
P_{\text {MaxT }}^{\prime}(\Omega)(\theta)=\int_{\partial \Omega} \int_{\operatorname{ray}(x) \cap \Omega} 2 d_{\Omega}^{\prime}(z)(\theta)\left(d_{\Omega}(z)+d_{\max } / 2\right)^{-} \prod_{i=1}^{d-1}\left(1+d_{\Omega}(z) \kappa_{i}(x)\right) d z d x .
$$


Since $d_{\Omega}^{\prime}(\theta)$ is constant along the ray emerging from $x$, it can be moved out of the second integral:

$$
P_{\text {MaxT }}^{\prime}(\Omega)(\theta)=\int_{\partial \Omega} d_{\Omega}^{\prime}(x)(\theta) \int_{\operatorname{ray}(x) \cap \Omega} 2\left(d_{\Omega}(z)+d_{\max } / 2\right)^{-} \prod_{i=1}^{d-1}\left(1+d_{\Omega}(z) \kappa_{i}(x)\right) d z d x
$$

and since its expression on the boundary is known (see equation (10)), we finally end up with

$$
P_{M a x T}^{\prime}(\Omega)(\theta)=\int_{\partial \Omega}-\theta(x) \cdot n(x) \int_{\operatorname{ray}(x) \cap \Omega} 2\left(d_{\Omega}(z)+d_{\max } / 2\right)^{-} \prod_{i=1}^{d-1}\left(1+d_{\Omega}(z) \kappa_{i}(x)\right) d z d x,
$$

which completes the proof.

\subsection{Minimum thickness}

To make the text more compact, we change the notation according to Figure 7 . We denote by $x_{m}=$ $x-\xi n(x)$ the offset point, from a point $x \in \partial \Omega$, at a distance $\xi$ in the direction opposite to the normal vector, and $p_{\partial \Omega}\left(x_{m}\right)$ the projection of $x_{m}$ on the boundary. For small values of $\xi$ (such that $x_{m}$ has not crossed the skeleton), the projection $p_{\partial \Omega}\left(x_{m}\right)$ is simply equal to $x$. Once $x_{m}$ has crossed the skeleton inside $\Omega, p_{\partial \Omega}\left(x_{m}\right)$ differs from $x$.

Lemma 5.2. The shape derivative of (16) is

$$
\begin{aligned}
& P_{\text {Min } T}^{\prime}(\Omega)(\theta)= \\
& \int_{\partial \Omega} \int_{0}^{d_{\min }} \theta(x) \cdot n(x)\left[H(x)\left(\left(d_{\Omega}\left(x_{m}\right)\right)^{+}\right)^{2}+2\left(d_{\Omega}\left(x_{m}\right)\right)^{+} \nabla d_{\Omega}\left(x_{m}\right) \cdot \nabla d_{\Omega}(x)\right] d \xi d x \\
& -\int_{\partial \Omega} \int_{0}^{d_{\min }} \theta\left(p_{\partial \Omega}\left(x_{m}\right)\right) \cdot n\left(p_{\partial \Omega}\left(x_{m}\right)\right) 2\left(d_{\Omega}\left(x_{m}\right)\right)^{+} d \xi d x,
\end{aligned}
$$

where $H(x)$ denotes the mean curvature at the point $x \in \partial \Omega$.

Remark 5.2. The same analysis holds for the quadratic penalty function (17) used in the Minimum Members' Distance constraint: we do not repeat the arguments here.

Proof. Using a classical chain-rule lemma for the shape derivative of surface integrals with shape-dependent integrands [3], the shape derivative of (16) reads

$$
\begin{aligned}
& P_{\text {MinT }}^{\prime}(\Omega)(\theta)= \\
& \left.\int_{\partial \Omega} \int_{0}^{d_{\min }} \theta(x) \cdot n(x)\right)\left[H(x)\left(\left(d_{\Omega}\left(x_{m}\right)\right)^{+}\right)^{2}+\frac{\partial}{\partial n}\left(\left(d_{\Omega}\left(x_{m}\right)\right)^{+}\right)^{2}\right] d \xi d x \\
& +\int_{\partial \Omega} \int_{0}^{d_{\text {min }}} \frac{\partial}{\partial \Omega}\left(\left(d_{\Omega}\left(x_{m}\right)\right)^{+}\right)^{2}(\theta) d \xi d x \\
& \left.=\int_{\partial \Omega} \int_{0}^{d_{\min }} \theta(x) \cdot n(x)\right)\left[H(x)\left(\left(d_{\Omega}\left(x_{m}\right)\right)^{+}\right)^{2}+2\left(d_{\Omega}\left(x_{m}\right)\right)^{+} \nabla d_{\Omega}\left(x_{m}\right) \cdot n(x)\right] d \xi d x \\
& +\int_{\partial \Omega} \int_{0}^{d_{\text {min }}} 2\left(d_{\Omega}\left(x_{m}\right)\right)^{+} d_{\Omega}^{\prime}\left(x_{m}\right)(\theta) d \xi d x .
\end{aligned}
$$

¿From equation (11), we know that the shape derivative of the signed distance function at the offset point $x_{m}$ will be equal to that of its projection point on the boundary $p_{\partial \Omega}\left(x_{m}\right)$, for which we can use an explicit formula

$$
d_{\Omega}^{\prime}\left(x_{m}\right)(\theta)=d_{\Omega}^{\prime}\left(p_{\partial \Omega}\left(x_{m}\right)\right)(\theta)=-\theta\left(p_{\partial \Omega}\left(x_{m}\right)\right) \cdot n\left(p_{\partial \Omega}\left(x_{m}\right)\right) .
$$

Using the fact that $n(x)=\nabla d_{\Omega}(x)$, for any $x \in \partial \Omega$, and substituting equation (24) in (23) yields the desired result. 


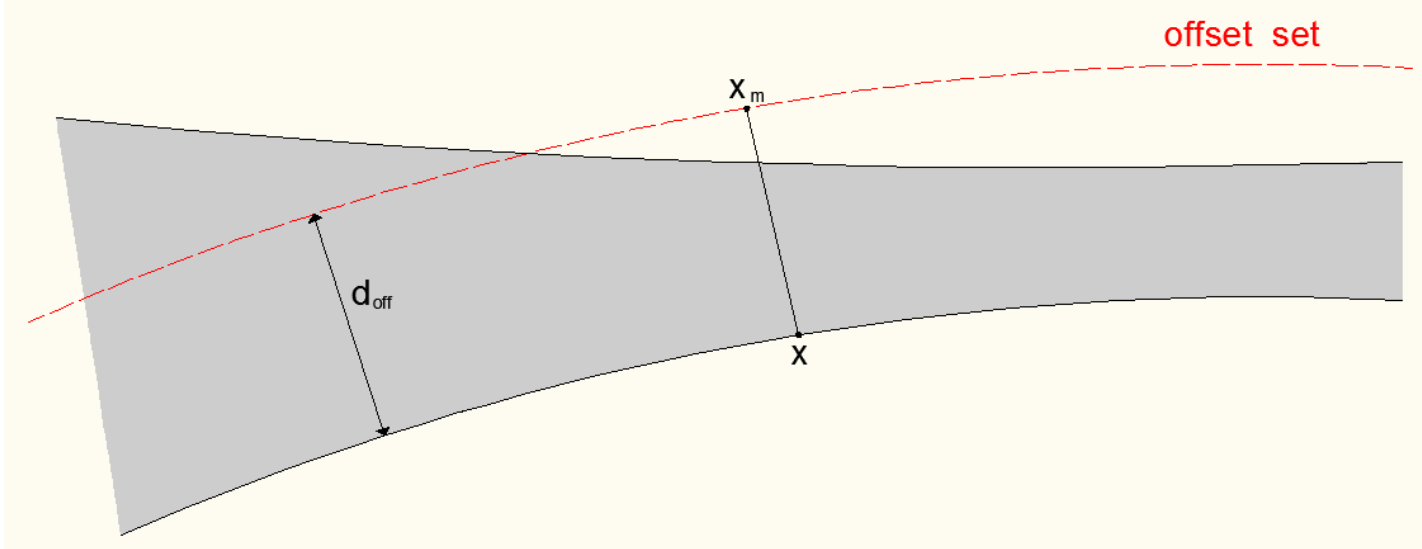

Figure 7: Offset point $x_{m}=x-d_{\text {off }} n(x)$.

\section{Augmented Lagrangian method and descent direction}

In this section we describe a simple augmented Lagrangian algorithm for minimizing a cost function $J(\Omega)$ under equality constraints

$$
P_{i}(\Omega)=0, \quad i=1, \ldots, m .
$$

Of course, there are other more efficient optimization algorithms in topology optimization, e.g. MMA [48] or MFD [30], but it is not the purpose of the present paper to discuss the important issue of optimization efficiency. Rather we focus on the introduction of the thickness constraints of Section 4.4 in the optimization problem (3).

Following [32] we introduce the augmented Lagrangian function

$$
L(\Omega, \ell, \mu)=J(\Omega)-\sum_{i=1}^{m} \ell_{i} P_{i}(\Omega)+\sum_{i=1}^{m} \frac{\mu_{i}}{2} P_{i}^{2}(\Omega),
$$

where $J(\Omega)$ is the cost function, $\ell=\left(\ell_{i}\right)_{i=1, \ldots, m}$ the Lagrange multipliers and $\mu=\left(\mu_{i}\right)_{i=1, \ldots, m}$ the penalty parameters, used to enforce the constraints at convergence. The Lagrange multipliers are updated at each iteration $n$ according to the relation $\ell_{i}^{n+1}=\ell_{i}^{n}-\mu_{i} P_{i}\left(\Omega_{n}\right)$ (see [32] for more details). We also increase the penalty parameters every 5 iterations. A similar approach is followed for the case of inequality constraints (see [32]).

For example, for the optimization problem

$$
\begin{array}{rl}
\min _{\Omega \in \mathcal{U}_{a d}} & J(\Omega)=\int_{\Omega} f \cdot u d x+\int_{\Gamma_{N}} g \cdot u d s, \\
\text { s.t. } & P_{1}(\Omega)=\int_{\Omega} d x-\alpha_{V}|D|=0,\left(0<\alpha_{V}<1\right), \\
& P_{2}(\Omega)=P_{M a x T}(\Omega)=\int_{\Omega}\left[\left(d_{\Omega}(x)+d_{\max } / 2\right)^{-}\right]^{2} d x=0,
\end{array}
$$

where $u$ is the solution of (7), we construct the augmented Lagrangian function

$$
L(\Omega, \ell, \mu)=\int_{\Omega} f \cdot u d x+\int_{\Gamma_{N}} g \cdot u d s-\sum_{i=1}^{2} \ell_{i} P_{i}(\Omega)(\theta)+\sum_{i=1}^{2} \frac{\mu_{i}}{2} P_{i}^{2}(\Omega),
$$

which can now be regarded as the new objective function to minimize. Building on the results of the previous sections, its shape derivative is

$$
L^{\prime}(\Omega, \ell, \mu)(\theta)=-\int_{\Gamma} \theta(x) \cdot n(x) A e(u) \cdot e(u) d x-\sum_{i=1}^{2} \ell_{i} P_{i}^{\prime}(\Omega)(\theta)+\sum_{i=1}^{2} \mu_{i} P_{i}(\Omega) P_{i}^{\prime}(\Omega)(\theta),
$$

where $\Gamma$ is the optimizable part of the boundary $\left(\Gamma_{D}\right.$ and $\Gamma_{N}$ are kept fixed),

$$
P_{1}^{\prime}(\Omega)(\theta)=\int_{\Gamma} \theta(x) \cdot n(x) d x \quad \text { and } \quad P_{2}^{\prime}(\Omega)(\theta)=-\int_{\Gamma} \theta(x) \cdot n(x) g(x) d x,
$$


with

$$
g(x)=\int_{\operatorname{ray}(x) \cap \Omega} 2\left(d_{\Omega}(z)+d_{\max } / 2\right)^{-} \prod_{i=1}^{d-1}\left(1+d_{\Omega}(z) \kappa_{i}(x)\right) d z .
$$

A descent direction is revealed as

$$
\theta(x)=-n(x)\left(-A e(u) \cdot e(u)-\ell_{1}+\mu_{1} P_{1}(\Omega)+\ell_{2} g(x)-\mu_{2} P_{2}(\Omega) g(x)\right) .
$$

The descent direction is of the type $V(x) n(x)$ and the normal velocity $V$ can be used in the HamiltonJacobi equation (6). A priori formula (28) makes sense only at the noundary $\partial \Omega$. Therefore, it must be extended to the whole computational domain $D$ since the Hamilton-Jacobi equation (6) is solved everywhere in $D$. As explained in [7], [15] it is possible (and numerically efficient) to extend the shape derivative and regularize it at the same time. More precisely, restricting our attention to descent directions of the type $\theta=Q n$, we solve the following extension-regularization problem: find the solution $Q \in H^{1}(D)$ of the variational formulation

$$
\int_{D}\left(\alpha_{r e g}^{2} \nabla Q \cdot \nabla v+Q v\right) d x=L^{\prime}(\Omega, \ell, \mu)(v n) \quad \forall v \in H^{1}(D),
$$

where $\alpha_{r e g}>0$ is a positive number (of the order of the mesh size $\Delta x$ ) which controls the regularization width around $\partial \Omega$. We check that $\theta=-Q n$ is indeed a descent direction since

$$
L^{\prime}(\Omega, \ell, \mu)(-Q n)=-\int_{D}\left(\alpha_{r e g}^{2}|\nabla Q|^{2}+Q^{2}\right) d x \leq 0 .
$$

It turns out that problem (29) will be useful for another reason when computing the shape derivative of the minimum thickness constraint (or of the minimum members' distance constraint). Indeed, its shape derivative, as furnished by Lemma 5.2, is non-local in the sense that the second integral of formula (22) involves the value of the normal component $\theta \cdot n$ at another point $p_{\partial \Omega}\left(x_{m}\right)$ which may be different from $x \in \partial \Omega$. Note in passing that it is not a contradiction with the Hadamard structure theorem (recalled in Section 2.2) since the shape derivative still depends only on the normal trace of $\theta$ on $\partial \Omega$, albeit in a non-local manner. In any case, it is not possible to easily extract a descent direction from (22) as we did in (28). The extension-regularization problem (29) will help us in this matter as we now explain on a specific example with a minimum thickness constraint.

Consider the optimization problem

$$
\begin{array}{ll}
\min _{\Omega \in \mathcal{U}_{a d}} & J(\Omega)=\int_{\Omega} f \cdot u d x+\int_{\Gamma_{N}} g \cdot u d s, \\
\text { s.t. } & P_{1}(\Omega)=\int_{\Omega} d x-\alpha_{V}|D|=0,\left(0<\alpha_{V}<1\right), \\
& P_{2}(\Omega)=P_{M i n T}(\Omega)=\int_{\partial \Omega} \int_{0}^{d_{\min }}\left[\left(d_{\Omega}\left(x_{m}\right)\right)^{+}\right]^{2} d \xi d x=0,
\end{array}
$$

for which we introduce the augmented Lagrangian (27), with shape derivative

$$
L^{\prime}(\Omega, \ell, \mu)(v n)=\int_{\Gamma} v(x)\left(-A e(u) \cdot e(u)-\ell_{1}+\mu_{1} P_{1}(\Omega)\right) d x-\ell_{2} P_{2}^{\prime}(\Omega)(v n)+\mu_{2} P_{2}(\Omega) P_{2}^{\prime}(\Omega)(v n)
$$

where

$$
\begin{aligned}
P_{2}^{\prime}(\Omega)(v n)= & \int_{\Gamma} \int_{0}^{d_{\min }} v(x)\left[H\left(\left(d_{\Omega}\left(x_{m}\right)\right)^{+}\right)^{2}+2\left(d_{\Omega}\left(x_{m}\right)\right)^{+} \nabla d_{\Omega}\left(x_{m}\right) \cdot \nabla d_{\Omega}(x)\right] d \xi d x \\
& -\int_{\Gamma} \int_{0}^{d_{\min }} v\left(p_{\partial \Omega}\left(x_{m}\right)\right) 2\left(d_{\Omega}\left(x_{m}\right)\right)^{+} d \xi d x
\end{aligned}
$$

In the above formula we restricted our attention to vector fields $\theta$ such that $\theta(x)=v(x) n(x)$, where $v(x)$ is a scalar field and $n(x)$ is the unit normal vector field, suitably extended to the whole computational domain $D$. Then, there is no difficulty in plugging formulas (31) and (32) as the right hand side of the variational formulation (29), the solution of which yields a valid descent direction. The non-locality of (32) is not an issue when it is used merely for computing the right hand side of (29). 


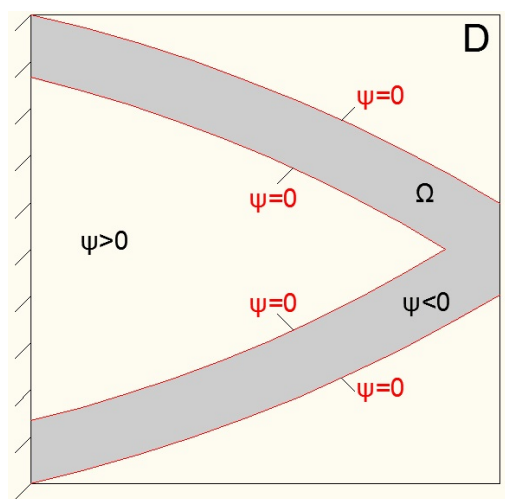

(a)

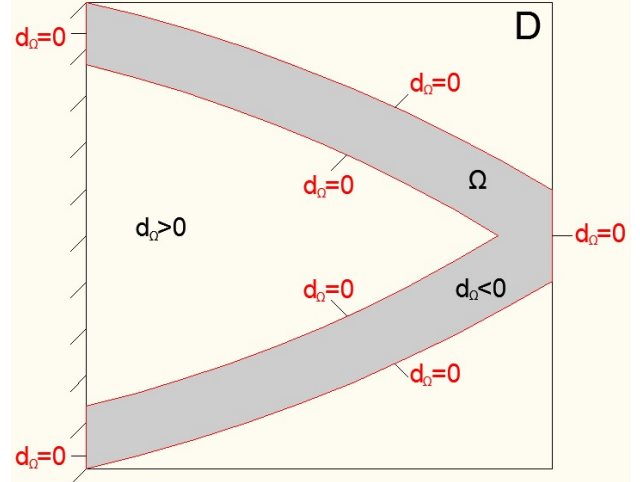

(b)

Figure 8: (a): level-set function $\psi$ used for the advection of the shape; (b): signed distance function $d_{\Omega}$.

\section{Numerical implementation}

In this section, we highlight some of the difficulties one may face when implementing the above methods and the solutions we have chosen for our numerical examples. The level set function, as well as the signed distance function, are discretized on a uniform rectangular mesh. Our remarks are thus pertaining to this framework.

\subsection{Construction of $d_{\Omega}$}

We emphasize again that the level-set function $\psi$, used for the description and advection of the shape, even if it is reinitialized to be a signed distance function, does not necessarily contain the correct information for the thickness of the structure. The signed distance function $d_{\Omega}$, that we use for the formulation of the constraints, refers to the true shape (including its part of the boundary lying on the border of the computational domain). In order to build $d_{\Omega}$, we take the level set function $\psi$, modify it by imposing $\psi=0$ at the points of the shape intersecting with the boundary of the working domain $D$ (see Figure 8) and reinitialize it to get the desired result. For the reinitialization, different algorithms can be used (fast marching, fast sweeping, Hamilton-Jacobi equation, etc... [33], [39], [52]). In Figure 9 we show the differences between the signed distance function $d_{\Omega}$ and the level-set function $\psi$.

Of course, if a symmetry condition for the shape $\Omega$ is enforced on part of the boundary of $D$, then we do not apply a Dirichlet boundary condition there for $d_{\Omega}$ but rather a symmetric boundary condition.

\subsection{Maximum thickness: integration along the rays}

To compute numerically the shape derivative $(20)$ of the maximum thickness functional $P_{\operatorname{MaxT}}(\Omega)$ we first have to identify the nodes of the mesh which "belong" to the boundary $\partial \Omega$ : any node of a cell cut by the zero level set of $\psi$ is considered as a boundary point. Second, we have to integrate along the rays starting from these points and finishing at the skeleton (cf. Definition 3.3). The direction of the ray is the normal vector at the boundary node. Starting from the boundary points, we advance by small steps (we have set the step to $0.5 \Delta x$, where $\Delta x$ is the unifrom mesh size) and use a quadrature formula for the numerical approximation of the term

$$
\int_{\operatorname{ray}(x) \cap \Omega} 2\left(d_{\Omega}(z)+d_{\max } / 2\right)^{-} \prod_{i=1}^{d-1}\left(1+d_{\Omega}(z) \kappa_{i}(x)\right) d z
$$

in (20). The principal curvatures $\kappa_{i}$ at the boundary points are computed using finite differences. Finally, to stop integrating along the ray we check the monotonicity of the signed distance function. When the monotonicity changes, we claim that we have reached the skeleton and the ray terminates at the corresponding point. 


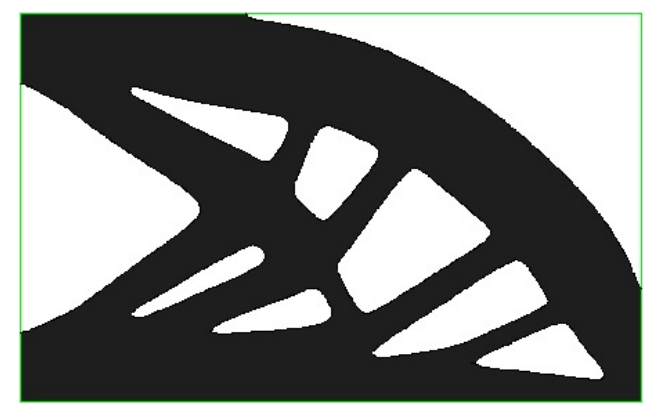

(a)

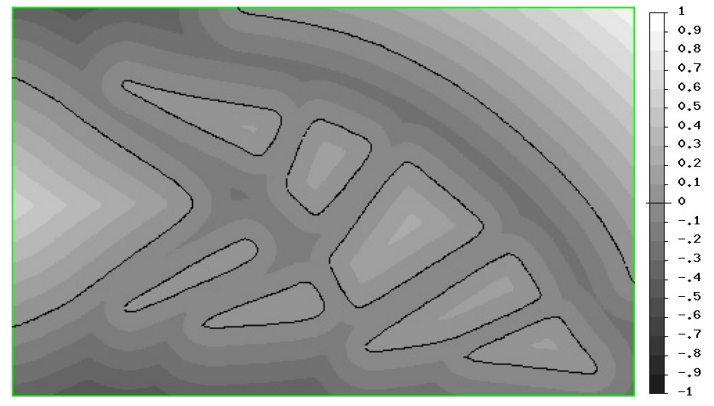

(b)

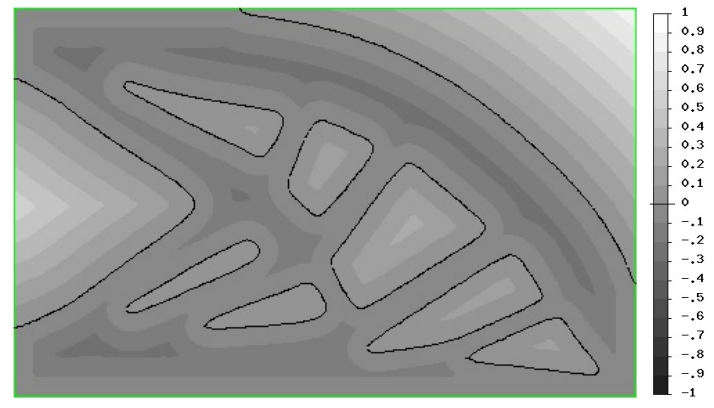

(c)

Figure 9: (a): shape in black $(\Omega)$; (b): iso-contours of the level-set function $\psi$ for the advection of the shape; (c): iso-contours of $d_{\Omega}$.

\subsection{Maximum thickness: "true", "Jacobian-free" and "approximate" shape derivatives}

In order to reduce the distortions close to junctions of structural members, we tried some simplifications on the shape derivative (20) of $P_{M a x T}$. In [4], we have shown that the Jacobian term $\prod_{i=1}^{d-1}\left(1+d_{\Omega}(z) \kappa_{i}(x)\right)$ in the shape derivative is always positive. Since the calculation of the curvature is not very accurate on a fixed mesh, we prefer to omit this term without changing the descent nature of the shape derivative, since the term

$$
\left(d_{\Omega}(\cdot)+d_{\max } / 2\right)^{-}
$$

in the integrand of (20) is also of constant sign. Moreover, neglecting this Jacobian term will have no influence for "flat" boundaries, where $\kappa_{i}(x) \equiv 0$, while the shape derivative will be overestimated if $\kappa_{i}(x) \geq 0$ and underestimated if $\kappa_{i}(x) \leq 0$, helping to avoid distortions at these regions. We call "true" formula the complete formula (20) and "Jacobian-free" formula the one where the Jacobian term is omitted, in agreement with [4]. Finally, we have considered a third formula, denoted "approximate", which gives very promising numerical results.

Let us now explain in detail the "approximate" formula. Starting from formula (21) for the shape derivative (which is a volume integral on the entire shape $\Omega$ ), we would like to substitute $d_{\Omega}^{\prime}(x)(\theta)$ with an explicit expression defined in the whole domain $\Omega$. However, such an expression exists only for the points on the boundary $\partial \Omega$ (see equation (10)). In a first step, we extend the expression of $d_{\Omega}^{\prime}(x)(\theta)$ on $\partial \Omega$ to the whole domain $\Omega$, i.e. we consider the approximation $d_{\Omega}^{\prime}(x)(\theta) \approx-\theta(x) \cdot n(x)=-w(x)$, for any $x \in \Omega$, where the exterior normal $n$ has been extended to the whole domain. Then, the approximation of the shape derivative of $P_{\operatorname{Max} T}(\Omega)$ reads

$$
P_{M a x T}^{\prime}(\Omega)(\theta) \approx-\int_{\Omega} 2 w(x)\left(d_{\Omega}(x)+d_{\max } / 2\right)^{-} d x .
$$

Note in passing that the approximate formula (33) does not satisfy the Hadamard structure theorem, i.e., is not in the classical form (5). The choice

$$
w(x)=+2\left(d_{\Omega}(x)+d_{\max } / 2\right)^{-}, \quad \forall x \in \Omega,
$$

which ensures that the right hand side of (33) is negative, gives a zero velocity for the points $x \in \partial \Omega$ and thus it is not useful. However, the approximate formula (33) of the shape derivative could be put as 


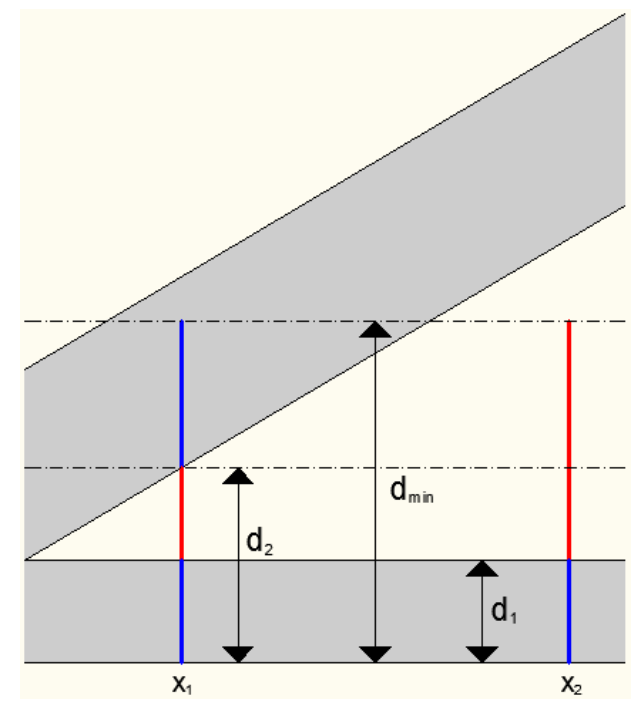

Figure 10: Offset points for $x_{1}, x_{2} \in \partial \Omega$ up to a distance $d_{\text {min }}$; violation areas of constraint (13) are depicted in red, while in blue areas the constraint is respected.

a right-hand side in the regularization equation (29), which would define another velocity with non-zero values on $\partial \Omega$. In such a case, the regularization parameter $\alpha_{\text {reg }}$ has to be chosen larger than its usual value of the order of $\Delta x$ (where $\Delta x$ is the uniform mesh size) and needs to be adjusted at each iteration so that the values of the new velocity field are not too small on $\partial \Omega$. In order to avoid disturbing significantly the shape gradients of the other terms in the augmented Lagrangian functional, we prefer to regularize the shape gradient of the maximum thickness constraint separately from the rest. For our numerical examples using (33), we have set

$$
\alpha_{r e g}^{2}=\left(2 \frac{\max _{\Omega}\left|d_{\Omega}(x)\right|}{\Delta x}\right)(\Delta x)^{2}
$$

for the regularization of the maximum thickness gradient.

An intuitive explanation of our heuristic choice is the following. The term $2\left(d_{\Omega}(x)+d_{\max } / 2\right)^{-}$ is non-zero only at regions violating the thickness constraint. Using the mathematically correct coarea transformation, this term influences significantly the highly curved parts. When the above approximation is used, the curvature information is not used. Instead what matters is the distance of a point $x \in \partial \Omega$ to the areas of thickness violation. Thus, using the "approximate" formula, the velocity due to the maximum thickness constraint for two points $x_{1}, x_{2} \in \partial \Omega$ lying close to each other, will not differ significantly. What is also important is that the direction of the velocity field will not change at any point, i.e. the final velocity field will always tend to reduce the thickness everywhere and thus it is a descent direction.

\subsection{Minimum thickness and minimum members' distance}

At this point let us emphasize that, by using the penalty functionals $P_{M i n T}(\Omega)$ and $P_{M M D}(\Omega)$, it is the values of the signed distance function that we penalize and not the "actual" thickness of a member, the latter being defined as the distance from a point $x \in \partial \Omega$ up to the next point on $\partial \Omega$ along the line starting from $x$ in the direction of $-n(x)$. Since the constraint is by essence non-local, this means that the derivative at a point will depend on the values of $d_{\Omega}(x)$ along the offset set. As a consequence, one can observe a significant difference in the velocity between points of equal "actual" thickness. In Figure 10, we show two bars in grey, the lower one being of constant thickness $d_{1}$. Starting from two points of its boundary $x_{1}$ and $x_{2}$ and advancing in the direction of $-n\left(x_{1}\right)$ and $-n\left(x_{2}\right)$ up to a distance $d_{\text {min }}$, we show in blue colour the parts where the minimum thickness constraint (13) is satisfied and in red the parts where it is violated. Not only is the length of the red parts different, but also the values of the signed distance function differ along them, so that even in case that $d_{\min }=d_{2}$, point $x_{2}$ would have a higher contribution in $P_{M i n T}$ than point $x_{1}$ and also a higher shape gradient magnitude.

Another strange effect is the implied motion of points that do not violate the constraint! For instance, Figure 11 displays an offset set crossing the skeleton formed between two bars. In this case, one part of 


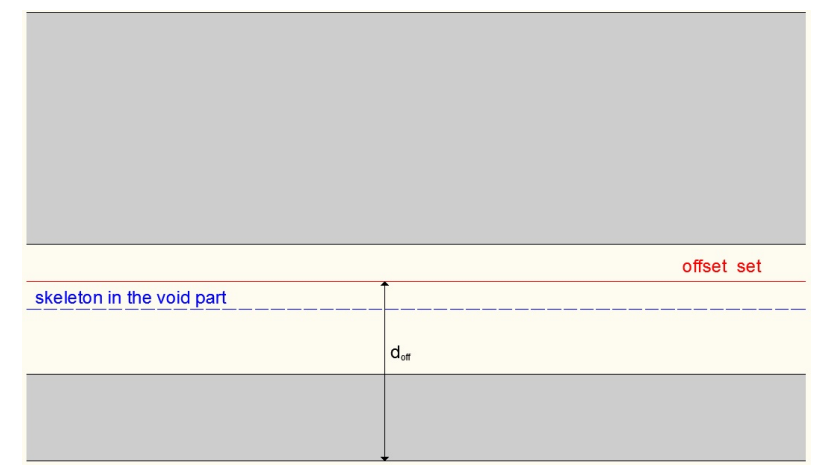

Figure 11: Crossing of the skeleton formed in the void part.

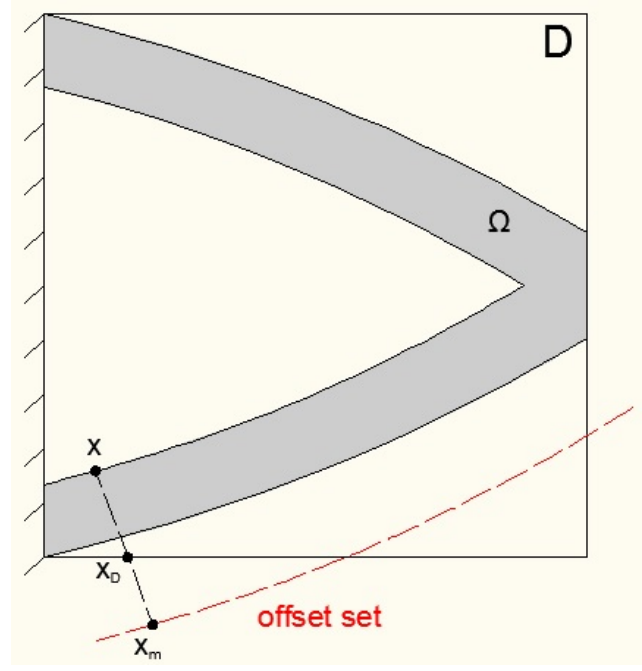

Figure 12: Offset set crossing the boundary of the working domain $\partial D$.

the shape derivative is set on the projection of the offset set on the boundary and thus the second bar is also affected due to the thickness violation of the first bar. One possible choice to reduce the importance of the above observations is to apply a continuation method and increase progressively the offset distance up to the value $d_{\min }$ (we have not implemented this strategy in this work).

Another difficulty appears when some part of the offset set lies outside the working domain $D$, i.e. when we need to extend $d_{\Omega}(x)$. Suppose that we start from a point $x \in \partial \Omega$ and start moving in the direction $-n(x)$ up to the offset set (see Figure 12). Once we cross the boundary of the working domain $\partial D$, we need to account for the values of $d_{\Omega}$ outside $D$. In case we have symmetry conditions, we just have to change the sign of some component of the normal vector and to continue moving in the new direction, i.e. the boundary acts like a mirror. Else, if the whole shape is included in the working domain $D$, one possibility is to consider that it is surrounded by void. Then, to compute exact values of $d_{\Omega}(x)$ would require to have a zone of non-optimizable weak material of thickness at least $d_{\text {min }}$ around the domain $D$. A simplification, in order to avoid technical difficulties, is to use instead the Euclidean distance between the offset point $x_{m}$ and the point where the line crosses $\partial D$, denoted $x_{D}$. Hereafter, we approximate the value of $d_{\Omega}\left(x_{m}\right)$ by

$$
d_{\Omega}\left(x_{m}\right)=d_{\Omega}\left(x_{D}\right)+d\left(x_{m}, x_{D}\right)
$$

where $d\left(x_{m}, x_{D}\right)=\left|x_{m}-x_{D}\right|$ stands for the usual Euclidean distance.

\section{Numerical examples}

All examples in this section have been coded in ESI Group's SYSTUS ${ }^{\circledR}$ finite element code [49]. A cartesian mesh has been used both for solving the elasticity system and as a support for the level-set function describing the shape. For the elasticity analysis, we have used Q1 finite elements. The Young 


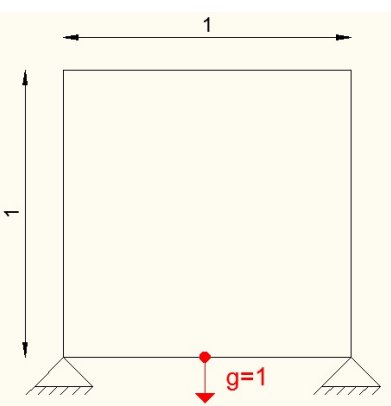

(a)

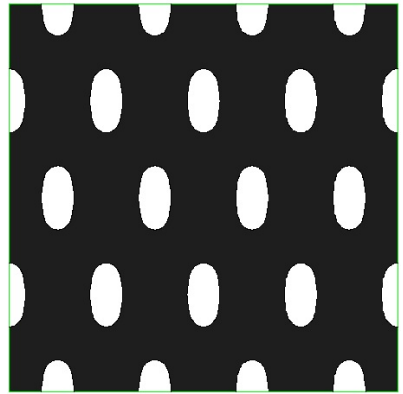

(b)

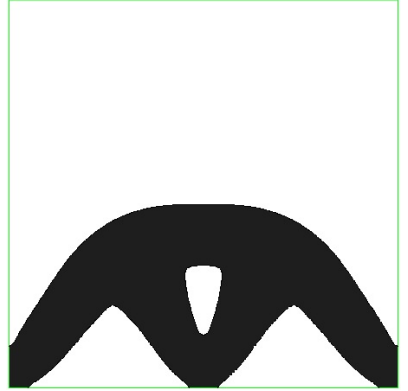

(c)

Figure 13: 2d arch; (a): boundary conditions; (b): initialization; (c): optimized shape for the optimization problem (34).

modulus of the elastic material $E$ is normalized to 1 and the Poisson ratio $\nu$ is set to 0.3 . The "ersatz material" is considered to have the same Poisson ratio, while its Young modulus is set to $10^{-3}$. In all problems containing a maximum thickness constraint, the regularized penalty functional (18) has been used for $P_{\text {MaxT }}$. When imposing one and only one thickness constraint, the corresponding feature size is represented by a bar (with the correct size) below the figure: its color is black if it is a constraint for the solid part, while it is grey for the void part. When both minimum and maximum thickness constraints are imposed, there are two black bars.

\subsection{Maximum thickness}

\subsubsection{2d arch}

The first example is a two-dimensional arch-like structure, clamped at its lower left and right corners and with a unitary force applied at the middle of its lower part (see Figure 13). Due to symmetry, only half of the domain is used and it is discretized by $80 \times 160$ elements. As a first step we solve the optimization problem

$$
\begin{aligned}
\min _{\Omega \in \mathcal{U}_{a d}} & \int_{\Omega} d x \\
\text { s.t. } & \int_{\partial \Omega} g \cdot u d s \leq g_{\text {max }}^{1},
\end{aligned}
$$

where $u$ is the solution of $(7)$ and $g_{\max }^{1}=5$. The initialization and the optimized shape are shown in Figure 13.

We suppose now that this optimized shape violates a constraint of maximum thickness. A first idea to treat this problem is to impose the constraint in a second step, i.e. after that the shape has been optimized without imposing any thickness restriction. Then, the optimized structure of Figure 13 serves as an initial guess for the optimization problem

$$
\begin{aligned}
\min _{\Omega \in \mathcal{U}_{a d}} & \int_{\Omega} d x \\
\text { s.t. } & \int_{\partial \Omega} g \cdot u d s \leq g_{\text {max }}^{1}, \\
& P_{\text {Max }}(\Omega) \leq g_{\text {max }}^{2},
\end{aligned}
$$

where $g_{\max }^{1}=5, g_{\max }^{2}=d_{\max } / 2$ and $P_{M a x T}(\Omega)$ is given by (18). The optimized shape and the final thickness violation $\left(\left|d_{\Omega}(x)-d_{\max } / 2\right|\right)^{-}$, using the "true" formula, are depicted in Figure 14 . We shall be satisfied with a thickness violation of the order of the mesh size, since this is the order of accuracy for the computation of the signed distance function. One can observe that the optimized shape contains some significantly curved regions, which serve to tackle the thickness constraint, while the size of regions that are far from violating the constraint increased in order to satisfy the compliance constraint. The volume of the optimized shape increased from 0.1354 to 0.1384 .

The idea of imposing the maximum thickness constraint in a second step is quite natural, however it presents several drawbacks. First of all, especially in 2 d, the initially optimized shape usually has a much simpler topology compared to its initialization. Therefore, the algorithm will satisfy the constraint under much less freedom, as far as topology optimization is concerned, compared to the case when the constraint 


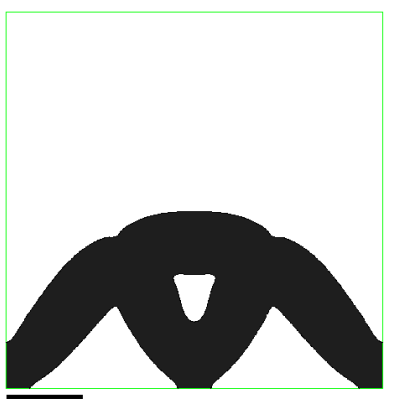

(a)

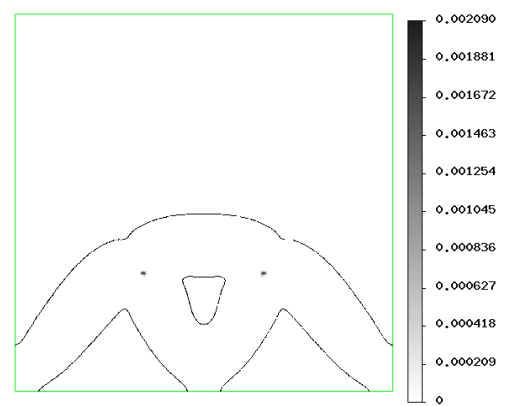

(b)

Figure 14: 2d arch using the "true" formula; (a): optimized shape; (b): violation of the constraint $\left|\left(d_{\Omega}(x)-d_{\max } / 2\right)^{-}\right|$, for the problem $(35)$ and $d_{\max }=0.2$.

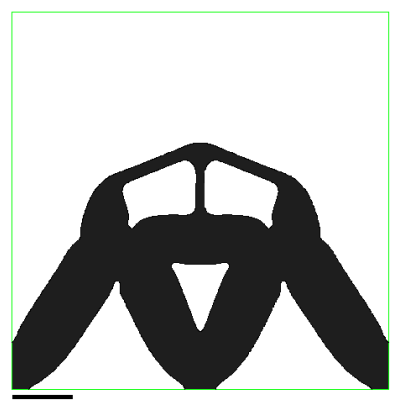

(a)

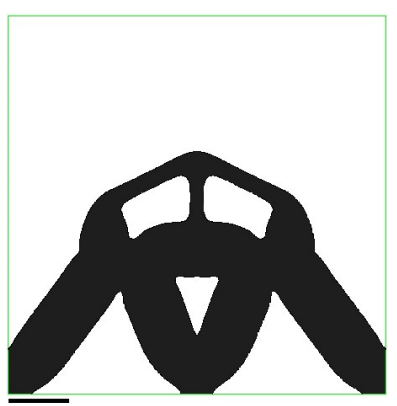

(b)

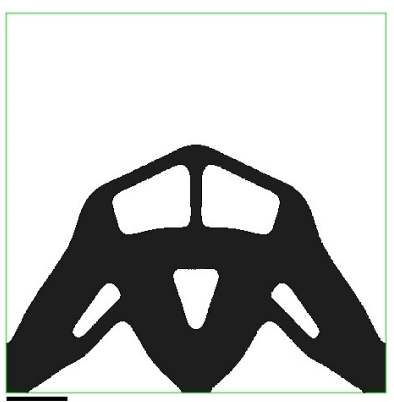

(c)

Figure 15: 2d arch; Optimized shapes for problem (35) and $d_{\max }=0.16$ using the (a): "true"; (b): "Jacobian-free" and (c): "approximate" formulas.

is applied since the beginning of the optimization process and it will probably result in curvy parts close to junctions of structural members. Besides, it is highly possible that starting from a shape with reduced topology, a solution to the problem (35) does not exist, or at least the optimization algorithm does not find a feasible solution. This is the case, for example, when problem (35) is solved for $d_{\max }=0.16$ and the previous strategy is followed.

Therefore, it seems natural to apply the maximum thickness constraint since the beginning and hope that starting with a complicated enough topology, the algorithm will arrive at a feasible shape. Starting with the initialization of Figure 13, we show in Figure 15 the results for such a case, using all three formulas for the shape derivative of $P_{\operatorname{Max} T}(\Omega)$. We can see in fact that the final topology is more complicated. The existence of many holes at the time the thickness constraint is applied, endows the algorithm with more flexibility in finding a feasible shape.

Although the optimized shapes of Figure 15 are almost equivalent with respect to the value of the objective fuction (see Table 1), we observe that less distortions close to joints appear when the "Jacobianfree" formula is used instead of the "true" one. Furthermore, they nearly disappear using the "approximate" formula. We have verified this tendency with several numerical results (see [29], pp. 73-101). For this reason, we have used the "approximate" formula for all numerical examples in the sequel.

In Figure 16 we show the results of problem (35) for $g_{\max }^{1}=5$ and for different values of $g_{\max }^{2}=$ $d_{\max } / 2$. Although we expect in general that the volume of the optimal shape increases when the maximum

Table 1: Results for the optimized shapes of Figure 15.

\begin{tabular}{|c|c|c|c|}
\hline & Volume & Compliance & $P_{\operatorname{Max} T}(\Omega)$ \\
\hline Without thickness restriction & 0.135 & 5.0 & - \\
\hline "true" formula $\left(d_{\max }=0.16\right)$ & 0.157 & 5.0 & 0.079 \\
\hline "Jacobian-free" formula $\left(d_{\max }=0.16\right)$ & 0.155 & 5.0 & 0.080 \\
\hline "approximate" formula $\left(d_{\max }=0.16\right)$ & 0.155 & 5.0 & 0.080 \\
\hline
\end{tabular}




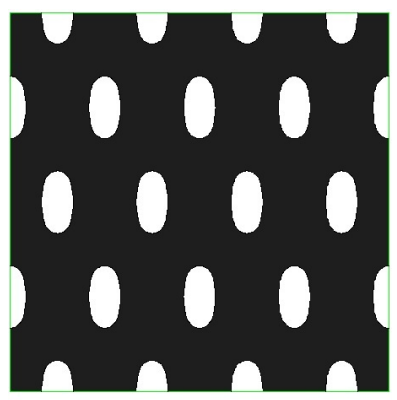

(a)

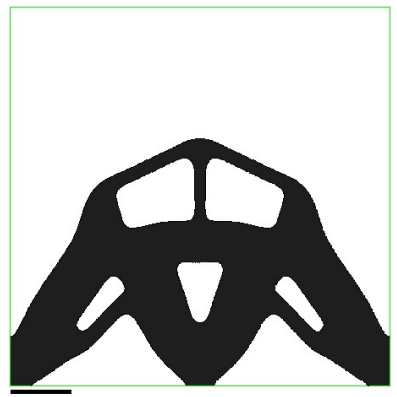

(d)

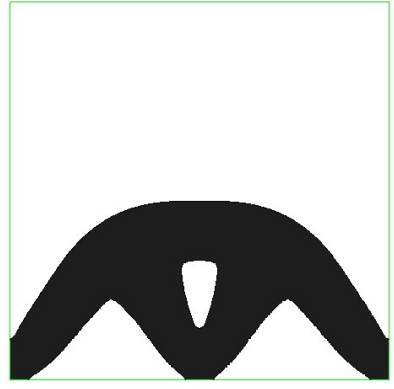

(b)

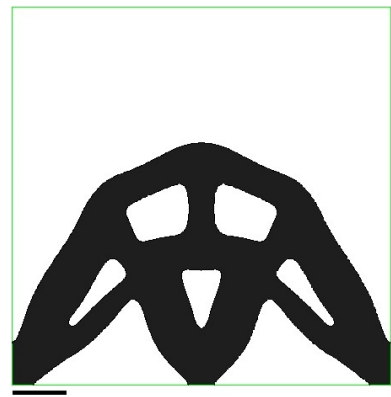

(e)

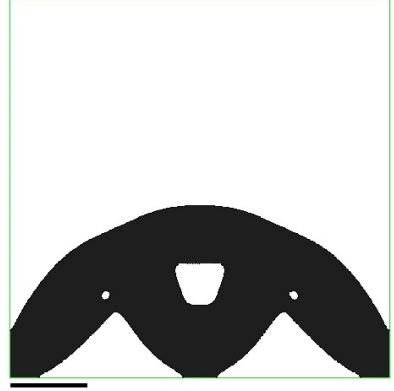

(c)

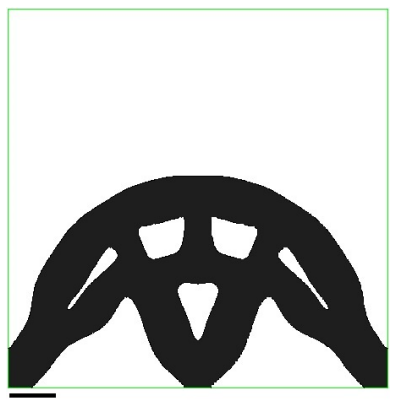

(f)

Figure 16: 2d arch; (a): initialization; optimized shapes for the optimization problem (35), using the "approximate" formula (b): without thickness restriction; (c): for $d_{\max }=0.20$; (d): for $d_{\max }=0.16$; (e): for $d_{\max }=0.14 ;(\mathrm{f})$ : for $d_{\max }=0.12$.

Table 2: Optimized 2d MBB beam.

\begin{tabular}{|c|c|c|c|}
\hline & Volume & Compliance & $P_{\operatorname{Max} T}(\Omega)$ \\
\hline Without thickness restriction & 1.881 & 40.0 & - \\
\hline$d_{\max }=0.30$ & 1.845 & 40.0 & 0.149 \\
\hline$d_{\max }=0.25$ & 1.900 & 39.9 & 0.125 \\
\hline$d_{\max }=0.20$ & 2.155 & 39.7 & 0.102 \\
\hline
\end{tabular}

thickness limit $d_{\max }$ decreases, this is not always true since plenty of local minima may exist. We also observe that when $d_{\max }$ is set to 0.12 , the optimized shape contains curved members. As we have mentioned before, this situation could possibly be avoided by starting with a more complicated topology. In Figure 17 we see the optimized shapes for $d_{\max }=0.12$, starting from initializations with more holes.

\subsubsection{2d MBB beam}

The next two-dimensional example for the maximum thickness constraint is the benchmark MBB beam. The dimensions of the enclosing box $D$ are $6 \times 1$ and a unitary vertical load is applied at the middle of its top edge (see Figure 18). Due to symmetry, half of the domain is considered and is discretized by $240 \times 80$ elements. The initial and the optimized shapes for problem (34) and $g_{\max }^{1}=40$ are shown on Figure 19. Using the same initialization, the optimized shapes and the violation of the thickness constraint for problem (35) and for different values of $d_{\max }$ are plotted in Figure 20. The convergence diagrams are shown in Figure 21 and the optimal numerical values in Table 2.

\subsubsection{3d box}

The last example for the maximum thickness constraint is a three dimensional $2 \times 2 \times 1$ box-like structure, clamped at its four lower corners and with a unit vertical load applied at the middle of its bottom face (see Figure 22). Here again, by symmetry, one quarter of the structure is used for the analysis and is discretized by $30 \times 30 \times 30$ elements. The initialization and the optimized shape for problem (34) and $g_{\text {max }}^{1}=32$ are shown in Figure 23. The optimized shapes for $g_{\max }^{1}=32$ and $d_{\max }=0.60$ and $d_{\max }=0.40$ 


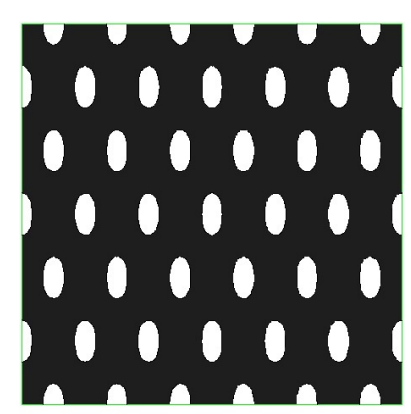

(a)

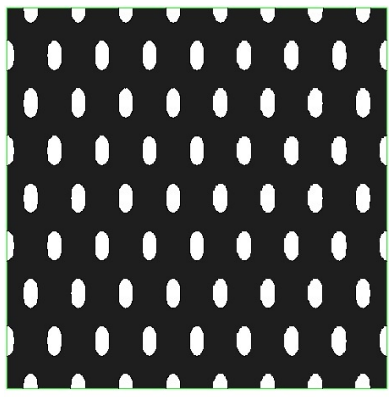

(d)

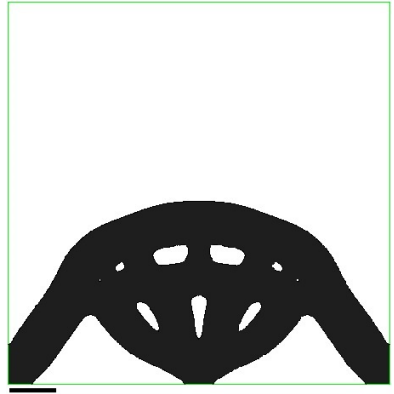

(b)

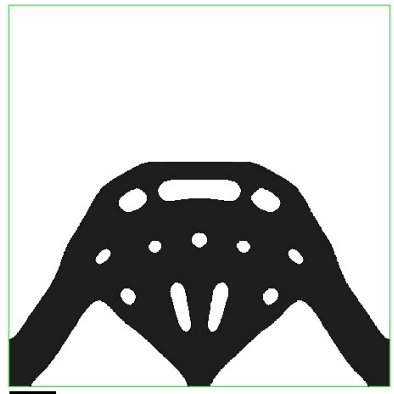

(e)

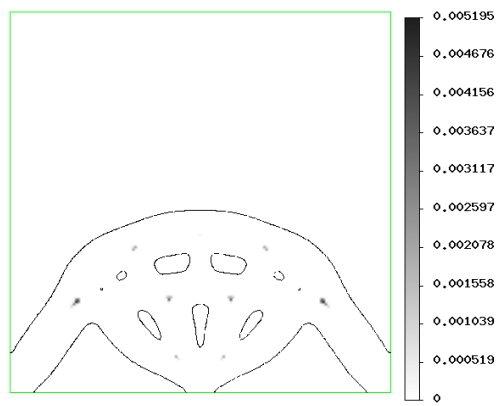

(c)

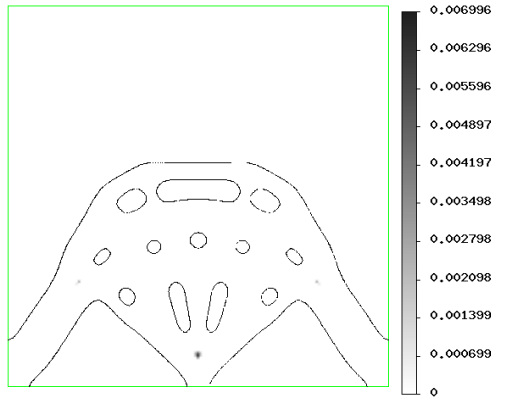

(f)

Figure 17: 2d arch using the "approximate" formula; (a),(d): initialization; (b),(e): optimized shape for the optimization problem (35) and $d_{\max }=0.12 ;(\mathrm{c}),(\mathrm{f})$ : violation of the constraint $\left|\left(d_{\Omega}(x)-d_{\max } / 2\right)^{-}\right|$.

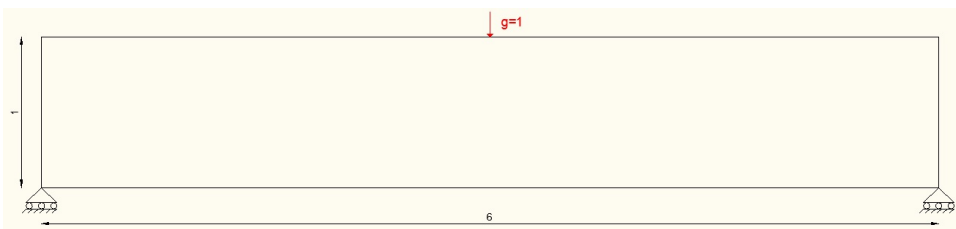

(a)

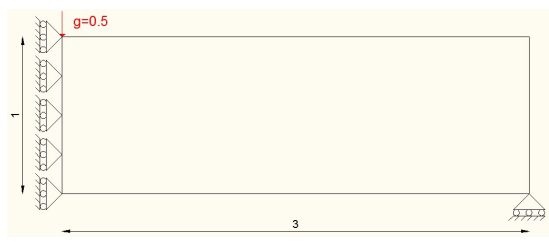

(b)

Figure 18: Boundary conditions for a 2d MBB beam; (a): full-domain; (b): half-domain.

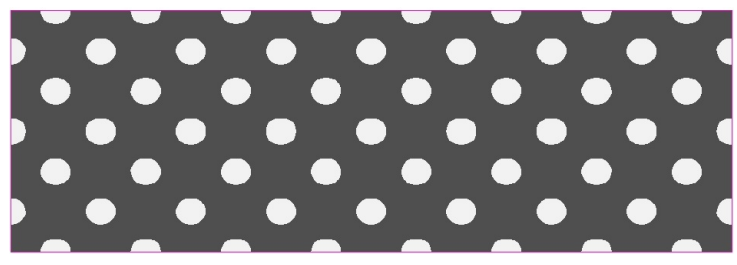

(a)

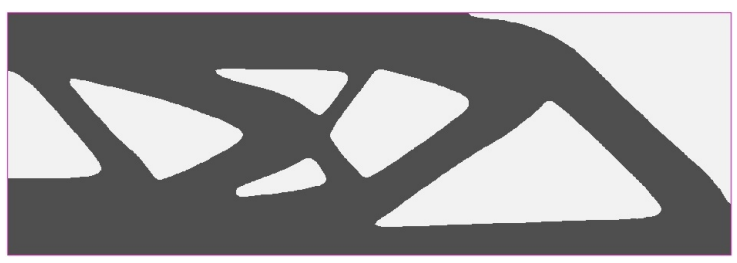

(b)

Figure 19: 2d MBB beam; (a): initialization; (b): optimized shape, for problem (34) (no thickness constraint) and $g_{\max }^{1}=40$. 

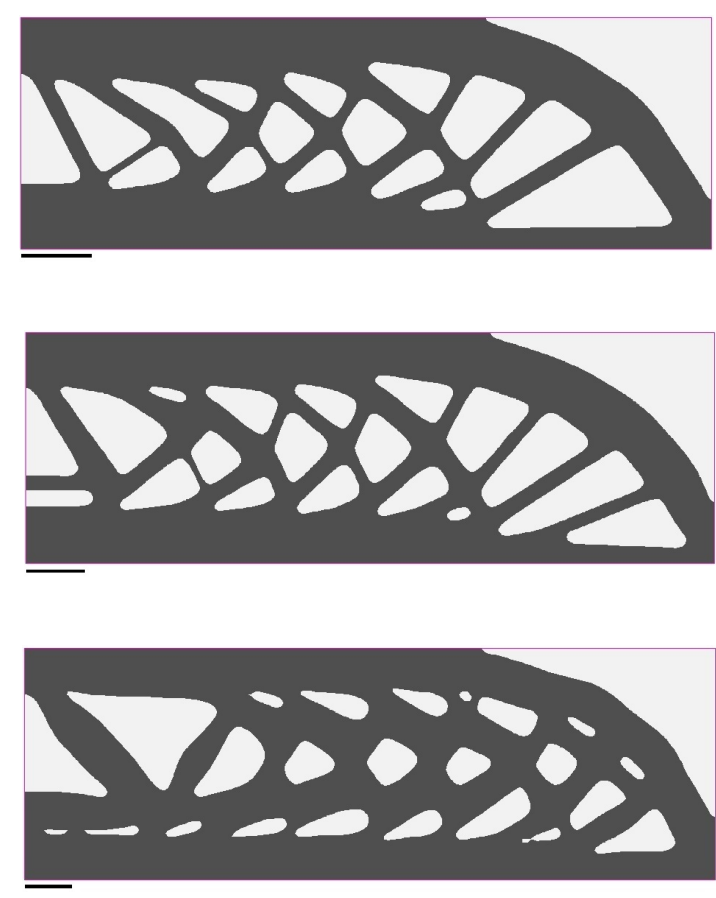

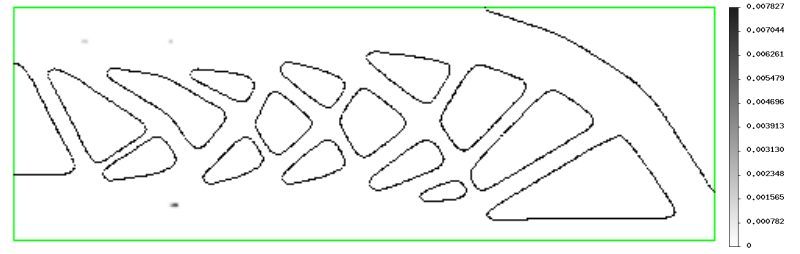

(a)

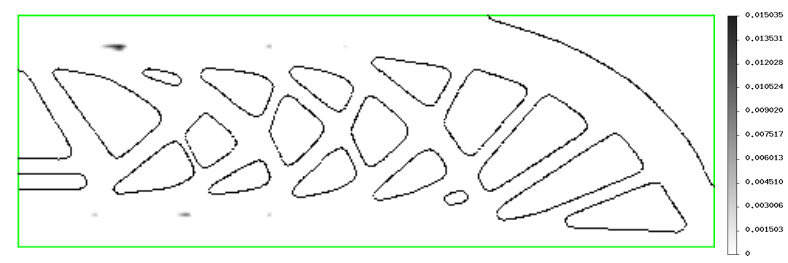

(b)

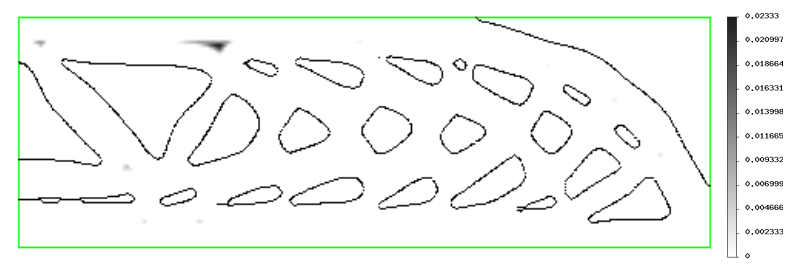

(c)

Figure 20: 2d MBB beam, using the "approximate" formula; optimized shapes for problem (35) and maximum thickness violation $\left(\left|\left(d_{\Omega}(x)-d_{\max } / 2\right)^{-}\right|\right)$for $(\mathrm{a}): d_{\max }=0.30 ;(\mathrm{b}): d_{\max }=0.25 ;(\mathrm{c}): d_{\max }=$ 0.20 .

Table 3: Optimized 3d box.

\begin{tabular}{|c|c|c|c|}
\hline & Volume & Compliance & $P_{\text {MaxT }}(\Omega)$ \\
\hline Without thickness restriction & 0.303 & 32.0 & - \\
\hline$d_{\max }=0.60$ & 0.281 & 32.0 & 0.234 \\
\hline$d_{\max }=0.40$ & 0.326 & 32.0 & 0.200 \\
\hline
\end{tabular}

are plotted in figures 24 and 25 correspondingly. The optimal numerical values are shown in Table 3 . We see that the optimized shape for $d_{\max }=0.60$ is lighter than the one obtained without a thickness constraint which proves that the shape of Figure 23 corresponds to a local and not global minimum.

\subsection{Minimum Thickness}

\subsubsection{2d cantilever}

We now turn to minimum thickness constraints and, as before, we shall test different strategies according to the type of chosen initialization. Our model test case is a $3.2 \times 2$ two-dimensional cantilever, discretized by $160 \times 100$ elements, clamped at its left part and with a unitary force applied at its lower-right corner (see Figure 26(a)). Starting with the initialization of Figure 26(b) and solving problem (34) (without thickness constraints) for $g_{\max }^{1}=60$, we get after 200 iterations the optimized shape of Figure 27(a).

We suppose now that we want to avoid thin features that appear in this shape. We define a minimum thickness size $d_{\min }$ and solve the optimization problem

$$
\begin{aligned}
\min _{\Omega \in \mathcal{U}_{a d}} & \int_{\Omega} d x \\
\text { s.t. } & \int_{\partial \Omega} g \cdot u d s \leq g_{\text {max }}^{1},
\end{aligned}
$$

Once more, we need to choose whether the thickness constraint will be applied since the beginning, or in a second step, after an optimized shape for problem (34) has been obtained. Contrary to the maximum 


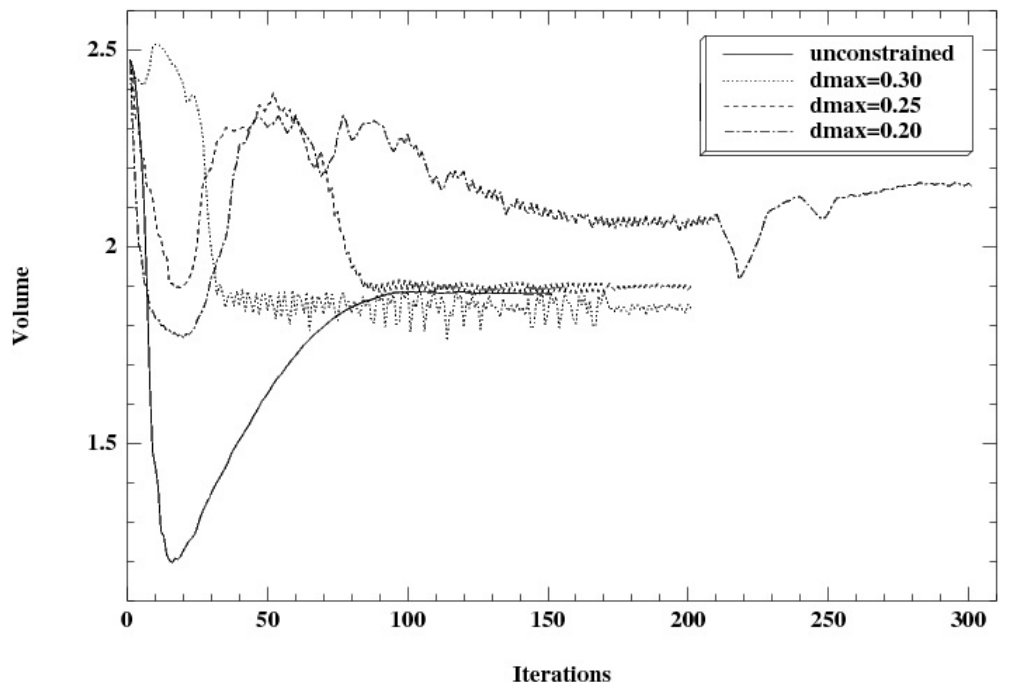

(a)

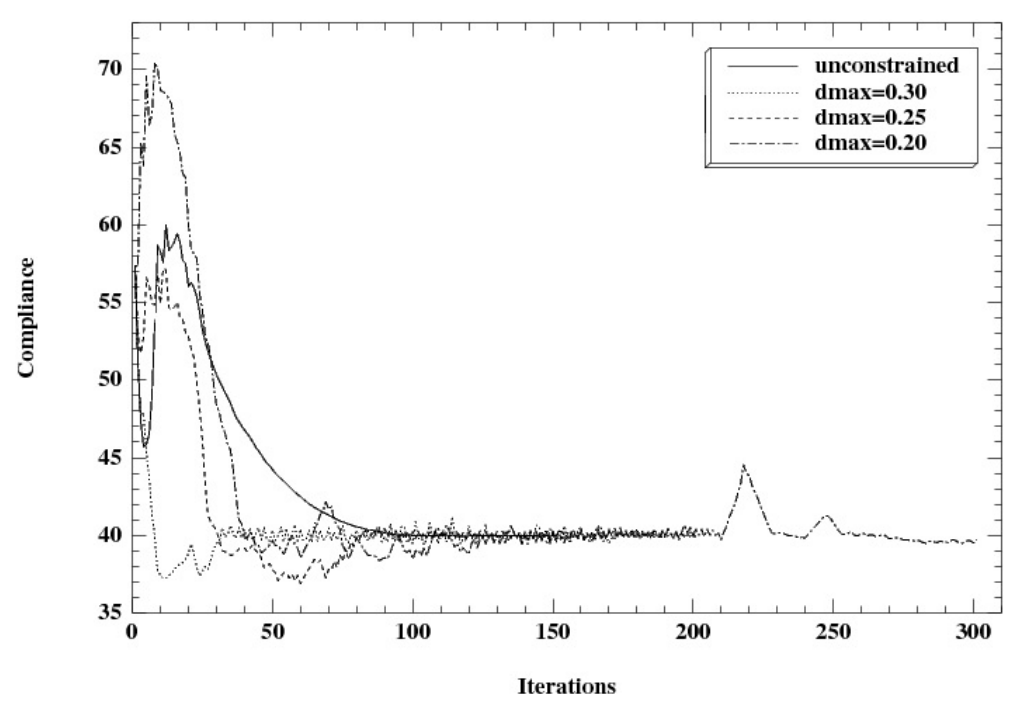

(b)

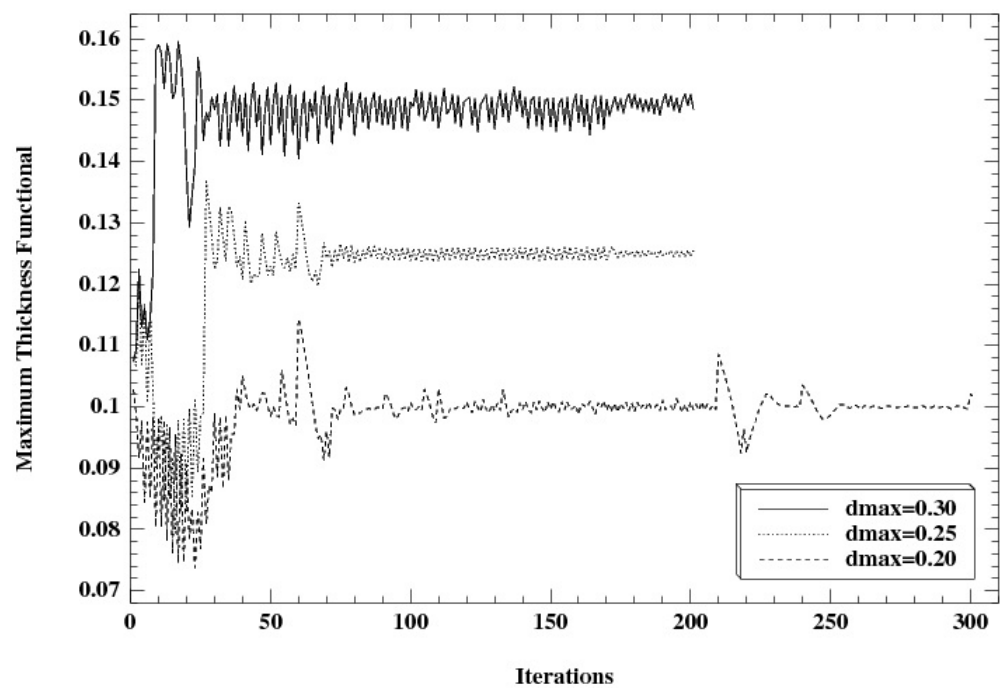

(c)

Figure 21: Convergence diagrams for the (a): volume; (b): compliance; (c): maximum thickness functional, for the results of figures 19 and 20. 


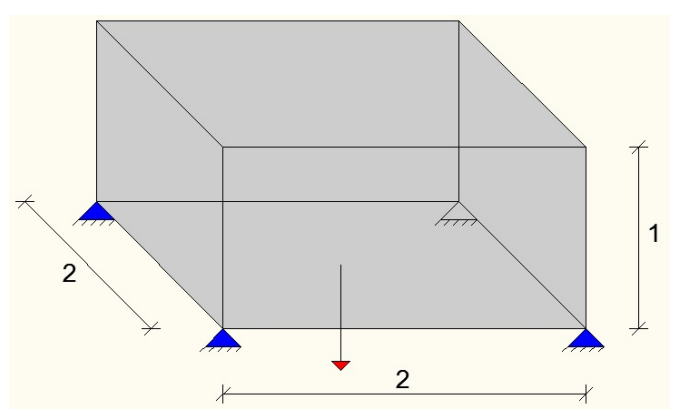

Figure 22: Boundary conditions for a 3d box.

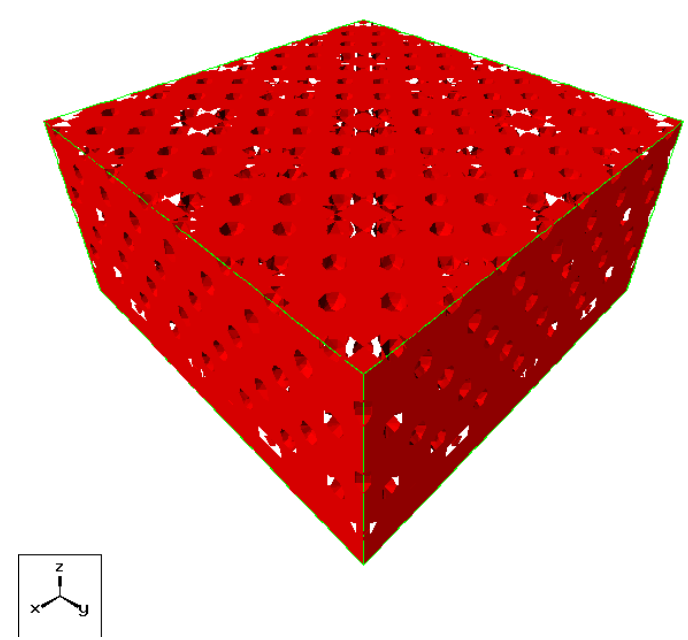

(a)
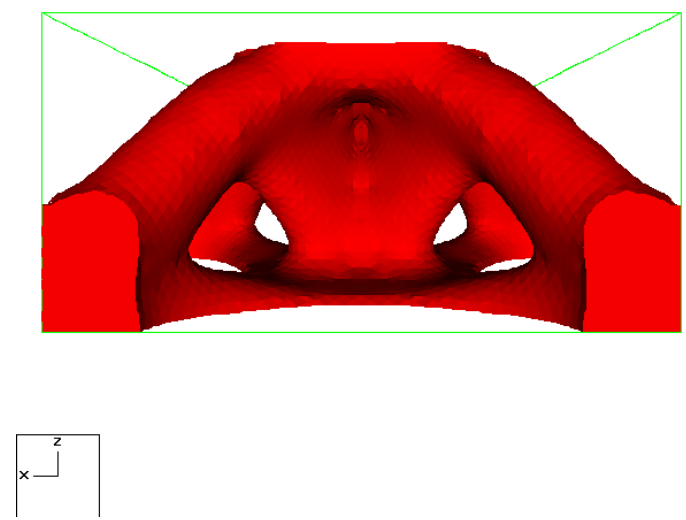

(c)

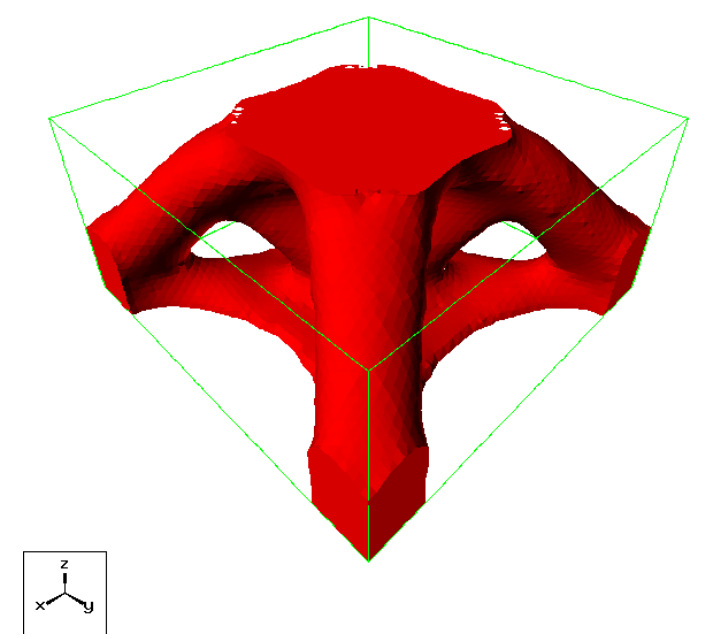

(b)

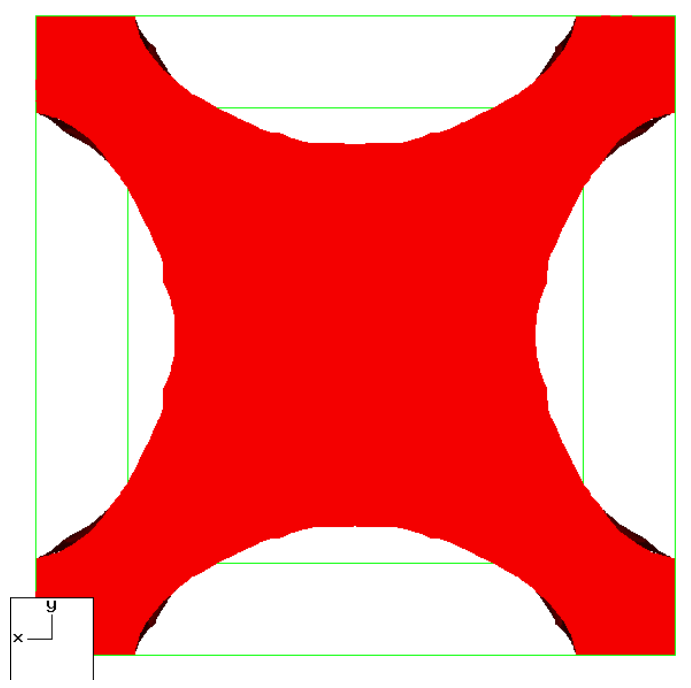

(d)

Figure 23: 3d box; (a): initialization; (b)-(d): different views of the optimized shape for problem (34) (without thickness constraints) and $g_{\max }^{1}=32$. 


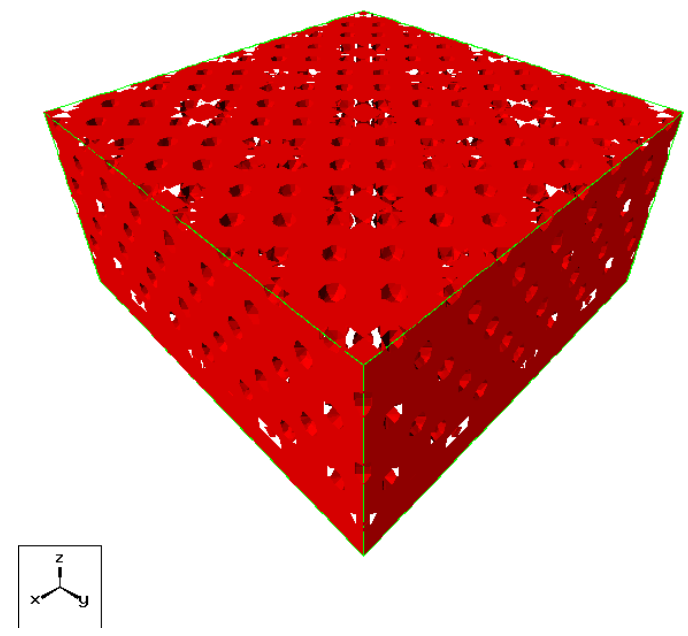

(a)
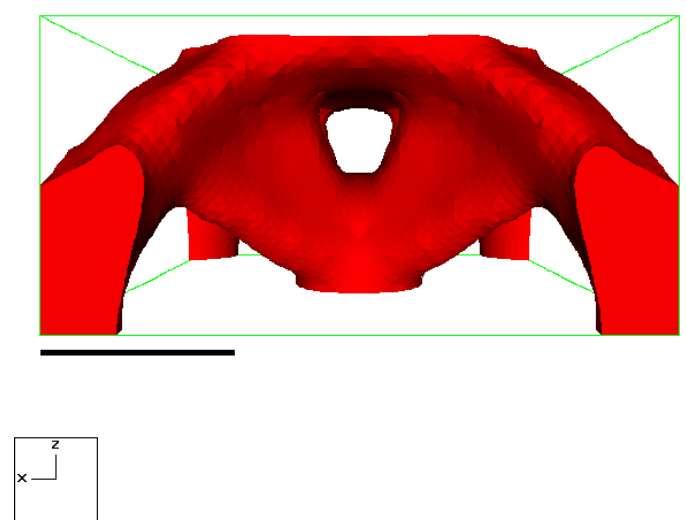

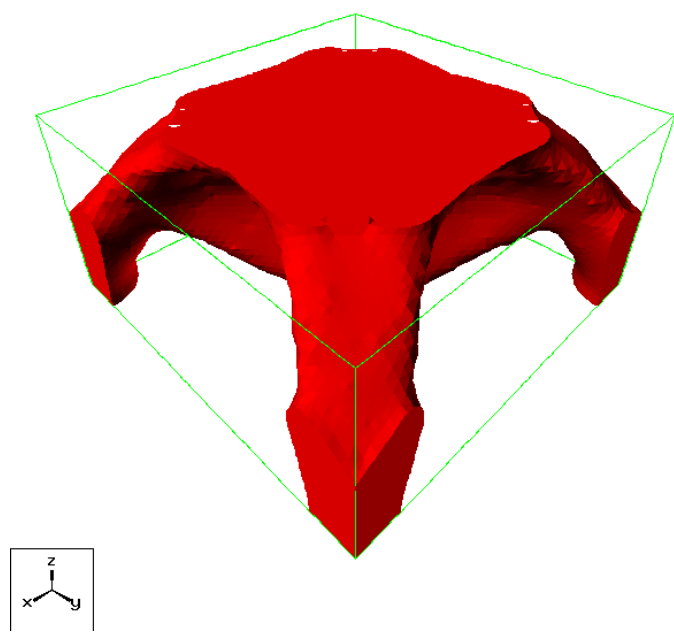

(b)

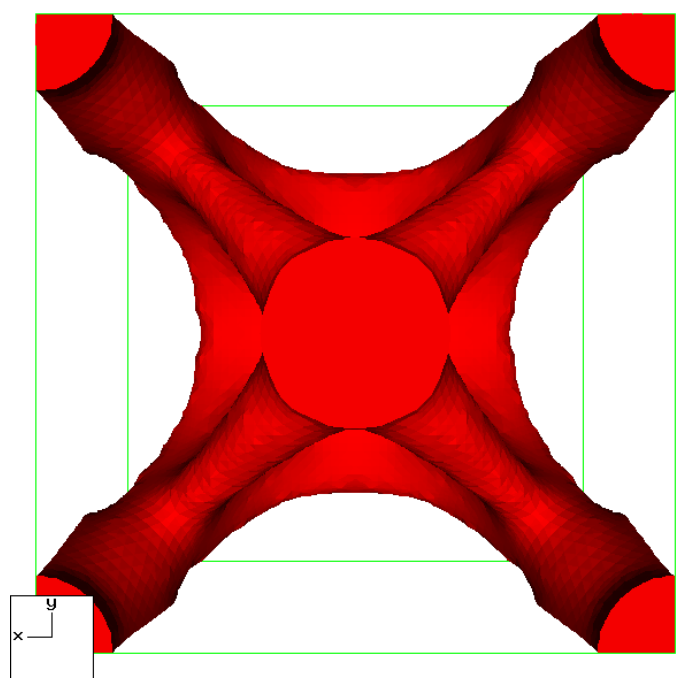

(d)

Figure 24: 3d box, using the "approximate" formula; (a): initialization; (b)-(d): different views of the optimized shape for problem (35), $g_{\max }^{1}=32$ and maximum thickness limit $d_{\max }=0.60$. 


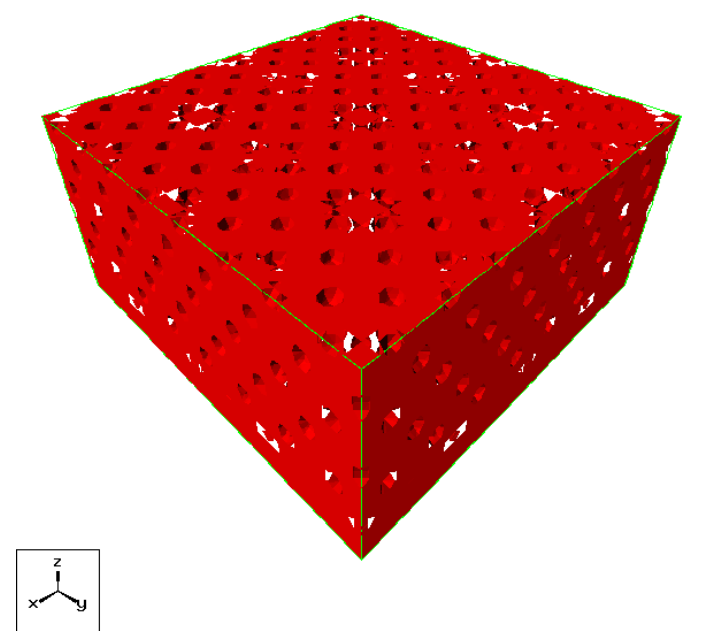

(a)
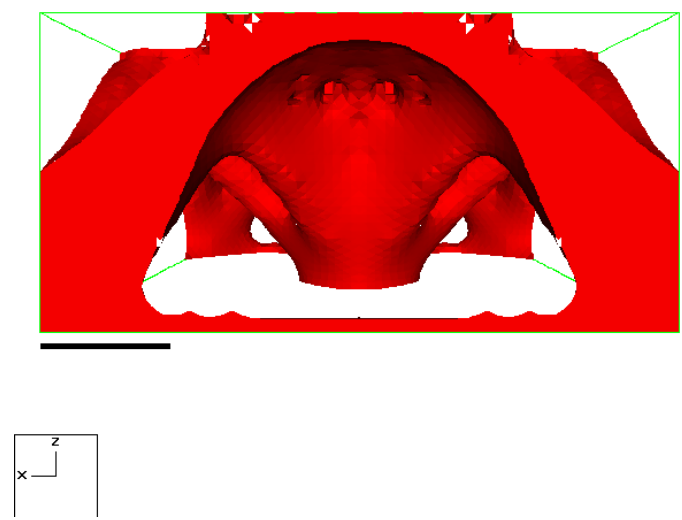

(c)

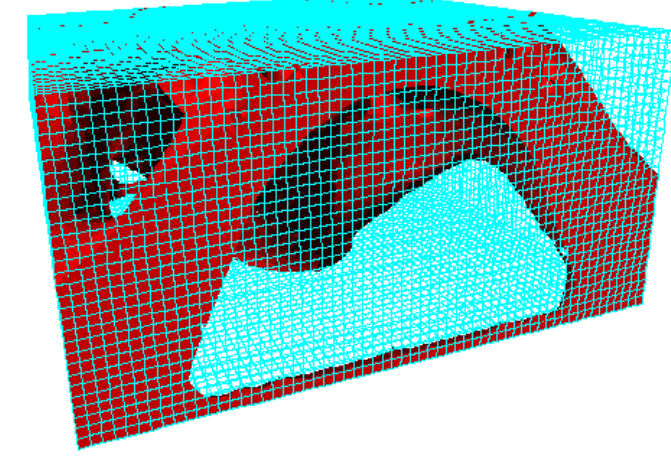

$x_{x-y}^{2}$

(e)

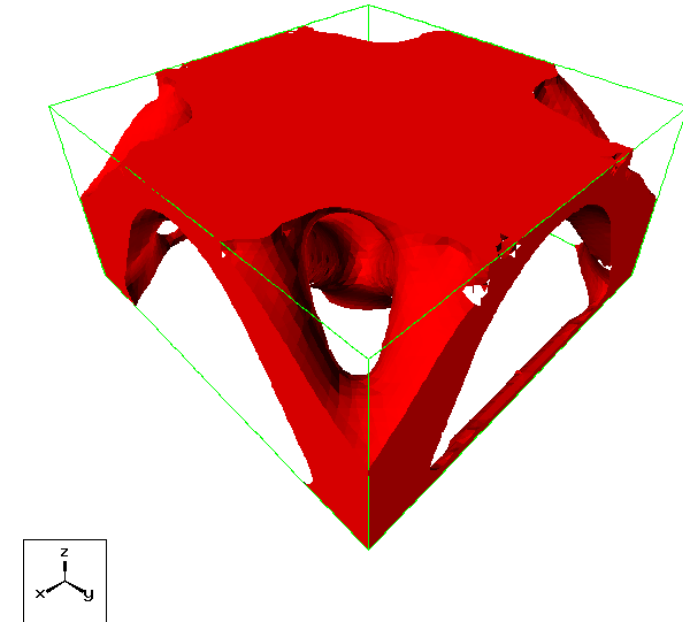

(b)

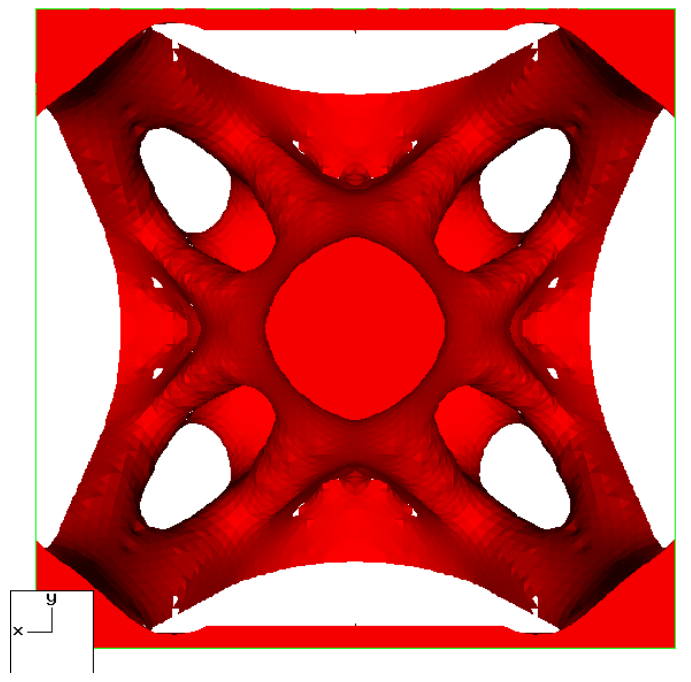

(d)

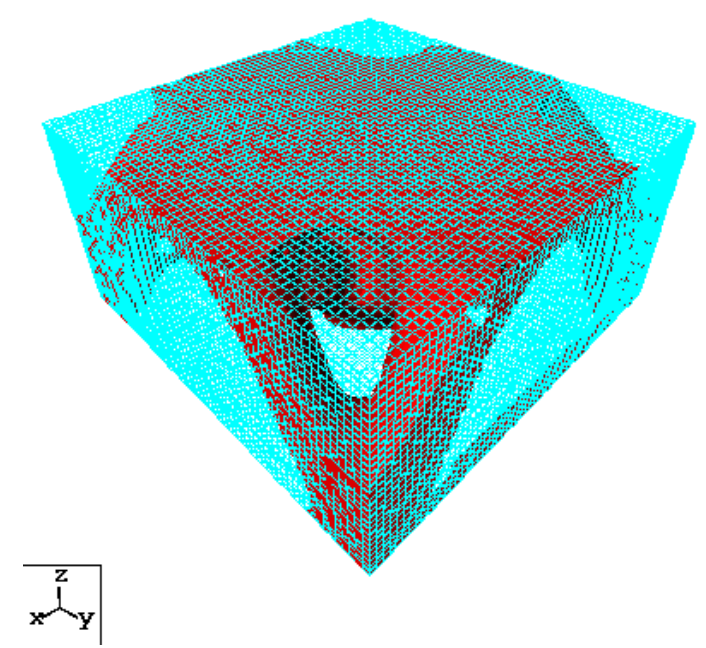

(f)

Figure 25: 3d box, using the "approximate" formula; (a): initialization; (b)-(d): different views of the optimized shape; (e)-(f): finite element discretized topologies, for problem (35), $g_{\max }^{1}=32$ and maximum thickness limit $d_{\max }=0.40$. 


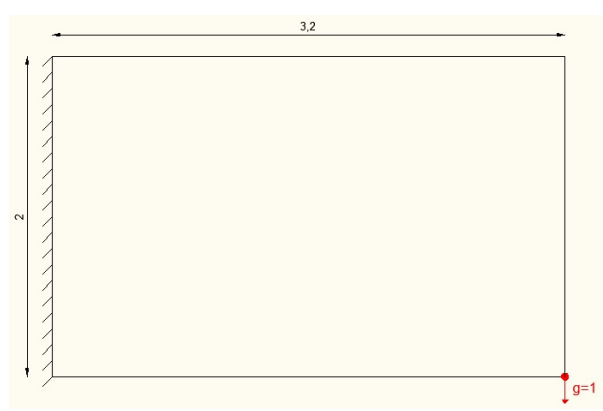

(a)

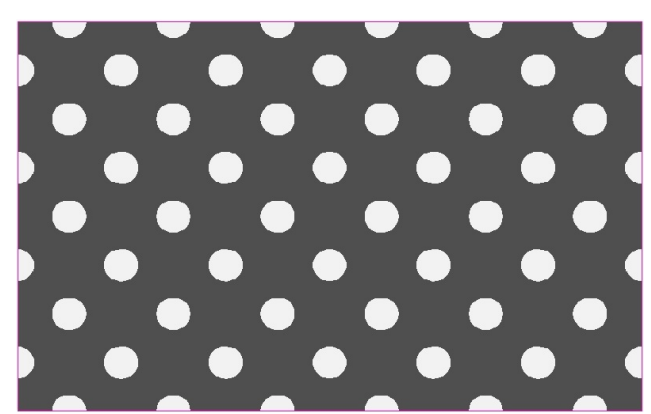

(b)

Figure 26: 2d cantilever; (a): boundary conditions; (b): initialization.

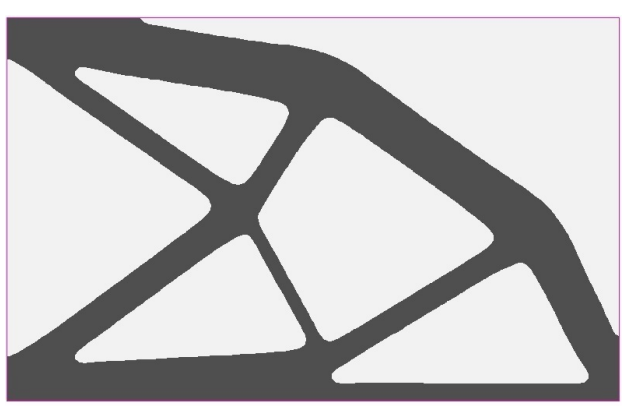

(a)

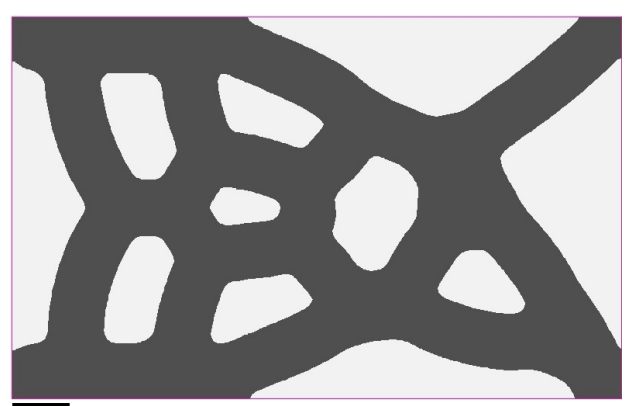

(b)

Figure 27: 2d cantilever; optimized shapes (a): for problem (34) (without thickness constraints) and $g_{\max }^{1}=60 ;(\mathrm{b})$ : for problem $(36), g_{\max }^{1}=60$ and minimum thickness limit $d_{\min }=30$ imposed since the beginning of the optimization algorithm (cf. Remark 8.1).

thickness case, it seems more natural to choose the second strategy for this constraint. The reason is that the penalty functional $P_{\text {MinT }}(\Omega)$ in (36) can hinder the merging of holes or the disappearance of thin bars. This tendency depends highly on the parameters of the optimization algorithm and their update strategy. Thus, for the first strategy, we shall in general expect the existence of parts in the optimized shape with negligible mechanical importance. This is the case in the optimized shape of Figure 27(b), where the minimum thickness constraint has been applied since the beginning of the optimization algorithm. Evidently, the upper-right bar of the shape has negligible mechanical contribution, but it cannot be removed because of the minimum thickness constraint (see Remark 8.1 for further explanations).

Using the shape of Figure 27(a) as an initial guess for problem (36), the optimized shapes for different values of $d_{\text {min }}$ are shown in Figure 28. As we could have expected, for relatively small values of $d_{\text {min }}$, the algorithm augments the size of thin members and, usually, reduces a bit the size of features whose thickness exceeds this value. In such cases, the shape does not change significantly and the final topology is the same with the initialization. However, when the value of $d_{\min }$ is such that several parts of the structure are penalized, then we can expect topological changes to occur. Initially, the size of the bars increases and then, since the compliance constraint is no more active, it is easier for the shape to change. Members can merge, which is usually beneficial for the minimum thickness constraint. We can see this process in Figure 29, where several intermediate iterations are depicted before arriving to the optimized shape of Figure 28(d).

Remark 8.1. One may wonder why the upper-right bar of the shape in Figure 27(b) does not disappear. This is an effect of the boundary condition for the level set function $\psi$ on the boundary $\partial D$ of the computational domain. Usually we do not enforce $\psi=0$ on $\partial \Omega \cap \partial D$ (see Figure 8) but rather we use a Neumann (or free) boundary condition. There are several reasons for this choice which is clearly a matter of modelization and debate. First, numerical experience shows that a Dirichlet boundary condition can lead to the appearance of spurious holes in the structure. Second, it may slow down the algorithm convergence, because the new optimizable boundary on $\partial D$ features high velocity (coming from the shape gradient) and thus the CFL condition for the advection of the level-set function becomes too strict. In any case, it is a purely numerical issue about the stability condition for the Hamilton-Jacobi equation. Usually, changing the boundary condition for $\psi$ does not change very much the resulting optimal shapes... 


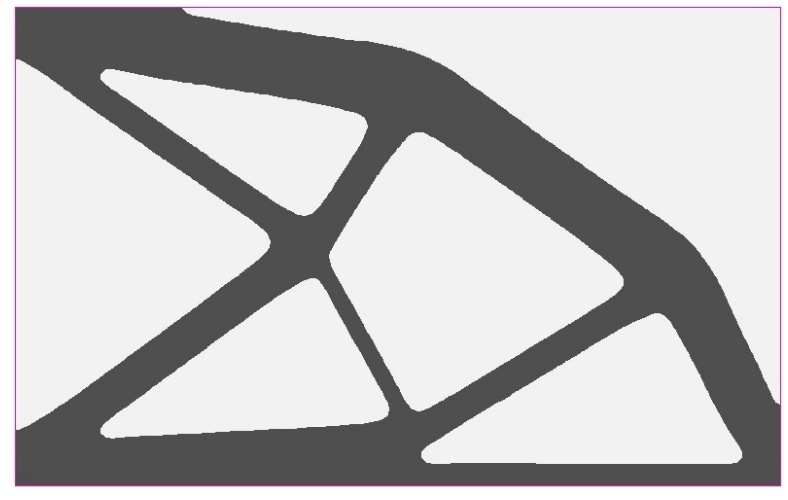

(a)

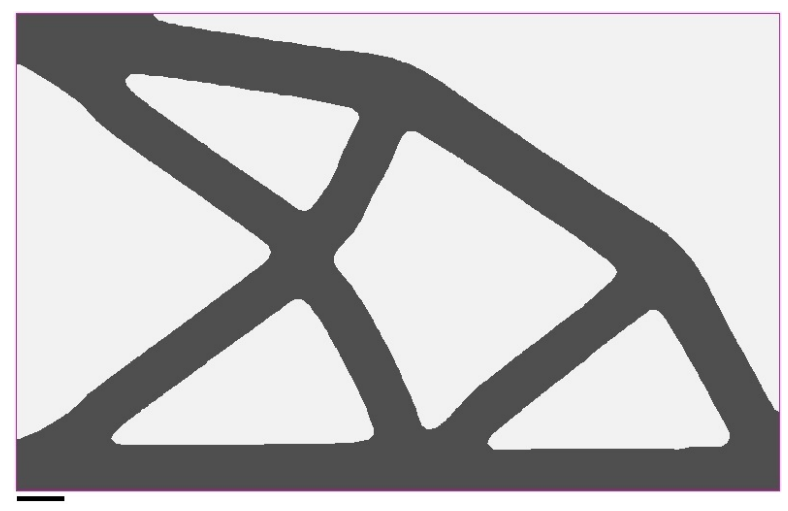

(c)

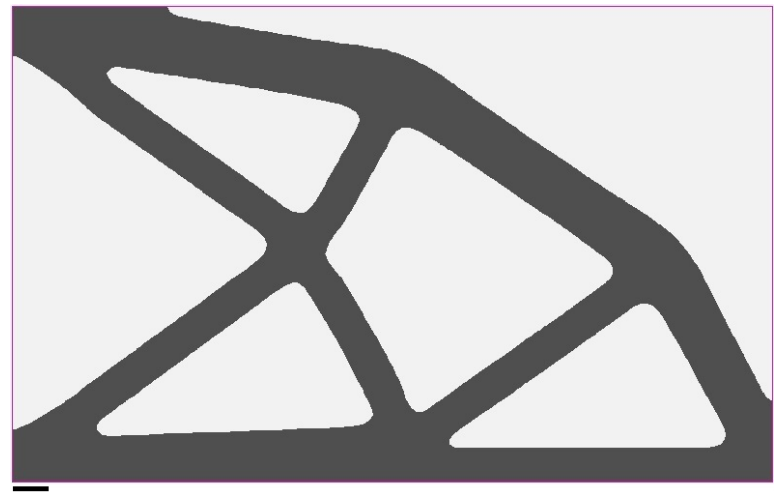

(b)

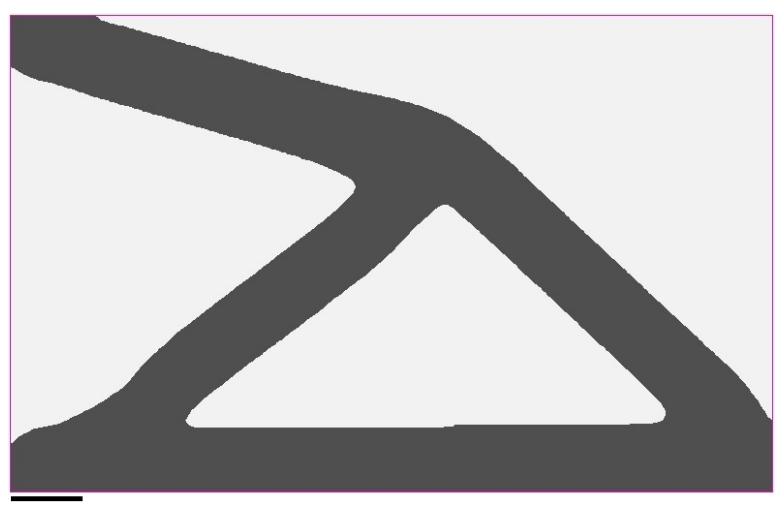

(d)

Figure 28: 2d cantilever; optimized shapes (a): without thickness constraint; imposing a minimum thickness limit (b): $d_{\min }=0.15 ;(\mathrm{c}): d_{\min }=0.20$ and $(\mathrm{d}): d_{\min }=0.30$. 


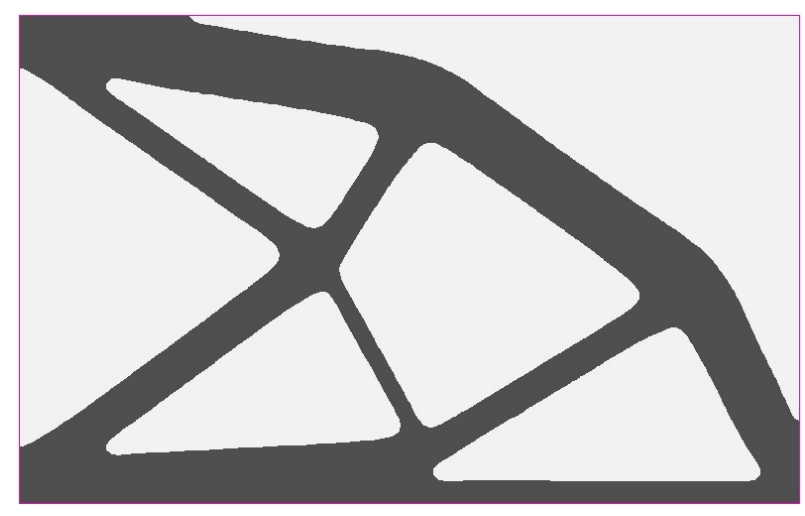

(a)

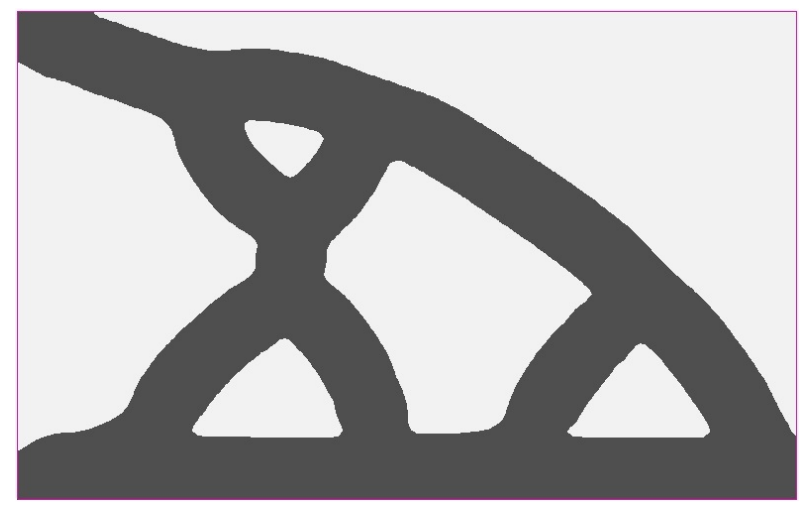

(c)

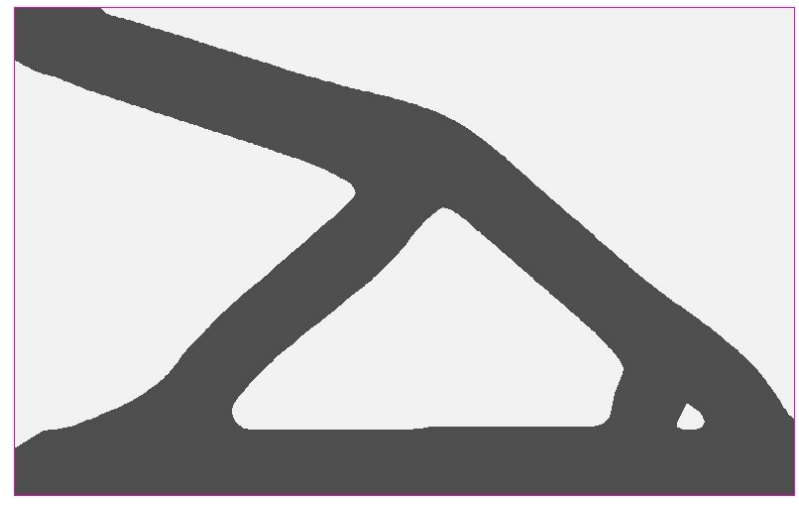

(e)

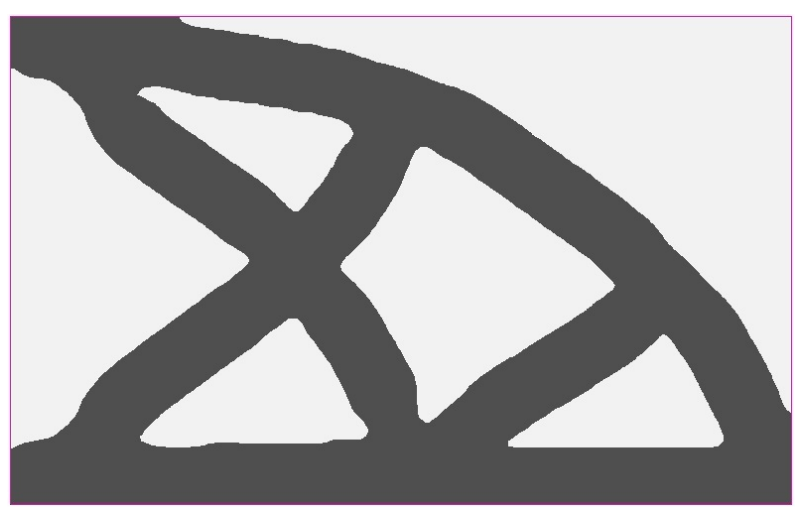

(b)

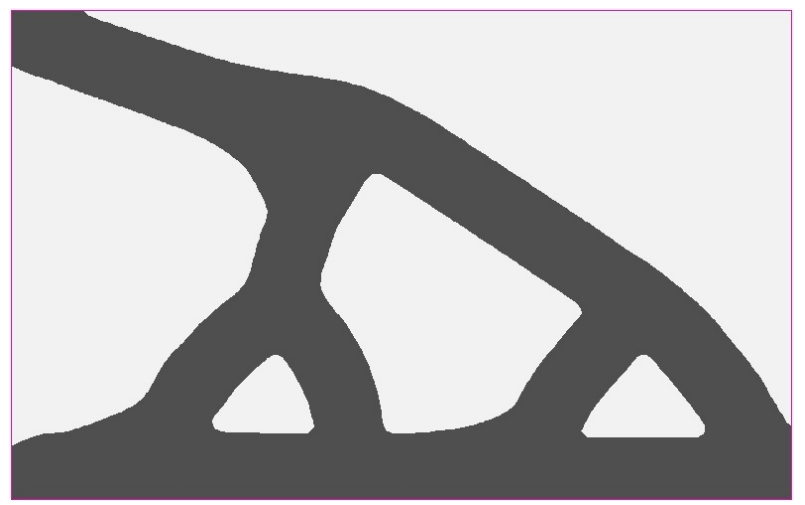

(d)

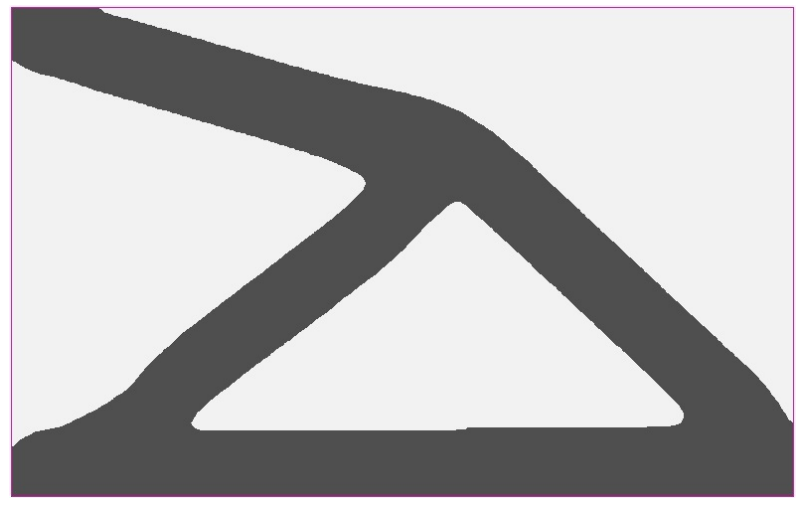

(f)

Figure 29: 2d cantilever; (a): initialization; iterations (b): 30, (c): 100, (d), 150, (e): 300; (f): optimized shape, imposing a minimum thickness limit $d_{\min }=0.30$. 
Table 4: 2d displacement inverter.

\begin{tabular}{|l|c|c|c|c|c|}
\hline & Without thickness restriction & $d_{\min }=0.05$ & $d_{\min }=0.06$ & $d_{\min }=0.07$ & $d_{\min }=0.08$ \\
\hline$u_{h}\left(x_{\text {tar }}\right)$ & -47.56 & -12.34 & -5.53 & -0.45 & 3.89 \\
\hline
\end{tabular}

except when a minimal thickness constraint is enforced, as in Figure 27(b). The upper-right bar cannot disappear because its tip is not a shape boundary ( $\psi$ is not zero) and thus is not advected by any shape gradient.

There are several remedies to avoid such artifacts in the case of minimal thickness constraints, but the best strategy is very likely case-dependent. For example, starting from the optimized shape of Figure 27(b), we change the boundary condition

$$
\psi(x)=0 \quad \forall x \in \partial \Omega \cap \partial D,
$$

and we continue the optimization process where now the upper-right bar tip can move. We perform only one iteration with this new Dirichlet boundary condition and we obtain the shape of Figure 30(a) where the upper-right bar has detached from the boundary $\partial D$. Therefore, we can again apply the usual Neumann boundary condition for $\psi$ (which is preferable for numerical stability reasons) and we continue the optimization algorithm for problem (36). The resulting "more acceptable" optimal shape, as well as intermediate iterations, are displayed on Figure 30. Note that, since the initialization is very far from an optimum, the optimization needs a lot of iterations to converge.

\subsubsection{2d displacement inverter}

The last example of this section concerns the design of a two-dimensional displacement inverter mechanism. The displacement of the upper-left and lower-left corners are fixed and we apply a unitary horizontal force at the middle of the left side (see Figure 31). We minimize the horizontal displacement $u_{h}$ at the middle point of its right side, denoted $x_{t a r}$. The optimization problem reads

$$
\begin{aligned}
\min _{\Omega \in \mathcal{U}_{a d}} & u_{h}\left(x_{\text {tar }}\right) \\
\text { s.t. } & \int_{\Omega} d x \leq V_{\text {max }} .
\end{aligned}
$$

Due to symmetry, only the upper half-domain is used for the analysis and is discretized by $160 \times 80$ elements. In all results here, $V_{\max }$ is set to $0.30|D|$. An initialization and its corresponding optimized shape are shown in Figure 32. As expected, we observe some very thin parts that act like hinges, a fact which was already recognized in [7], [41], [43]. The stiffness at these areas is artificially high due to erroneous finite element modelling [44], which increases the probability of failure of the actually fabricated shape [43]. Moreover, the scale of interest for such structures is so small, that the geometric uncertainty on the actually manufactured shape may have a significant impact [10], [43]. Consequently, designs containing thin features shall be avoided for such structures. One way to achieve this goal is to impose a minimum thickness constraint and solve the problem

$$
\begin{aligned}
\min _{\Omega \in \mathcal{U}_{a d}} & u_{h}\left(x_{t a r}\right) \\
\text { s.t. } & \int_{\Omega} d x \leq V_{\max }, \\
& P_{\operatorname{MinT}}(\Omega)=0 .
\end{aligned}
$$

The optimized shapes and their deformed configurations for different values of $d_{m i n}$ are shown in Figure 33. In Table 4, we display the final displacement for each case. As expected, increasing the minimum thickness limit $d_{\text {min }}$ leads to a less compliant structure and consequently to a greater displacement value. For $d_{\text {min }}=0.08$, we see that the displacment of the optimized shape has become positive, i.e. the structure no longer acts as a displacement inverter.

\subsection{Combination of thickness constraints}

There is no theoretical difficulty in combining the previously presented thickness constraints. Of course, adding more constraints in the optimization algorithm increases its complexity, as well as the possibility that it does not manage to find a feasible shape, either because it does not exist, or because the neccessary topological changes are not likely to take place. 


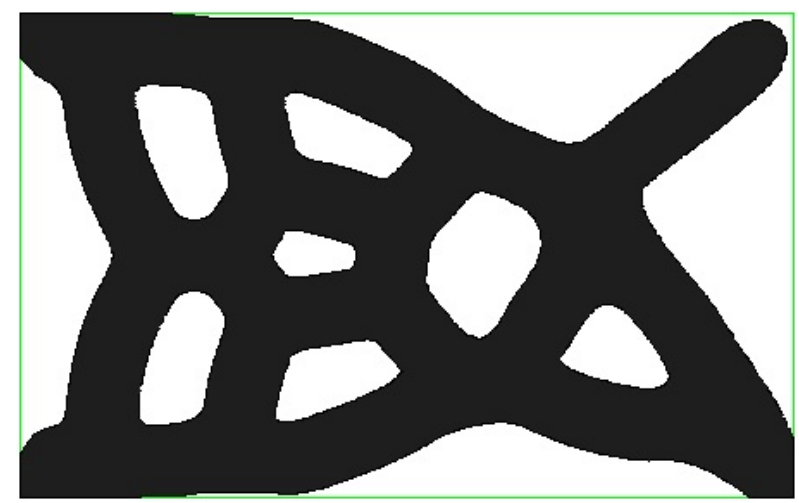

(a)

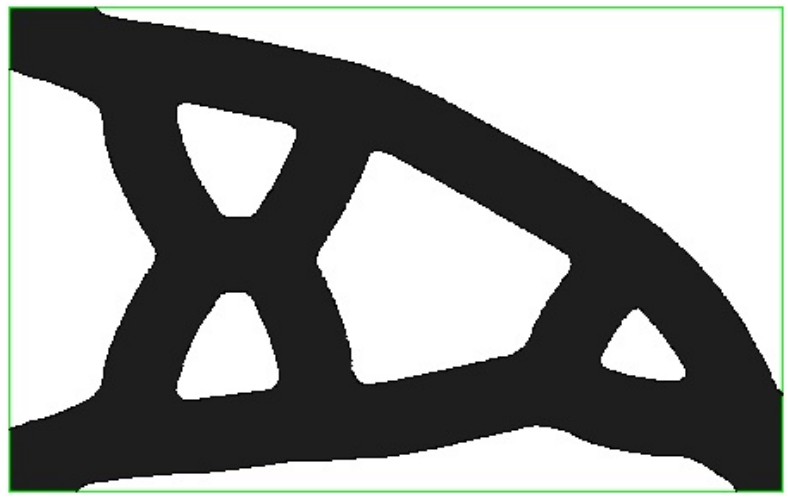

(c)

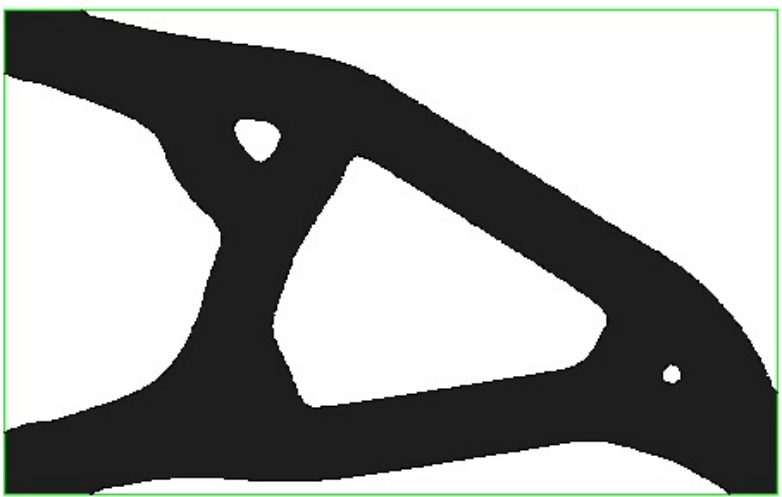

(e)

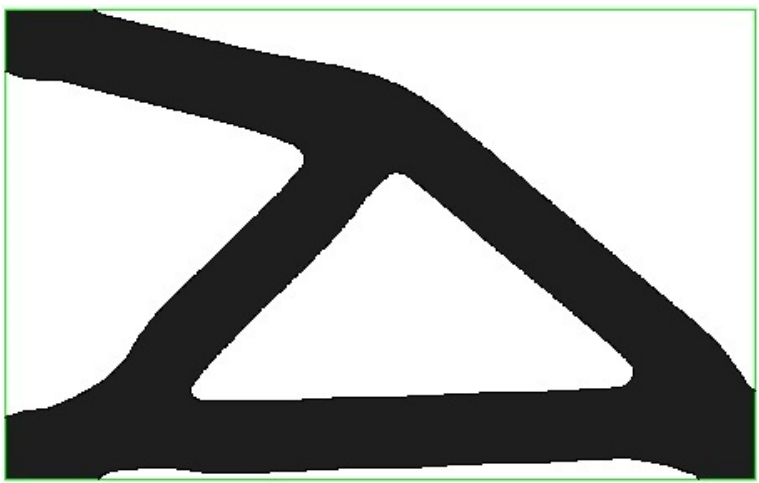

(g)

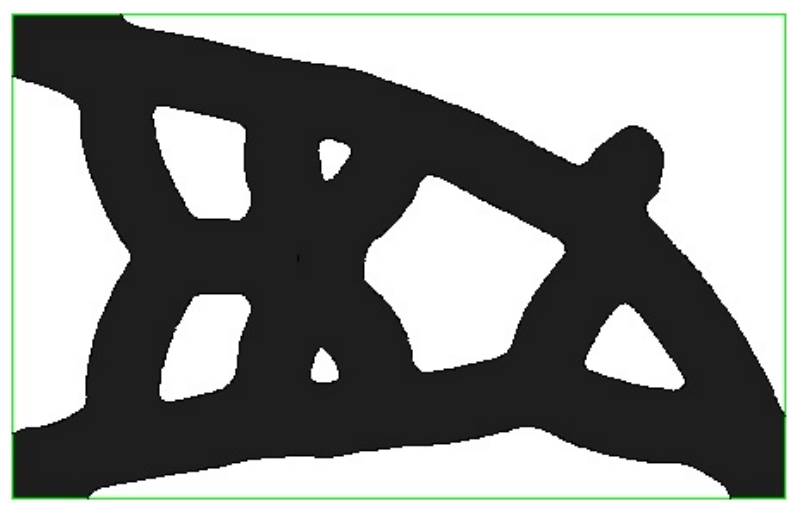

(b)

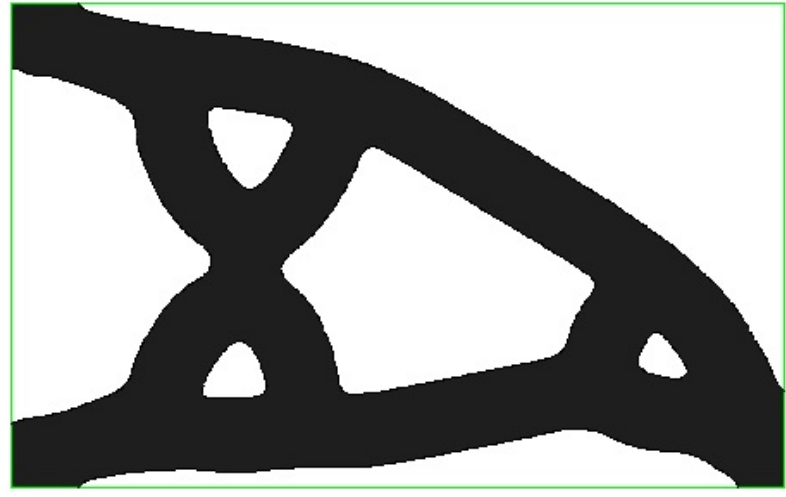

(d)

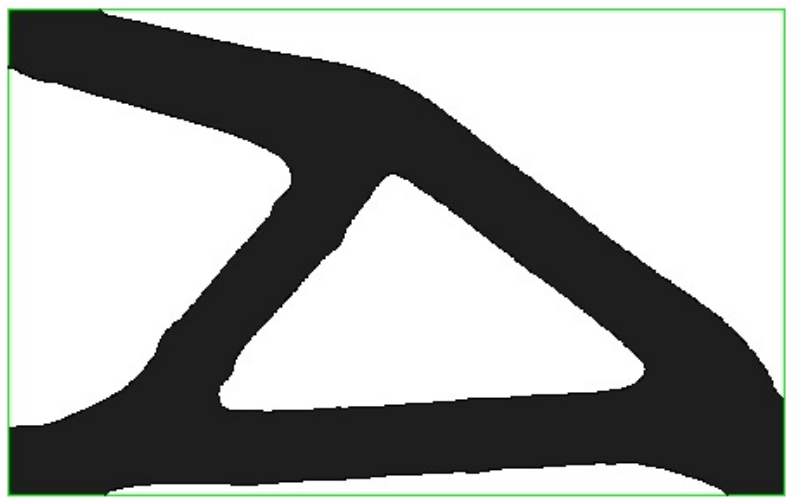

(f)

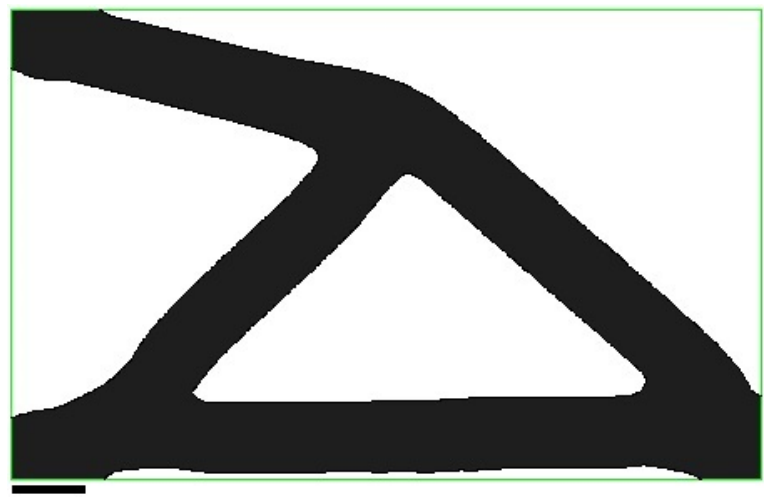

(h)

Figure 30: (a)-(h): Iterations 1,100,200,300,400,500,600,700 for the problem (36), for the $2 \mathrm{~d}$ cantilever, starting from the shape of Figure 27 (b). 


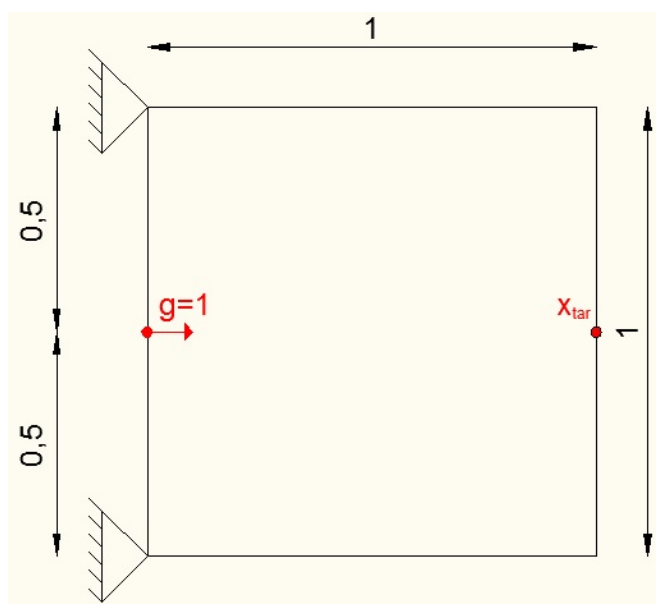

Figure 31: Boundary conditions for the displacement inverter mechanism.

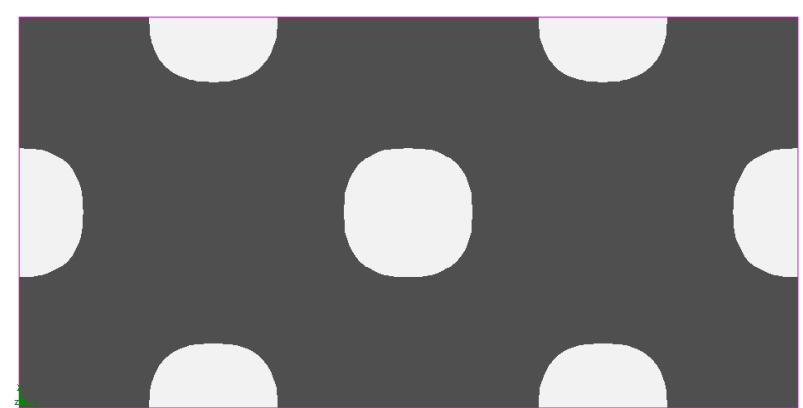

(a)

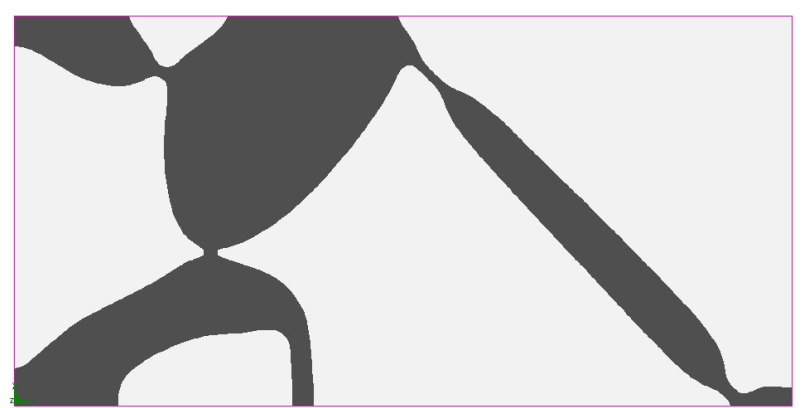

(b)

Figure 32: (a): Initialization and (b): optimized shape, without thickness limitations, for the displacement inverter mechanism. 

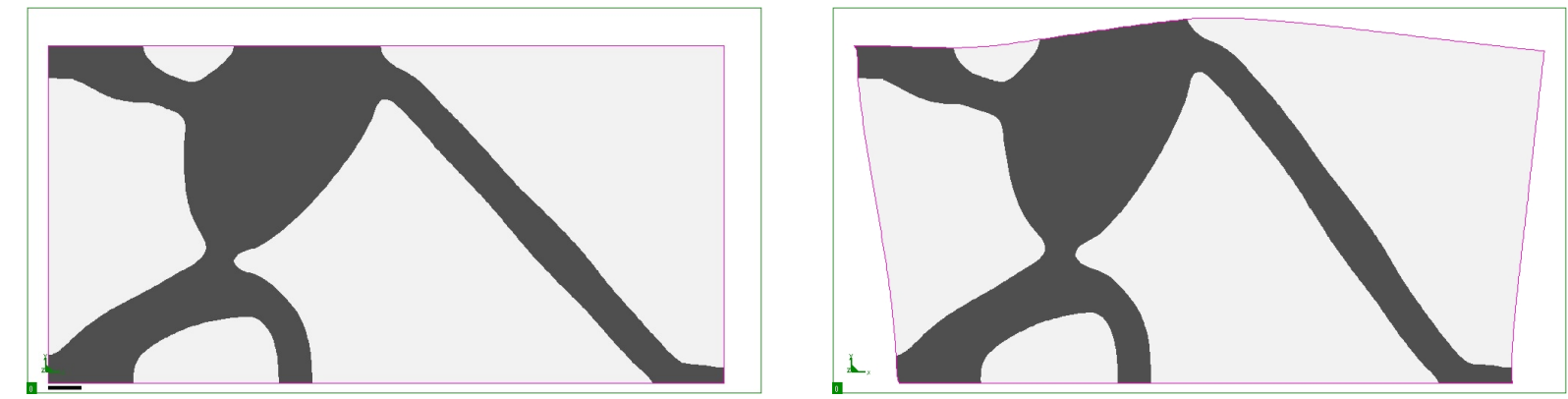

(a)
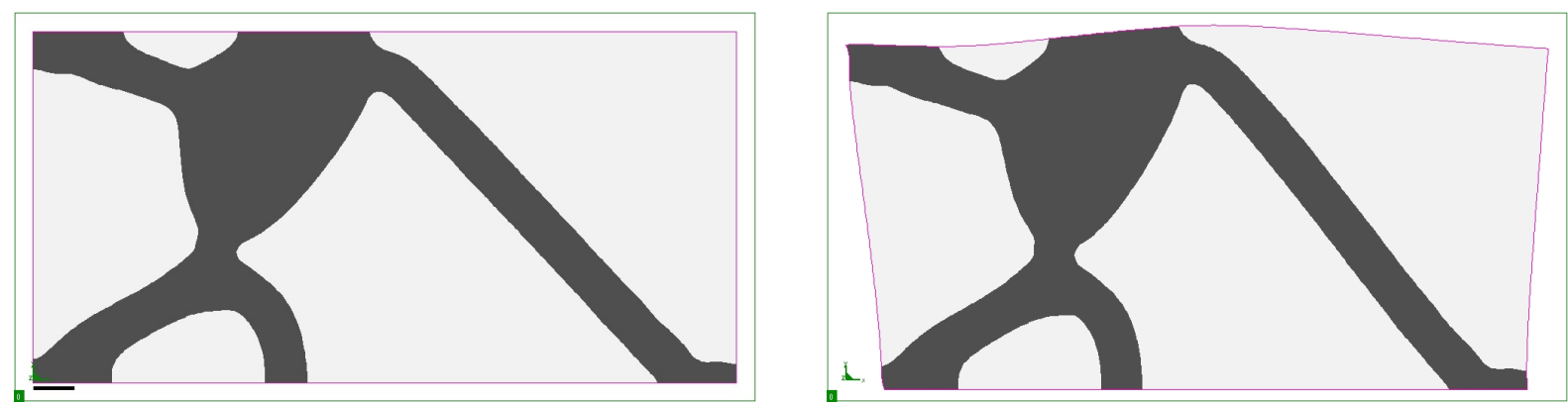

(b)
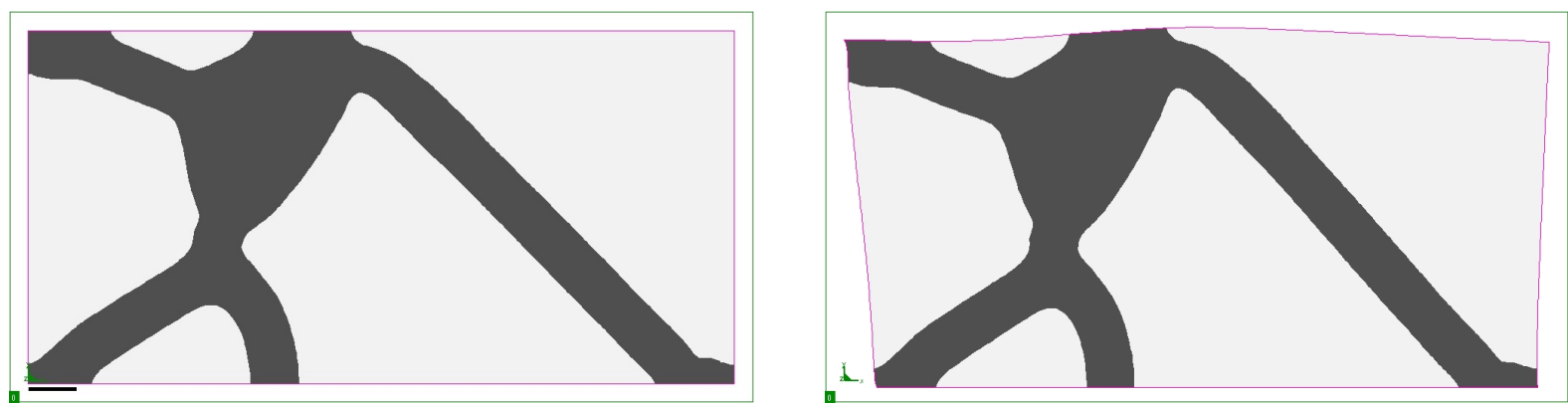

(c)
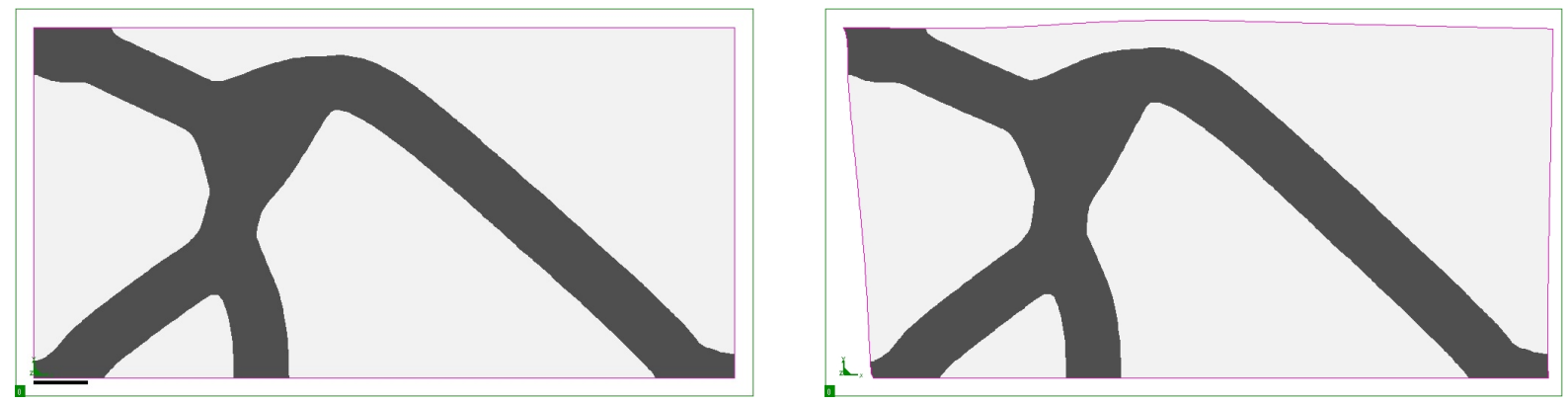

(d)

Figure 33: 2d displacement inverter; optimized shapes and deformed configurations imposing a minimum thickness limit (a): $d_{\min }=0.05 ;(\mathrm{b}): d_{\min }=0.06 ;(\mathrm{c}): d_{\min }=0.07 ;(\mathrm{d}): d_{\min }=0.08$. The boundary of the working domain $D$ is shown in magenta. A fixed frame is plotted in green to facilitate the comparison of the deformed shapes. 


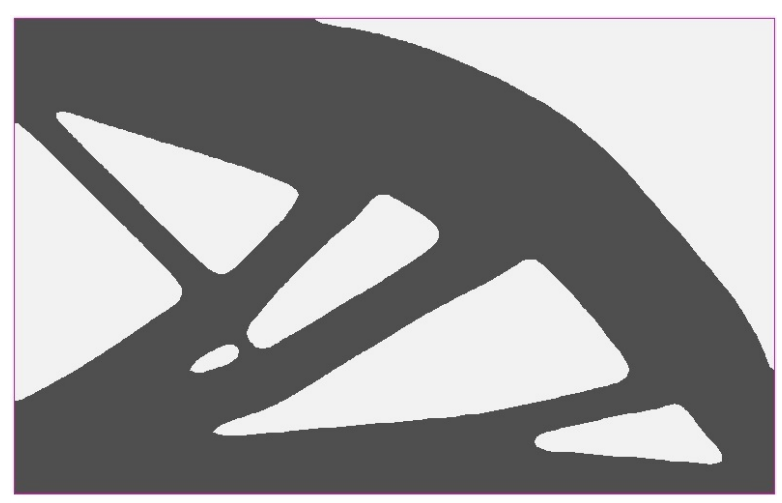

(a)

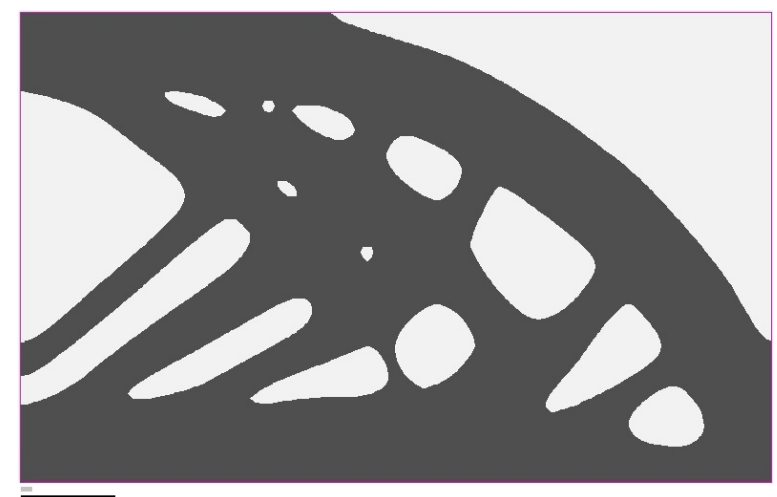

(c)

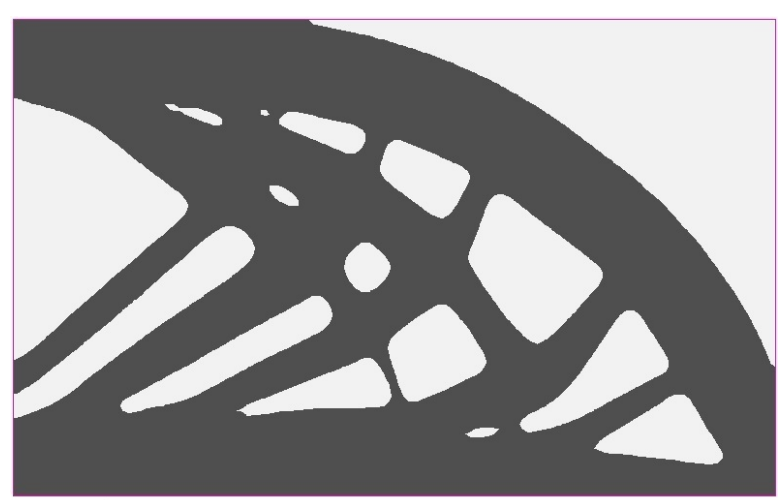

(b)

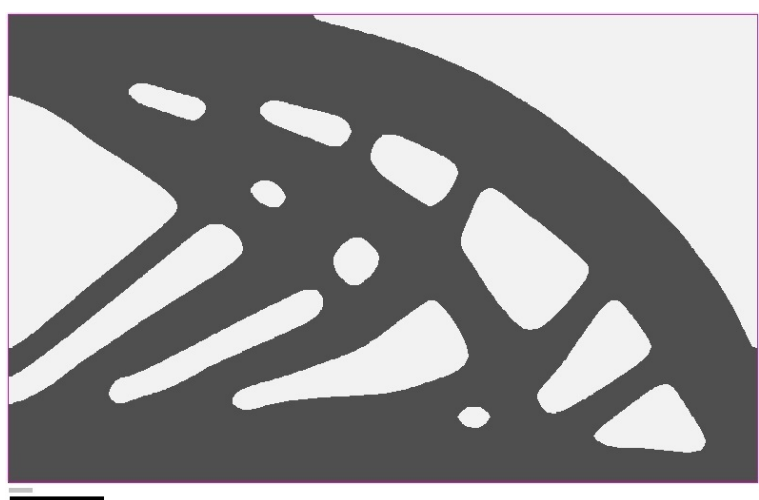

(d)

Figure 34: 2d cantilever; optimized shapes for (a): $g_{\max }^{1}=40$; (b): $g_{\max }^{1}=40$ and maximum thickness limit $d_{\max }=0.40 ;(\mathrm{c}): g_{\max }^{1}=40$, maximum thickness limit $d_{\max }=0.40$ and minimum members' distance limit $d_{m m d}=0.05 ;(\mathrm{d}): g_{\max }^{1}=40$, maximum thickness limit $d_{\max }=0.40$ and minimum members' distance limit $d_{m m d}=0.10$.

\subsubsection{Maximum thickness and minimum distance between members}

We consider once more the 2d cantilever of Figure 26(a). Starting with the initialization of Figure 26(b) and solving problem (34) for $g_{\max }^{1}=40$, we obtain the optimized shape of Figure 34(a). Starting again with the initial guess shape of Figure 26(b), adding a maximum thickness constraint and solving problem (35) for $d_{\max }=0.40$, we get the optimized shape of Figure 34(b), where we can observe the existence of small holes and closely spaced parts in the shape that we would like now to avoid. Starting from the shape of Figure 34(b), we add a constraint on the minimum distance between members to problem (35). The new optimization problem reads

$$
\begin{array}{ll}
\min _{\Omega} & \int_{\Omega} d x \\
\text { s.t. } & \int_{\partial \Omega} g \cdot u d s \leq g_{\max }^{1}, \\
& P_{1}(\Omega)=P_{\text {MaxT }}(\Omega) \leq g_{\max }^{2} \\
& P_{2}(\Omega)=P_{M M D}(\Omega)=0
\end{array}
$$

where $g_{\max }^{1}=40, d_{\max }=0.40$ and $d_{m m d}$ accounts for the minimum members' distance. The results for $d_{m m d}=0.05$ and $d_{m m d}=0.10$ are presented in figures $34(\mathrm{c})$ and $(\mathrm{d})$.

\subsubsection{Maximum and minimum thickness}

We consider again the MBB beam example of Figure 18. In Figure 20 we have shown the optimized shapes when a maximum thickness constraint is added to the original problem (34). We can see, for example for $d_{\max }=0.30$, the apparition of thin features in the shape that we would like now to avoid. Starting from the shape of Figure 20(a), we also add a minimum thickness constraint to problem (35). 
The new optimization problem reads

$$
\begin{array}{ll}
\min _{\Omega} & \int_{\Omega} d x \\
\text { s.t. } & \int_{\partial \Omega} g \cdot u d s \leq g_{\max }^{1} \\
& P_{1}(\Omega)=P_{\operatorname{Max} T}(\Omega) \leq g_{\max }^{2} \\
& P_{2}(\Omega)=P_{\operatorname{MinT}}(\Omega)=0
\end{array}
$$

where $g_{\max }^{1}=40, d_{\max }=0.30$ and $d_{\min }$ accounts for the minimum thickness limit. The results for $d_{\text {min }}=0.10$ and $d_{\text {min }}=0.15$ are presented in Figure 35 .

\section{Other formulations of thickness constraints}

In this section, we discuss some additional methods for thickness control. Some of them are inspired by formulations proposed by J. Guest et al. in [19], [23] in the framework of the SIMP method and we explore if the same logic can be applied in the context of the level-set method for shape and topology optimization.

\subsection{Maximum Thickness}

\subsubsection{Formulation of a constraint based on void percentage}

The initial concept of the maximum thickness constraint formulated in [19] is the following: defining at each point $x \in \Omega$ a ball of center $x$ and radius $r=d_{\max } / 2$,

$$
B(x, r)=\{y \in \Omega, \text { such that }|x-y| \leq r\},
$$

the pointwise constraint reads

$$
\int_{B(x, r) \cap D} \rho(y) d y<V_{B(x, r) \cap D},
$$

where $\rho(y)$ is the material density and $V_{B(x, r) \cap D}$ is the volume of the intersection of the ball $B(x, r)$ with the working domain $D$. The interpretation of the above constraint in the framework of a "classical" shape description would be

$$
\int_{B(x, r) \cap D} \chi_{\Omega}(y) d y<V_{B(x, r) \cap D}
$$

where $\chi_{\Omega}$ is the characteristic function of the shape $\Omega$.

Then, the authors of [19] propose to use another formulation instead of (41), that takes under consideration the volume of the void part in $B(x, r)$. The resulting pointwise constraint, again in a "classical" description, reads

$$
\int_{B(x, r) \cap D}\left(1-\chi_{\Omega}(y)\right) d y \geq V_{\min }
$$

where $V_{\min }>0$ is the minimum required volume of void part in $B(x, r)$. One proposal is to take $V_{\text {min }}=$ $\alpha_{\text {void }} V_{B(x, r) \cap D}$, where $\alpha_{\text {void }} \approx 5 \%$ is a small scalar parameter that defines the minimum percentage of void in any ball $B(x, r)$.

As in Section 4.4, we transform (43) into an integral constraint similar to (18). To simplify the notations, let us rewrite the pointwise constraint (43) in the general form

$$
g(\Omega, x) \leq g_{\max }(x), \quad \forall x \in \Omega,
$$

where $g(\Omega, x)=\int_{B(x, r) \cap D} \chi_{\Omega}(y) d y$ and $g_{\max }(x)=\int_{B(x, r) \cap D} d y-V_{\min }$. The right hand side of inequality (44) depends on the space variable $x$ because the volume of $B(x, r) \cap D$ is changing when $x$ approaches the boundary of the working domain $D$. Finally, introducing a regularization function $f$ similar to that in Figure 5, the global constraint reads

$$
P_{\operatorname{Max} T}(\Omega)=\left(\frac{\int_{\Omega} f(g) g(\Omega, x)^{2} d x}{\int_{\Omega} f(g) g_{\max }(x)^{2} d x}\right) \leq 1 .
$$

The shape derivative of (45) has been calculated and various numerical test cases have been performed in [29] (pp. 125-127). The next section makes comparisons with our formulation in Section 4.4. 


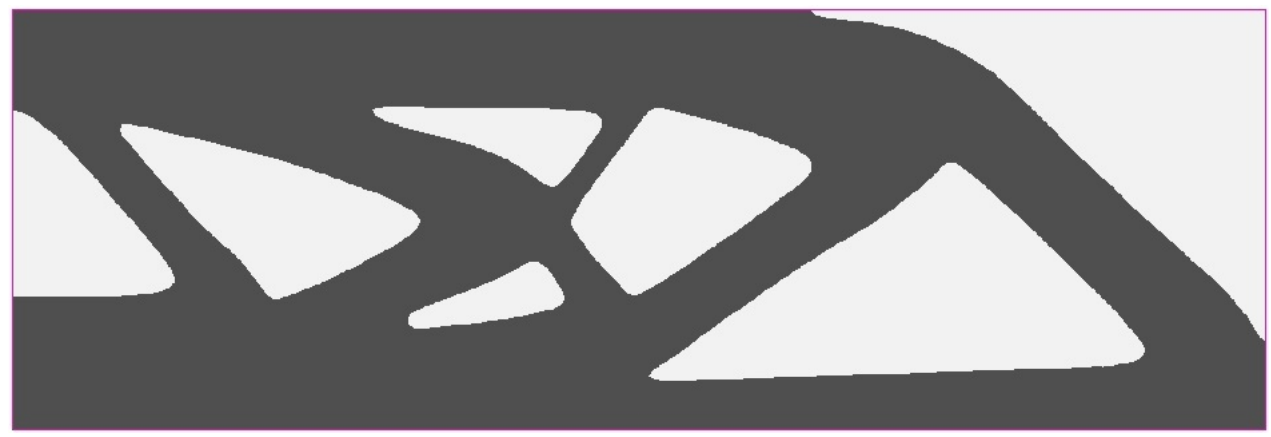

(a)

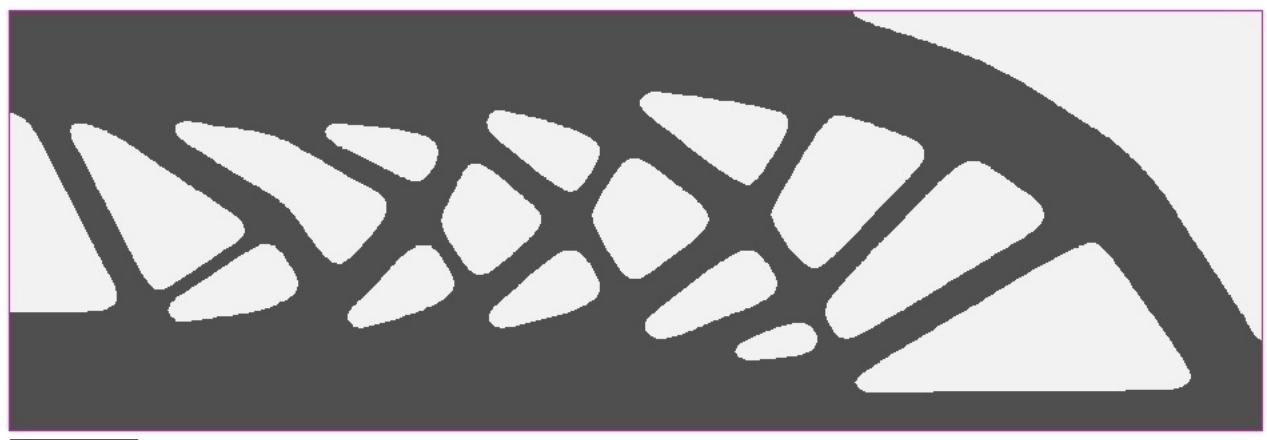

(b)

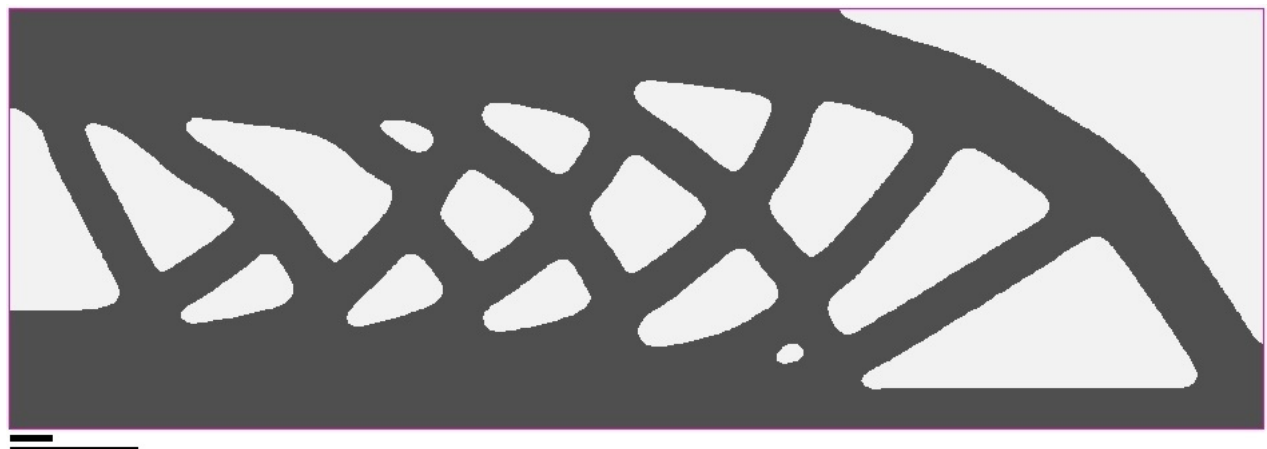

(c)

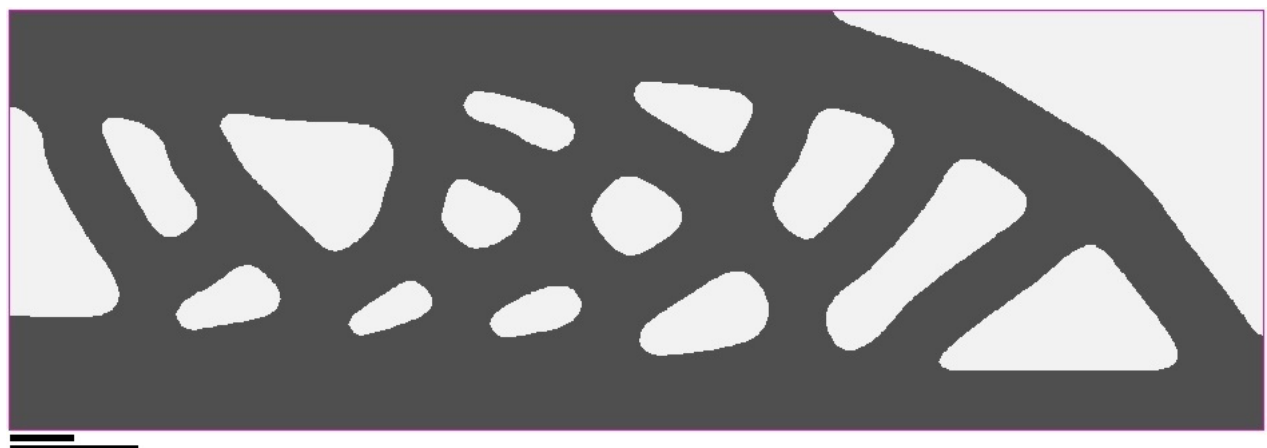

(d)

Figure 35: 2d MBB beam; optimized shapes for (a): $g_{\max }^{1}=40 ;(\mathrm{b}): g_{\max }^{1}=40$ and maximum thickness limit $d_{\max }=0.30 ;(\mathrm{c}): g_{\max }^{1}=40$, maximum thickness limit $d_{\max }=0.30$ and minimum thickness limit $d_{\min }=0.10 ;(\mathrm{d}): g_{\max }^{1}=40$, maximum thickness limit $d_{\max }=0.30$ and minimum thickness limit $d_{\min }=0.15$. 


\subsubsection{Comparison with the formulation of Section 4.4}

We begin with the general formulation (42) and prove its equivalence with our formulation (12) based on the signed distance function. When inequality (12) is satisfied, there exists no point whose distance to the boundary is greater than $d_{\max } / 2$ and thus there is no point which is the center of a ball of radius greater than $d_{\max } / 2$, fully contained in the shape. Inequality (42) is strict, i.e. the allowed inscribed circle must be of radius less than $d_{\max } / 2$. Satisfying inequality (42) means that no inscribed circle of radius greater or equal to $d_{\max } / 2$ exists and thus no point is at a distance greater than $d_{\max } / 2$ from the boundary, so inequality (12) is also satisfied.

Let us pass now to constraint (43) which we view as a relaxation of (42), numerically easier to implement. Indeed (42) is always true if the inequality is large, so that it is a constraint only when the inequality is strict. Numerically, enforcing a strict inequality constraint is not easy to handle. A drawback of formulation (43) is that the maximum allowed thickness is reduced from its initial value $d_{\text {max }}$, depending on the choice of the parameter $\alpha_{\text {void }}$ in $V_{\min }$. However, a relative advantage of (43) lies in the treatment of small holes which artificially decreases the thickness, as explained in Section 4.5. Indeed, if $\alpha_{\text {void }}$ is not too small, then very small holes (like in part (a) of Figure 3) would not anymore reduce the value of $P_{\operatorname{MaxT}}(\Omega)$ up to the point that the maximum thickness constraint is satisfied. Nevertheless, we expect that small values of $\alpha_{\text {void }}$ would lead to the same unstability phenomenon as described in Section 4.5. The interested reader can find in [29] (pp. 126-127) numerical results using (45) as maximum thickness functional and some comparisons with our proposed constraint (18). In our experience, formulation (45) is numerically unstable for small values of $\alpha_{\text {void }} \approx 0.01$, due to the creation and disappearance of small holes (as discussed in Section 4.5). For larger values of $\alpha_{\text {void }} \approx 0.05$ or 0.1 ), which of course imply a modification of the initially imposed maximum thickness limit $d_{\max }$, the method is stable. For a $2 \mathrm{~d}$ cantilever test case, the formulation based on (18) provided the lightest optimized shape (see Table 3.7 in $[29])$.

The computational cost increases with mesh refinement for all methods we have presented for the maximum thickness. Concerning the methods of Section 4.4, the "true" and "Jacobian-free" formulas for the shape derivative require to integrate alongs ray (of fixed length) which takes more time as the mesh size decreases. The "approximate" formula requires to solve the regularization (29), i.e., solving a linear system, the size of which increases as the mesh size decreases. However, the increase in computational time for the void percentage formulation (43) is much more dramatic since evaluating $g(\Omega, x)$ in (44) is a convolution (computing an integral on the ball $B(x, r)$ ) which is a costly operation, all the more for large values of $d_{\max }$.

\subsection{Minimum Thickness}

\subsubsection{Formulation of a constraint based on the skeleton}

One may wonder if it is possible to formulate a minimum thickness constraint in the form of (12) instead of using the notion of offset sets, as in (13). Of course such a new constraint would not hold for points $x \in \partial \Omega$ (where we always have $d_{\Omega}(x)=0$ ) and we have to decide for which points it should be imposed. A reasonable idea (proposed and tested in [24]) is to test the signed distance function at the points that belong to the skeleton $\Sigma$. In other words, a possible constraint would be

$$
d_{\Omega}(x) \leq-d_{\min } / 2, \quad \forall x \in \Sigma .
$$

One advantage of (46) is that it gives a local definition of the thickness of the structure which is understood as the (absolute) value of the distance function on the skeleton. On the other hand, the constraint (46) does not always give a satisfying definition of the minimum feature size. Consider, for example, a rectangular shape with rounded corners, as in Figure 36. It is a very smooth shape. Its skeleton is close to the boundary in the vicinity of the rounded corners. Actually, the distance from the skeleton to the boundary is exactly the radius of curvature of the rounded corners. Therefore, although the signed distance function $d_{\Omega}$ on the horizontal part of the skeleton is exactly equal to the half-thickness of the rectangle (as desired), it is decreasing on the oblique parts of the skeleton (which are oriented with \pm 45 degrees angles with respect to the rectangle axis) up to very small values close to the corners. On the contrary, our definition of thickness for this rectangle, based on offset sets and (13), is conformed to the geometric intuition. Note that there are other geometries for which neither our formulation (13), nor (46), give a meaningful definition of minimal thickness. For example, because of its small bump of size $\epsilon$, the perturbed disk on the right of Figure 37 has a minimal thickness of order $\epsilon$, much smaller than its diameter. This shows that no (known) formulation of minimal thickness constraint is perfect... 


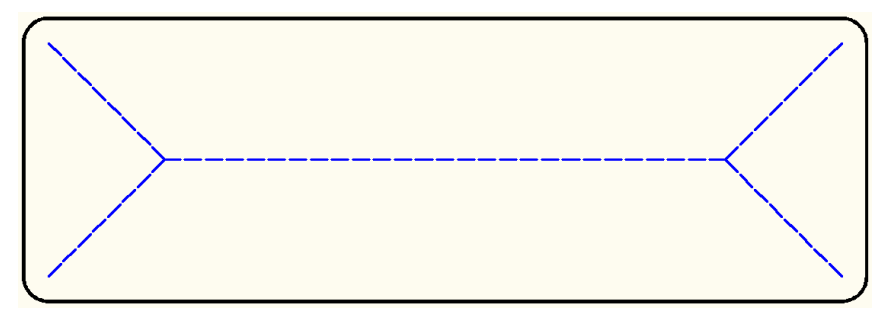

Figure 36: Skeleton (in blue) of a smooth shape.
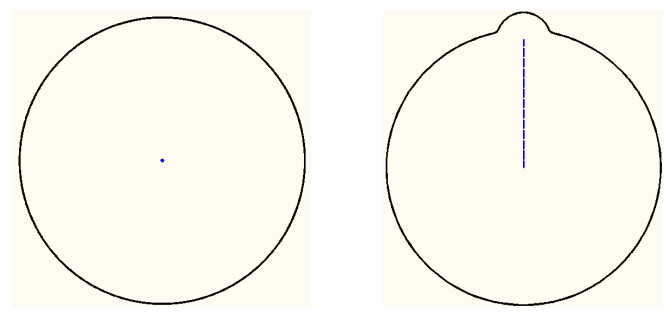

Figure 37: Discontinuity of the skeleton (in blue) for a perturbed disk.

Even if it were possible to eliminate these oblique parts of the skeleton in the proposed constraint (46) and thus remove the above difficulty, computing the derivative of (46) is a challenging problem, as we now explain. Indeed, we have no information about how the skeleton of the shape changes with respect to arbitrary shape variations. In [24], the authors proposed to neglect the term of the shape derivative that corresponds to the change of the skeleton, while in [51] the authors did not consider this effect in the derivation with respect to the density field. Furthermore, the expression for $d_{\Omega}^{\prime}(\theta)$ is not straightforward at points on the skeleton, since it depends on the choice of $\theta$ (see Remark 2.6 in [4]). If one considers smooth shapes of class $C^{2}$, the skeleton varies continuously with respect to shape variations (we don't know of any formula for its shape derivative). However the continuity of the skeleton is not true for some specific perturbations, as illustrated by the two following examples. In Figure 37 we consider a disk with a small bump which has a radial line as its skeleton, in contrast with the case of a perfect disk which has one single point (its center) as skeleton. Note that the bump is a small perturbations of the shape in the continuous norm but not in the $W^{1, \infty}$-norm, as usual in the framework of Hadamard method for shape derivative (however, from a practical point of view, this counter-example is sufficiently illuminating). (This small bump is not a small perturbation in the Hadamard framework but it is a typical perturbation in the context of the so-called topological gradient [46].) In Figure 38 we consider a rectangle which is perturbed by transforming its upper side in a roof making a small angle with the horizontal direction. In this last counter-example the perturbation is small in the $W^{1, \infty}$-norm but the shape is just Lipschitz-smooth since it has corners. Therefore, these differentiability issues are another reason why we prefer our formulation (13), versus (46), for minimum thickness constraint (see [29], pp. 120).

\subsubsection{Energy functionals}

Instead of formulating a constraint, another idea is to add a penalization term, or energy functional, to the objective function, in the hope that it will hinder the creation of thin features in the structure. This approach has been followed in [11], [12], [28]. However, instead of favoring specific patterns and shapes,

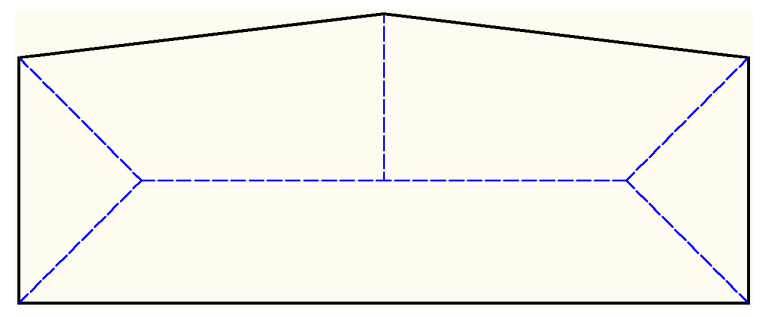

Figure 38: Discontinuity of the skeleton (in blue) for a perturbed rectangle (compare to Figure 36). 
as in these previous works, we suggest an energy functional involving the signed distance function. In addition, we define the functional so that it has no impact on features with a thickness exceeding the desired limit. We thus introduce the following energy functional

$$
E(\Omega)=-\int_{\Omega} d_{\Omega}(x)^{2}\left[\left(d_{\Omega}(x)+d_{\min } / 2\right)^{+}\right]^{2} d x .
$$

Instead of problem (36), where a minimum thickness constraint is formulated, a weighted sum of the volume and of the above energy functional is considered

$$
\begin{aligned}
\min _{\Omega \in \mathcal{U}_{a d}} & \int_{\Omega} d x+\ell_{E} E(\Omega) \\
\text { s.t. } & \int_{\partial \Omega} g \cdot u d s \leq g_{\text {max }}^{1},
\end{aligned}
$$

where $\ell_{E} \geq 0$ is the weight coefficient for the energy functional. A satisfying scaling between the volume and the thickness energy is achieved by choosing $\ell_{E}=V\left(\Omega^{0}\right) / E\left(\Omega^{0}\right)$, where $\Omega^{0}$ stands here for the optimized shape without the minimum thickness constraint.

Adding energy (47) since the beginning of the optimization algorithm requires some more attention. The choice of the weight coefficient $\ell_{E}$ plays a major role in the efficiency of the algorithm. If we adopt the same scaling as before, it is highly possible that very slight changes in the initial topology will occur. Instead we can choose to start with a smaller value of $\ell_{E}$ and increase it during the optimization.

Energy functionals for the control of minimum thickness have been discussed in details in [29] (pp. 98-120). In particular, we studied the behaviour of (47) when topological changes occur and we proposed some alternative formulations.

The numerical calculations of (47) and its shape derivative, are much easier compared to those for the penalty functional $P_{\text {MinT }}(\Omega)$. Moreover, the energy functional (47) is less sensitive to the mesh size than $P_{M i n T}(\Omega)$, which requires a fine enough mesh in order to be accurately approximated. However, contrary to $P_{\text {MinT }}(\Omega), E(\Omega)$ does not actually impose a precise value of features' size, but instead penalizes too thin features. Furthermore, the resulting optimal shape strongly depends on the choice of the weight coefficient $\ell_{E}$. We suggest that, when there is no precise minimum feature size to be imposed, using the energy functional $E(\Omega)$ can be a good alternative to $P_{M i n T}(\Omega)$.

\subsubsection{Design and physical variables}

In [23], Guest et al. proposed a new idea to tackle the minimum thickness specification. Instead of formulating a constraint, they integrated the constraint in the optimization variables. As a first step, the distinction between "design" and "physical" variables is done. Design variables are the optimization variables and they determine the physical variables, on which the material properties depend. The physical variables are chosen as usual to be the densities of the elements of the mesh. In [23] the SIMP method was chosen to define the material properties in terms of the element densities.

The physical and the design variables are linked as follows. For every element $e$ of the mesh we define a domain of influence which is the ball $B\left(e, r_{\min }\right)$ of radius $r_{\min }=d_{\min } / 2$ centered at the center of the element (see Figure 39). At each node $j$ of the mesh, design variables $\rho_{j}$ are defined. The values of $\rho_{j}$ in the influence domain of each element $e$ determine the value of its physical density $\rho^{e}$. This is done in two steps. First, a weighted average of the design variables, also called "nodal volume fraction", is calculated in $B\left(e, r_{\min }\right)$ as

$$
\mu^{e}=\frac{\sum_{j \in S_{e}} \rho_{j} w\left(\left|x_{j}-\bar{x}^{e}\right|\right)}{\sum_{j \in S_{e}} w\left(\left|x_{j}-\bar{x}^{e}\right|\right)}
$$

where $S_{e}$ is the set of nodes belonging to $B\left(e, r_{\min }\right)$ (the nodes in green color for the element $e$ in Figure 39 ) and $w$ is a weighting function that depends on the distance between the position $x_{j}$ of node $j$ and the center $\bar{x}^{e}$ of the element $e$. Then, the element density is calculated as a Heaviside function such that

$$
\rho^{e}=\left\{\begin{array}{cc}
1 & \text { if } \quad \mu^{e}>\rho^{\min } \\
\rho_{\min }^{e} & \text { if } \quad \mu^{e}=\rho^{\text {min }}
\end{array}\right.
$$

where $\rho^{\text {min }}$ and $\rho_{\text {min }}^{e}$ are the minimum allowed densities for the nodal and the element variables, in order to avoid singularity of the stiffness matrix. In numerical practice, a regularized version of (49) is used.

The formation of features respecting some notion of minimum thickness is inherent in the formulation (49) of the element density. A great benefit of the method is its simplicity and ease of use at any step 


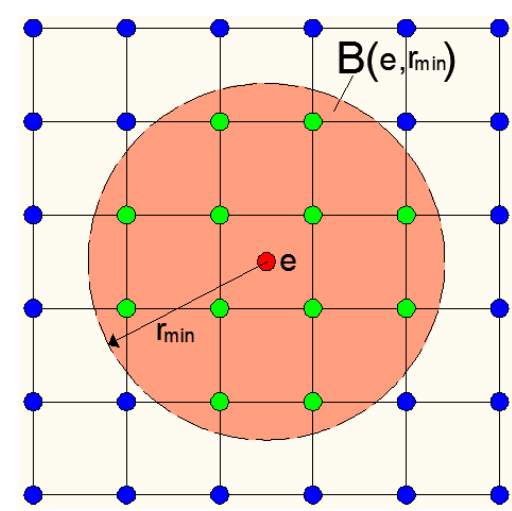

Figure 39: Influence area of an element $e$.

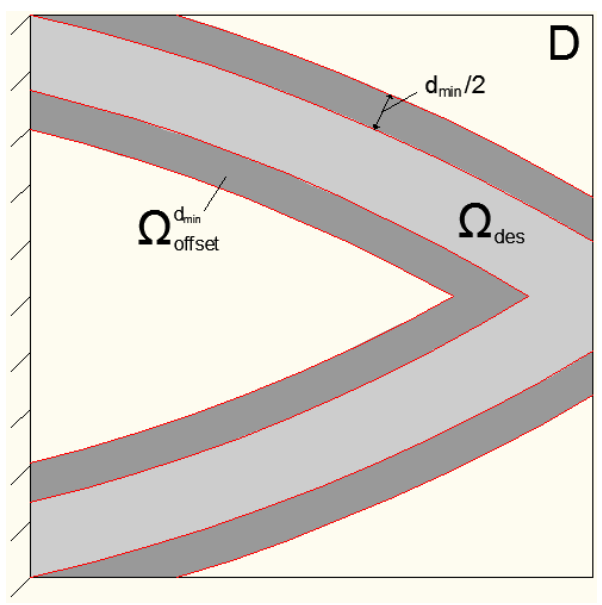

(a)

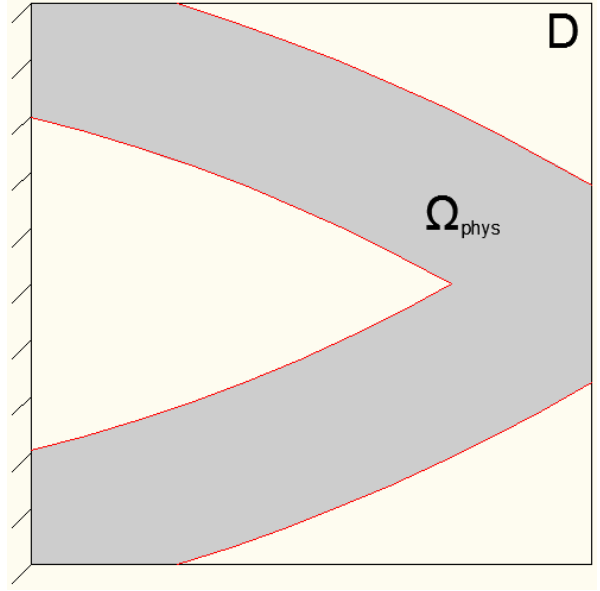

(b)

Figure 40: (a): Design $\Omega_{\text {des }}$ and offset $\Omega_{\text {off set }}^{d_{\min }}$ shapes ; (b): physical shape $\Omega_{\text {phys }}$.

of the optimization algorithm. Of course, it is expected that the algorithm is quite sensitive to the regularization parameters of (49) and to local minima. In [22] some modifications of the method of moving asymptotes (MMA) are proposed in order to circumvent some of these inconveniences.

\subsubsection{Interpretation in the context of geometric optimization}

We adapt the main concept of the method of [22], that is the distinction between design and physical variables, in the framework of geometric shape optimization. Since our optimization variable is the shape itself, we introduce the notions of "design shape" and "physical shape". We optimize the design shape in order to minimize an objective function that depends on the physical shape, which is the real shape. More precisely, denoting $\Omega_{\text {des }}$ the design shape, we define the physical shape $\Omega_{p h y s}$ by

$$
\Omega_{\text {phys }}=\Omega_{\text {des }} \cup \Omega_{\text {offset }}^{d_{\min }},
$$

where

$$
\Omega_{\text {offset }}^{d_{\min }}=\left\{x \in D \text { such that } d_{\Omega_{\text {des }}}(x) \leq d_{\text {min }} / 2\right\}
$$

is the set of points lying up to a distance $d_{m i n} / 2$ from the boundary $\partial \Omega_{\text {des }}$ (see Figure 40 ). A level-set function $\psi_{\text {phys }}(x)$ corresponding to the physical shape can easily be built in two ways. Either by creating $d_{\Omega_{\text {des }}}$, the signed distance function to the design shape, and setting $\psi_{\text {phys }}(x)=d_{\Omega_{\text {des }}}(x)-d_{\text {min }} / 2$, of by advecting the level-set function $\psi_{\text {des }}$ that describes the design domain up to a distance $d_{\text {min }} / 2$.

The general type of a shape optimization problem under a minimum thickness constraint now reads

$$
\inf _{\Omega_{\text {des }}} J\left(\Omega_{\text {phys }}\right),
$$




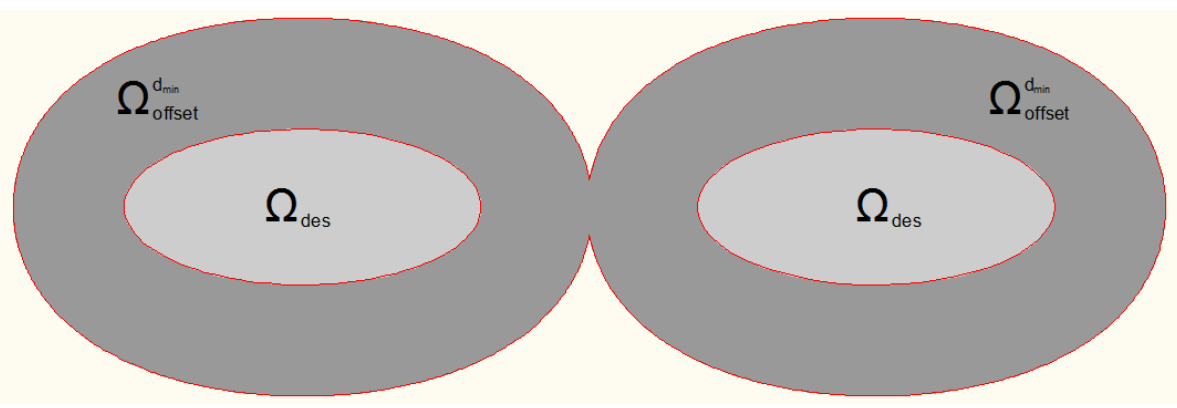

Figure 41: Formation of thin members using projection methods.

where the constraint does not appear explicitly, but has been incorporated into the relation (50) connecting the physical and the design shape. To apply a gradient-based algorithm to (51) one has to compute the shape derivative of $J\left(\Omega_{\text {phys }}\right)$. This is not straightforward, since the advection velocity is now defined on the boundary of the design domain $\Omega_{\text {des }}$. In other words, we need to find how changes in the domain $\Omega_{\text {des }}$ will affect $\Omega_{\text {phys }}$ so as to decrease the objective function.

In [29] (pp. 130-131) the shape derivative of $J\left(\Omega_{\text {phys }}\right)$ is computed but its formula is neither simple nor fully explicit. Furthermore, it is a valid computation under the assumption that the design and physical shapes are related by a Lipschitz diffeomorphism, i.e., that $d_{\min }$ is small enough and $\Omega_{\text {des }}$ is smooth enough. Due to the complexity of this analysis, we do not recommend this approach for minimum thickness constraint and we prefer that in Section 4.4.

Remark 9.1. A formula for the shape derivative of $J\left(\Omega_{\text {phys }}\right)$ is proposed in [10] but it is not a rigorous result and merely an approximation of the true derivative. Let us explain their result. Assume that the design and physical shapes are related by a diffeomorphism, namely $\Omega_{\text {phys }}=\left(\mathrm{Id}+\theta^{*}\right) \Omega_{\text {des }}$ for some vector field $\theta^{*}$ (cf. the notations of Section 2.2). In other words, to any point $x \in \Omega_{\text {des }}$ corresponds a point $X=\left(x+\theta^{*}(x)\right), X \in \Omega_{\text {phys }}$. The authors of [10] claimed that a descent direction for $\Omega_{\text {des }}$ can be obtained by

$$
v(x)=V(X),
$$

where $V(X)$ is the shape derivative on the physical domain $\Omega_{\text {phys }}$. Unfortunately, this analysis is not correct, since it neglects the fact that the diffeomorphism $\theta^{*}$ is shape dependent. For example, one possible choice of $\theta^{*}$ is

$$
\theta^{*}(x)=\left(d_{\min } / 2\right) n(x), \quad \forall x \in \partial \Omega_{\text {des }},
$$

where $n(x)$ is the exterior normal vector to $\partial \Omega_{\text {des }}$. Therefore, relation (52) does not necessarily guarantee a descent direction.

Despite its simplicity (the minimum thickness constraint has seemingly disappeared and is hidden in the link between design and physical variables), two major drawbacks seem to appear in projection methods. First, the method guarantees a minimum feature size only if the design domain is connected. In Figure 41, the design domain is composed by two disconnected parts and the physical domain contains a thin feature in the middle. The creation of such features will be promoted if this is beneficial for the objective function. For example, we believe that this is the case for the inverter mechanisms in [22], where the appearance of hinges is not prevented. Second, the use of projection methods presents several difficulties when several geometric specifications are simultaneously imposed (e.g. combining minimum and maximum thickness, minimum members' distance and molding direction constraints). Indeed, it is not clear how to relate the design and the physical variables in such a case and if such a definition would be efficient inside a standard optimization algorithm. We believe that, in the case of several geometric constraints, it is preferable to write each constraint separately and to use a constrained optimization algorithm.

Acknowledgments The authors acknowledge fruitful discussions and helpful remarks from Marc Albertelli (Renault) and Charles Dapogny (LJLL Paris VI - Renault). This work has been supported by the RODIN project (FUI AAP 13). G. Allaire is a member of the DEFI project at INRIA Saclay Ile-de-France. 


\section{References}

[1] O. Alexandrov and F. Santosa. A topology-preserving level set method for shape optimization. Journal of Computational Physics, 204(1):121-130, 2005.

[2] G. Allaire. Shape optimization by the homogenization method, volume 146 of Applied Mathematical Sciences. Springer-Verlag, New York, 2002.

[3] G. Allaire. Conception optimale de structures, volume 58 of Mathématiques 83 Applications (Berlin). Springer-Verlag, Berlin, 2007.

[4] G. Allaire, C. Dapogny, G. Delgado, and G. Michailidis. Mutli-phase structural optimization via a level-set method. ESAIM: Control, Optimisation and Calculus of Variations, 20, pp 576-611. doi:10.1051/cocv/2013076, 2014.

[5] G. Allaire, F. Jouve, and G. Michailidis. Casting constraints in structural optimization via a level-set method. In WCSMO-10, Orlando, Florida, USA, 2013.

[6] G. Allaire, F. Jouve, and A.-M. Toader. A level-set method for shape optimization. C. R. Acad. Sci. Paris, Série I, 334:1125-1130, 2002.

[7] G. Allaire, F. Jouve, and A.-M. Toader. Structural optimization using sensitivity analysis and a level-set method. Journal of computational physics, 194(1):363-393, 2004.

[8] L. Ambrosio. Lecture notes on geometric evolution problems, distance function and viscosity solutions. Calculus of variations and partial differential equations. Topics on geometrical evolution problems and degree theory,G. Buttazo, A. Marino, M.K.V. Murthy Eds, pages 5-93, 1999.

[9] M.P. Bendsoe and O. Sigmund. Topology optimization: theory, methods and applications. Springer, 2004.

[10] S. Chen and W. Chen. A new level-set based approach to shape and topology optimization under geometric uncertainty. Structural and Multidisciplinary Optimization, 44(1):1-18, 2011.

[11] S. Chen, M.Y. Wang, and A.Q. Liu. Shape feature control in structural topology optimization. Computer-Aided Design, 40(9):951-962, 2008.

[12] G. Cheng, Y. Mei, and X. Wang. A feature-based structural topology optimization method. In IUTAM Symposium on Topological Design Optimization of Structures, Machines and Materials, pages 505-514. Springer, 2006.

[13] F. Clarke. Optimization and Nonsmooth Analysis. John Wiley \& Sons, New York, 1983.

[14] C. Dapogny. Optimisation de formes, méthode des lignes de niveaux sur maillages non structurés et évolution de maillages. PhD thesis, Université Pierre et Marie Curie-Paris VI, 2013, available at: http://tel.archives-ouvertes.fr/tel-00916224.

[15] F. de Gournay. Velocity extension for the level-set method and multiple eigenvalues in shape optimization. SIAM journal on control and optimization, 45(1):343-367, 2006.

[16] M.C. Delfour and J.-P. Zolésio. Shapes and geometries, volume 4 of Advances in Design and Control. Society for Industrial and Applied Mathematics (SIAM), Philadelphia, PA, 2001.

[17] M.C. Delfour and J.-P. Zolésio. Shape identification via metrics constructed from the oriented distance function. Control and Cybernetics, 34(1):137, 2005.

[18] L.C. Evans and R.F. Gariepy. Measure theory and fine properties of functions. Studies in Advanced Mathematics. CRC Press, Boca Raton, FL, 1992.

[19] J.K. Guest. Imposing maximum length scale in topology optimization. Structural and Multidisciplinary Optimization, 37(5):463-473, 2009.

[20] J.K. Guest. Topology optimization with multiple phase projection. Computer Methods in Applied Mechanics and Engineering, 199(1):123-135, 2009. 
[21] J.K. Guest. Optimizing the layout of discrete objects in structures and materials: A projection-based topology optimization approach. Computer Methods in Applied Mechanics and Engineering, 2014.

[22] J.K. Guest, A. Asadpoure, and S.-H. Ha. Eliminating beta-continuation from heaviside projection and density filter algorithms. Structural and Multidisciplinary Optimization, 44(4):443-453, 2011.

[23] J.K. Guest, J.H. Prévost, and T. Belytschko. Achieving minimum length scale in topology optimization using nodal design variables and projection functions. International Journal for Numerical Methods in Engineering, 61(2):238-254, 2004.

[24] X. Guo, W. Zhang, and W. Zhong. Explicit feature control in structural topology optimization via level set method. Computer Methods in Applied Mechanics and Engineering, 272(0):354 - 378, 2014.

[25] M. Hintermüller and W. Ring. A second order shape optimization approach for image segmentation. SIAM Journal on Applied Mathematics, 64(2):442-467, 2004.

[26] R. Kimmel and A.M. Bruckstein. Shape offsets via level sets. Computer-Aided Design, 25(3):154-162, 1993.

[27] R. Kimmel, D. Shaked, N. Kiryati, and A.M. Bruckstein. Skeletonization via distance maps and level sets. In Photonics for Industrial Applications, pages 137-148. International Society for Optics and Photonics, 1995.

[28] J. Luo, Z. Luo, S. Chen, L. Tong, and M.Y. Wang. A new level set method for systematic design of hinge-free compliant mechanisms. Computer Methods in Applied Mechanics and Engineering, 198(2):318-331, 2008.

[29] G. Michailidis. Manufacturing Constraints and Multi-Phase Shape and Topology Optimization via a Level-Set Method. PhD thesis, Ecole Polytechnique X, 2014, available at: http://pastel.archivesouvertes.fr/pastel-00937306.

[30] M. Minoux. Programmation mathématique: théorie et algorithmes, volume 1. Dunod Paris, 1983.

[31] U. Montanari. A method for obtaining skeletons using a quasi-euclidean distance. Journal of the $A C M$ (JACM), 15(4):600-624, 1968.

[32] J. Nocedal and S.J. Wright. Numerical optimization. Springer Series in Operations Research and Financial Engineering. Springer, New York, second edition, 2006.

[33] S.J. Osher and R. Fedkiw. Level set methods and dynamic implicit surfaces, volume 153 of Applied Mathematical Sciences. Springer-Verlag, New York, 2003.

[34] S.J. Osher and F. Santosa. Level set methods for optimization problems involving geometry and constraints: I. frequencies of a two-density inhomogeneous drum. Journal of Computational Physics, 171(1):272-288, 2001.

[35] S.J. Osher and J.A. Sethian. Fronts propagating with curvature-dependent speed: algorithms based on hamilton-jacobi formulations. Journal of computational physics, 79(1):12-49, 1988.

[36] J. Petersson and O. Sigmund. Slope constrained topology optimization. International Journal for Numerical Methods in Engineering, 41(8):1417-1434, 1998.

[37] O. Pironneau. Optimal shape design for elliptic systems. Springer Series in Computational Physics. Springer-Verlag, New York, 1984.

[38] T.A. Poulsen. A new scheme for imposing a minimum length scale in topology optimization. International Journal for Numerical Methods in Engineering, 57(6):741-760, 2003.

[39] J.A. Sethian. Level set methods and fast marching methods: evolving interfaces in computational geometry, fluid mechanics, computer vision, and materials science. Cambridge university press, 1999.

[40] J.A. Sethian and A. Wiegmann. Structural boundary design via level set and immersed interface methods. Journal of computational physics, 163(2):489-528, 2000. 
[41] O. Sigmund. On the design of compliant mechanisms using topology optimization. Journal of Structural Mechanics, 25(4):493-524, 1997.

[42] O. Sigmund. Morphology-based black and white filters for topology optimization. Structural and Multidisciplinary Optimization, 33(4-5):401-424, 2007.

[43] O. Sigmund. Manufacturing tolerant topology optimization. Acta Mechanica Sinica, 25(2):227-239, 2009 .

[44] O. Sigmund and J. Petersson. Numerical instabilities in topology optimization: a survey on procedures dealing with checkerboards, mesh-dependencies and local minima. Structural optimization, 16(1):68-75, 1998.

[45] J. Simon and F. Murat. Sur le contrôle par un domaine géométrique. Publication 76015 du Laboratoire d'Analyse Numérique de l'Université Paris VI, (76015):222 pages, 1976.

[46] J. Sokolowski and A. Zochowski. On the topological derivative in shape optimization. SIAM J. Control Optim., 37(4):1251-1272, 1999.

[47] J. Sokołowski and J.-P. Zolésio. Introduction to shape optimization, volume 16 of Springer Series in Computational Mathematics. Springer-Verlag, Berlin, 1992. Shape sensitivity analysis.

[48] K. Svanberg. The method of moving asymptotes-a new method for structural optimization. International journal for numerical methods in engineering, 24(2):359-373, 1987.

[49] SYSTUS2014, SYSWELD2014, User's manual, SYSTUS International, 2014.

[50] X. Wang, M.Y. Wang, and D. Guo. Structural shape and topology optimization in a level-set-based framework of region representation. Structural and Multidisciplinary Optimization, 27(1):1-19, 2004.

[51] W. Zhang, W. Zhong, and X. Guo. An explicit length scale control approach in simp-based topology optimization. Computer Methods in Applied Mechanics and Engineering, 2014.

[52] H. Zhao. A fast sweeping method for eikonal equations. Mathematics of computation, 74(250):603627, 2005. 